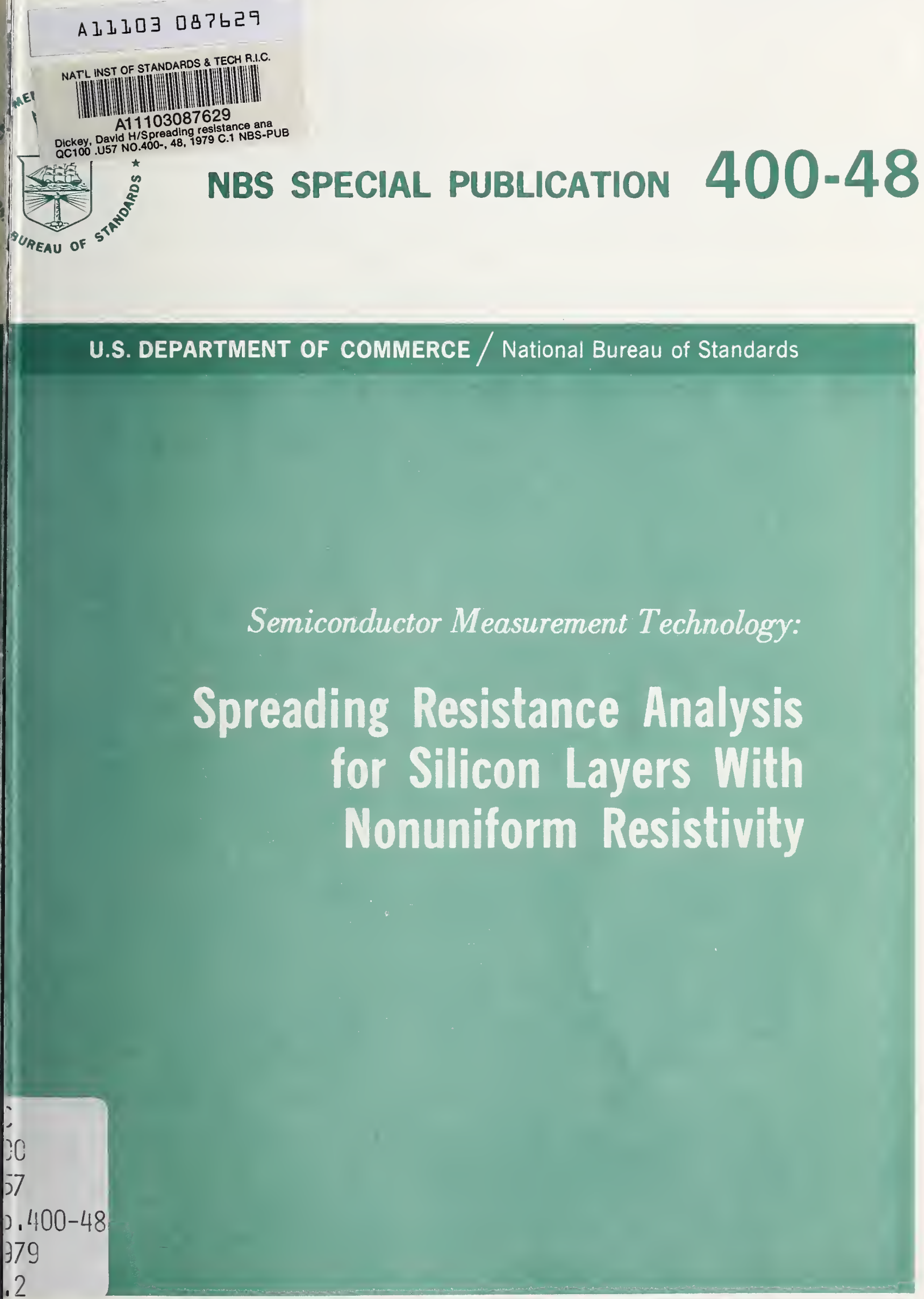


The National Bureau of Standards' was established by an act of Congress March 3, 1901. The Bureau's overall goal is to strengthen and advance the Nation's science and technology and facilitate their effective application for public benefit. To this end, the Bureau conducts research and provides: (1) a basis for the Nation's physical measurement system, (2) scientific and technological services for industry and government, (3) a technical basis for equity in trade, and (4) technical services to promote public safety. The Bureau's technical work is performed by the National Measurement Laboratory, the National Engineering Laboratory, and the Institute for Computer Sciences and Technology.

THE NATIONAL MEASUREMENT LABORATORY provides the national system of physical and chemical and materials measurement; coordinates the system with measurement systems of other nations and furnishes essential services leading to accurate and uniform physical and chemical measurement throughout the Nation's scientific community, industry, and commerce; conducts materials research leading to improved methods of measurement, standards, and data on the properties of materials needed by industry, commerce, educational institutions, and Government; provides advisory and research services to other Government Agencies; develops, produces, and distributes Standard Reference Materials; and provides calibration services. The Laboratory consists of the following centers:

Absolute Physical Quantities² - Radiation Research - Thermodynamics and Molecular Science - Analytical Chemistry - Materials Science.

THE NATIONAL ENGINEERING LABORATORY provides technology and technical services to users in the public and private sectors to address national needs and to solve national problems in the public interest; conducts research in engineering and applied science in support of objectives in these efforts; builds and maintains competence in the necessary disciplines required to carry out this research and technical service; develops engineering data and measurement capabilities; provides engineering measurement traceability services; develops test methods and proposes engineering standards and code changes; develops and proposes new engineering practices; and develops and improves mechanisms to transfer results of its research to the utlimate user. The Laboratory consists of the following centers:

Applied Mathematics - Electronics and Electrical Engineering ${ }^{2}$ - Mechanical

Engineering and Process Technology ${ }^{2}$ - Building Technology - Fire Research Consumer Product Technology - Field Methods.

THE INSTITUTE FOR COMPUTER SCIENCES AND TECHNOLOGY conducts research and provides scientific and technical services to aid Federal Agencies in the selection, acquisition, application, and use of computer technology to improve effectiveness and economy in Government operations in accordance with Public Law 89-306 (40 U.S.C. 759), relevant Executive Orders, and other directives; carries out this mission by managing the Federal Information Processing Standards Program, developing Federal ADP standards guidelines, and managing Federal participation in ADP voluntary standardization activities; provides scientific and technological advisory services and assistance to Federal Agencies; and provides the technical foundation for computer-related policies of the Federal Government. The Institute consists of the following divisions:

Systems and Software - Computer Systems Engineering - Information Technology.

Headquarters and Laboratories at Gaithersburg, Maryland, unless otherwise noted; mailing address Washington,D.C. 20234.

'Some divisions within the center are located at Boulder, Colorado, 80303.

The National Bureau of Standards was reorganized, effective April 9, 1978. 
Semiconductor Measurement Technology:

\section{Spreading Resistance Analysis for Silicon Layers With Nonuniform Resistivity}

\section{David H. Dickey}

Solecon Laboratories 3001 Red Hill Avenue

Costa Mesa, CA 92626

and

James R. Ehrstein

Electron Devices Division

Center for Electronics \& Electrical Engineering

National Engineering Laboratory

National Bureau of Standards

Washington D.C. 20234

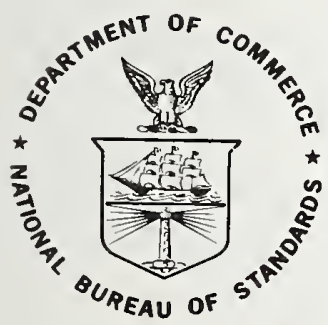

U.S. DEPARTMENT OF COMMERCE, Juanita M. Kreps, Secretary Jordan J. Baruch, Assistant Secretary for Science and Technology NATIONAL BUREAU OF STANDARDS, Ernest Ambler, Director 


\section{Library of Congress Cataloging in Publication Data}

Dickey, David H.

Semiconductor measurement technology.

(National Bureau of Standards special publication ; 400-48)

"This activity was supported by the Defense Advanced Research Projects Agency."

Supt. of Docs. no.: C13.10:400-48.

1. Semiconductors-Testing. 2. Electric resistance, Spreading. 3. Silicon-Electric properties. I. Ehrstein, James R., joint author. II. Title. III. Series: United States. National Bureau of Standards. Special publication ; 400-48.

QC100.U57 no. 400-48 [TK7871.85] 602'.1s [621.381 5'2'028] 79-607012

\section{National Bureau of Standards Special Publication 400-48}

Nat. Bur. Stand. (U.S.), Spec. Publ. 400-48, 72 pages (May 1979) CODEN: XNBSAV 
Abstract

1. Introduction

2. Theory .

2.1 The Boundary Value Problem

2.2 An Isolated Layer - Parallel Superposition of Conducting Sublayers

2.3 Layer over Conducting Substrate - Series Superposition of Sublayer Resistances . . . . . . . . . . . .

2.4 The Logarithmic Transformation . . . . . . . . . . 10

2.5 Local Slope . . . . . . . . . . . . . . . . . . . . . 12

3. Experiment . . . . . . . . . . . . . . . . . . 15

3.1 The Probe Spacing Experiment . . . . . . . . . . . 15

3.2 Diffusion Profiles . . . . . . . . . . . . . 18

3.3 Bevel-Edge Proximity Effect............... . . 32

4. Conclusions . . . . . . . . . . . . . . . . . . 32

References . . . . . . . . . . . . . . . . . . . 34

Appendix A . . . . . . . . . . . . . . . . . . . . 35

Appendix B . . . . . . . . . . . . . . . . . . . . . 51

Appendix C . . . . . . . . . . . . . . . . . . . . 57

Appendix D . . . . . . . . . . . . . . . . . . . 59

LIST OF FIGURES

1. Reciprocal of sampling volume correction factor for an isolated uniform slab plotted vs. normalized slab thickness . . . . . . . . . . . . . . . . . . . . .

2. Multilayer model appropriate for isolated slab, showing contacts extending through total slab thickness at the surface - parallel superposition, and at the new surface after removal of layer thickness $\Delta t$ - parallel superposition

3. Multilayer model for slab over conducting substrate - series

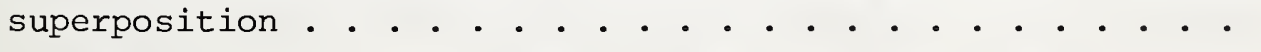

4. Schematic dependence of conductance and resistance on depth at which measurement is made below original surface of the structure

5. Schematic representation of slope-dependent terms for the correction factor $\mathrm{F}$, as derived from limiting case analysis.

6. Spreading resistance vs. probe spacing for junctionisolated layers 
7. Conductivity profile for boron diffusion into nominal $10-\Omega \cdot \mathrm{cm} n$-type substrate. Nominal surface density of boron, $10^{19} \mathrm{~cm}^{-3}$; junction depth by lap and stain, $0.7 \mu \mathrm{m}$.

8. Conductivity profile for boron diffusion into nominal 10- $\Omega \cdot \mathrm{cm} n$-type substrate. Nominal surface density of boron, $10^{19} \mathrm{~cm}^{-3}$; junction depth by lap and stain, $1.6 \mu \mathrm{m}$.

9. Conductivity profile for boron diffusion into nominal $10-\Omega \cdot \mathrm{cm} n$-type substrate. Nominal surface density of boron, $10^{19} \mathrm{~cm}^{-3}$; junction depth by lap and stain, $4.3 \mu \mathrm{m}$. .

10. Conductivity profile for boron diffusion into nominal 150- $\Omega \cdot \mathrm{cm} n$-type substrate . . . . . . . . . . . .

11. Conductivity profile for boron diffusion into nominal $1.0-\Omega \cdot \mathrm{cm} n$-type substrate . . . . . . . . . . . .

12. Conductivity profile for boron diffusion into nominal $0.03-\Omega \cdot \mathrm{cm} n$-type substrate

13. Conductivity profile for boron diffusion into nominal 100- $\Omega \cdot \mathrm{cm} n$-type substrate . . . . . . . . . . . . .

14. Conductivity profile for phosphorus diffusion into nominal $100-\Omega \cdot \mathrm{cm}$ p-type substrate

15. Conductivity profile for phosphorus diffusion into nominal 1.0- $\Omega \cdot \mathrm{cm}$ p-type substrate . . . . . . . . . . . .

16. Conductivity profile for phosphorus diffusion into nominal $0.03-\Omega \cdot \mathrm{cm}$ p-type substrate

17. Conductivity profile for phosphorus diffusion into nominal 150- $\Omega \cdot \mathrm{cm} n$-type substrate . . . . . . . . . . . .

18. Spreading resistance in the vicinity of the bevel edge

Al. Schematic conductivity profile for Computer Exercise I

A2. Radial potential distribution on a spreading resistance contact

A3. Schematic conductivity profile for Computer Exercises II and III.

Cl. Image array for infinite homogeneous wedge

C2. Sampling volume correction factor for uniform wedge with local thickness equal to contact radius . . . . . . . . .

C3. Sampling volume correction factor for uniform wedge with local thickness equal to 0.1 contact radii . . . . . . . .

Dl. Spreading resistance data for junction-isolated implanted specimen illustrating the type of local slope behavior near the top surface which gives nonphysical values for the correction factor when analyzed by eq $(22 \mathrm{a})$ or $(22 \mathrm{~b})$. . . . . 
1. Comparison of Diffused Layer Sheet Resistance Values for Three Boron Diffusions . . . . . . . . . . . . . . . .

2. Comparison of Diffused Layer Sheet Resistance Values for One $n$-Type Diffusion and One p-Type Diffusion . . . . . . . .

Al. Reciprocal Correction, $\mathrm{H}$, for a Single Layer of Thickness t on Lower-Conductivity, Infinite Substrate . . . . . . . . . 39

A2. Sampling Volume Correction Factors for Exercise II . . . . . 42

A3. Sampling Volume Correction Factors for Exercise III . . . . . 43

A4. The Ratio of Correction Factor from Parallel Superposition to that from Schumann and Gardner for Exercise II . . . . . .

A5. The Ratio of Correction Factor from Parallel Superposition to that from Schumann and Gardner for Exercise III . . . . . .

A6. The Ratio of Correction Factor from the Local Slope Method to that from Schumann and Gardner for Exercise II . . . . . .

A7. The Ratio of Correction Factor from the Local Slope Method to that from Schumann and Gardner for Exercise III . . . . . .

A8. Reciprocal Correction Factors for a Single Isolated Layer

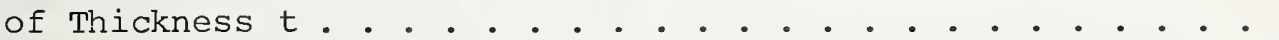

D. Correction Factor $F$ Based on Local Slope Using $K_{3}$ for Various Values of $R_{0} / R_{S}$ and $A l s o$ Using $K_{1} K_{2}$ and Unity to Replace $K_{3}$.

Dl. Case of Local Slope, $\mathrm{m}=-0.5$. . . . . . . . . . . 61

D2. Case of Local Slope, $\mathrm{m}=-0.1$. . . . . . . . . . . 61

D3. Case of Local Slope, $\mathrm{m}=0$. . . . . . . . . . . . . 62

D4. Case of Local Slope, $\mathrm{m}=0.1$. . . . . . . . . . . . 62

D5. Case of Local Slope, $\mathrm{m}=0.5$. . . . . . . . . . . . 63 
This study was carried out in part at Solecon Laboratories and in par at the National Bureau of Standards as part of the Semiconductor Technology Program in the Electron Devices Division. The Semiconductor Technology Program serves to focus NBS research to enhance the performance, interchangeability, and reliability of discrete semiconductor devices and integrated circuits through improvements in measurement technology for use in specifying materials and devices in national and international commerce and for use by industry in controlling device fabrication processes. This entire study was funded by the Defense Advanced Research Projects Agency through ARPA Order 2397. The work at Solecon Laboratories was funded through NBS Contract No. 5-35881. This contract was monitored by R. L. Raybold as the contracting officer's Technical Representative (COTR). Drs. W. M. Bullis and J. R. Ehrstein provided technical review of this contract for the National Bureau of Standards.

Certain commercial equipment, instruments, or materials are identified in this report in order to adequately specify the experimental procedure. In no case does such identification imply recommendation or endorsement by the National Bureau of Standards, nor does it imply that the material or equipment identified is necessarily the best available for the purpose. 


\section{Semiconductor Measurement Technology: \\ Spreading Resistance Analysis for Silicon Layers \\ with Nonuniform Resistivity}

by

David H. Dickey and James R. Ehrstein

\section{Abstract}

A simple mathematical algorithm is developed for the calculation of resistivity depth profiles from spreading resistance measurements on sectioned silicon device structures. It is applicable to structures consisting of one or more layers of the same or differing conductivity types. The algorithm accounts for modification of the sampling volume of the spreading resistance probes arising from nearby variations, in depth, of specimen resistivity whether resulting from graded dopant distribution or electrical boundaries, either insulating or conducting.

The algorithm is based on limiting case one- and twodimensional models of the conduction between the probes. Compared with the traditional spreading resistance analysis algorithm based on the threedimensional boundary value solution of Schumann and Gardner, the present work offers greatly reduced execution times even with a microcomputer, making real time analysis for process control possible.

An experiment to test the accuracy of one of the limiting models used is described. Profiles of several diffused layers generated from spreading resistance measurements analyzed by this algorithm are compared with profiles obtained on replicate specimens using another electrical technique. In addition, computer experiments are used for simple layer models to compare results based on this algorithm with results from the Schumann-Gardner approach.

Key Words: Dopant profiles; resistivity; resistivity profiles; semiconductor; silicon; spreading resistance.

\section{INTRODUCTION}

The spreading resistance technique is a powerful method for determining dopant distributions in silicon device structures. Much of the usefulness of the technique can be attributed to the relatively small sampling volume involved. However, modern silicon devices commonly have dimensions within their structure which are smaller than even this sam- 
pling volume, so it is often necessary to make calculations which remove the effect of specimen boundaries or large resistivity gradients from the measurements. Such calculations can be made only approximately and generally rely on various simplifying assumptions about the structure being considered. Of the approaches used for these calculations, that given by Schumann and Gardner (SG) [1] is most generally applicable because it is based on an approximate solution to the full threedimensional boundary value problem. Although several schemes [2-5] have been presented to reduce the complexity and computation time of the calculations associated with the SG approach, computer size and time required are still factors which inhibit its widespread usage.

In cases where the structure can reasonably be modeled in one or two dimensions, other approaches allowing vastly simpler calculations are possible. The work reported here is an investigation of the viability of some simpler approaches and has culminated in a scheme for making very rapid sampling volume corrections to spreading resistance measurements. The scheme is applicable to all commonly encountered structures and, in many cases, has an accuracy with reference to sG-based results which exceeds the reproducibility of the basic measurements. Briefly stated, the scheme uses differences between successive measurements on a profile as a basis for calculating sampling volume correction factors. During the course of the work, the theoretical relation between profile slope (difference in successive measurements) and required correction factor was developed. For the general case, there is not a one-to-one correspondence between slope and correction factor; but, for limiting cases, the relation can become exact. Computer exercises have been performed to test the "accuracy" of the theoretical results as defined by comparison with the SG approach. Laboratory experiments have been performed to test the slope-based scheme by comparing corrected spreading resistance profiles with profiles as measured by the technique of anodic oxidation-incremental sheet resistance. The limitingcase theoretical results have been combined in an empirical relation which in turn has been incorporated into a BASIC computer program for use in on-line data reduction.

The scheme presented here has a shortcoming which is shared by all other known methods: it does not account for bevel-angle and bevel-edge proximity effects [6]. These effects are sometimes important, yet virtually impossible to deal with theoretically. This report describes one brief theoretical consideration of the effect of the bevel angle and also includes the results of an experimental investigation of the beveledge proximity effect. Together, these results provide an estimate of the possible errors one makes by ignoring the effects.

\section{THEORY}

\subsection{The Boundary Value Problem}

The physical model assumed for a two-probe spreading resistance experiment consists of two circular equipotential regions on one surface 
of an infinite slab of resistive medium. The disks have radius, $a$; their centers are separated by a distance, $s$; and they are held at equal but opposite potentials, $+V$ and $-V$. The problem is to find the current between the disks. The most general case considered is that of a resistive slab having a variation in resistivity but only in the direction normal to its surface. The slab may have infinite thickness, or it may be bounded below by a layer of either infinite or zero resistivity.

For a slab of uniform resistivity and infinite thickness, the resistance $R$ between the disks is given in terms of measured voltage, $V$, between the disks, and current, I, by the well-known relation [7]:

$$
R=2 \mathrm{~V} / \mathrm{I}=\rho / 2 \mathrm{a},
$$

where $\rho$ is the electrical resistivity of the slab. If the slab has finite thickness, eq (1) must be corrected by a factor, F, which depends on thickness and contact separation, so that the resistance is given by

$$
\mathrm{R}=\rho \mathrm{F} / 2 \mathrm{a}
$$

In the following sections, discussion will be in terms of conductance as well as resistance, so it is convenient at this point to write the equivalent of eq (2) for the conductance between the disks:

$$
\mathrm{G}=2 \mathrm{a} \sigma \mathrm{H}=2 \mathrm{a} \sigma / \mathrm{F},
$$

where $\sigma$ is the (uniform) conductivity of the slab and $H$ is the reciprocal of the factor $F$.

Exact calculation of the factor $F$ (or its reciprocal) for a uniform slab requires the solution of a potential-theory problem having mixed boundary conditions. Moreover, it is a two-center problem; but this aspect can be safely handled by superimposing the potential distributions around each disk treated alone as long as their separation is a few times the disk radius. The mixed-boundary-condition aspect formally requires the solution of a pair of dual-integral equations which do not have a general analytical solution. With the assumption of a particular current distribution under each disk appropriate to a semi-infinite specimen, SG used a known solution to the dual-integral equations to evaluate $F$. Although their assumption is not generally valid, its use introduces errors which (see Appendix A, sec. A.4) do not exceed a few percent. It should be noted that even the most streamlined version [5] of their approach requires at least one numerical integration over a fine scale grid with an integrand involving several transcendental functions.

It is desirable to make certain simplifying assumptions in the model used in order to formulate a sampling volume correction scheme which is operationally simpler than that resulting from the boundary value solution of SG. 


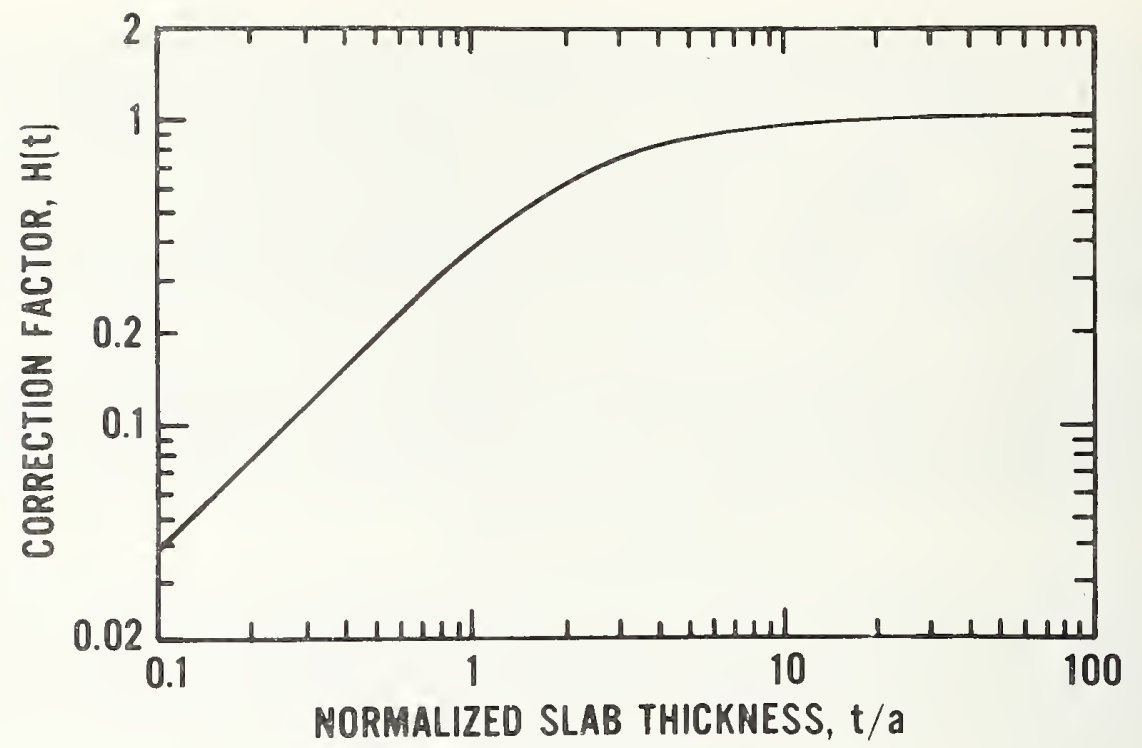

Figure 1. Reciprocal of sampling volume correction factor for an isolated uniform slab plotted vs. normalized slab thickness. Probe spacing equal to 40 contact radii.

The method of images (detailed in sec. A.2 of Appendix A) was used to find numerical values of $F$ for a two-layer structure to a very high accuracy. This is done for a more general current distribution under the contact than that assumed by SG, but one that is shown to come much closer to meeting their assumed boundary condition of constant potential under the contact. The image calculation results in the dependence of $\mathrm{H}$, the reciprocal of the correction factor $\mathrm{F}$, on slab thickness shown in figure 1 .

For a slab consisting of a large number of layers of differing resistivities, the method of images approach becomes very complicated. The solution can be simplified in cases which can be modeled in fewer than three dimensions.

In practice, there are a great number of structures in which the total effective thickness is less than the contact radius. In these cases, models can be constructed in which current is exclusively parallel to coordinate directions. The most common case is that of a shallow diffusion or implant where a $p-n$ junction isolates the material being probed from the substrate. In such cases, the current is two-dimensional and predominantly parallel to the surface of the layer. A useful model is one in which the equipotential contacts are cylinders extending through the thickness of the layer. This model is used in section 2.2 as a starting point to derive a more general solution according to the principle of parallel superposition. Another limiting two-dimensional current is approximated by a thin, relatively high resistivity structure on a low resistivity substrate. Although the substrate may be very thick, 
the dominant contribution to measured resistance in this case is in the high resistivity layers where current is predominantly normal to the surface. A simple solution involving differences in successive measurements is obtained from this model in section 2.3. Because the concepts to follow are developed in terms of removing physical layers, a process which normally proceeds from the top of the structure, a position coordinate system is defined whose origin is at the original specimen surface and which increases into the depth of the specimen's structure; see figures 2 and 3.

\subsection{An Isolated Layer - Parallel Superposition of Conducting Sublayers}

For a thin slab with uniform conductivity on an insulating substrate, the conductance between two circular contacts on the surface is given by eq (3), where $H$ depends on slab thickness $t$ as derived in Appendix A and shown in figure 1. For thickness less than the contact radius, $H(t)$ is always less than unity, see figure 1 , and has for an asymptotic limit at very small thicknesses [8] ${ }^{\dagger}$

$$
H(t)=\frac{\pi t}{2 a \ln (s / a)} \text {. }
$$

In this asymptotic region, the current is predominantly in the plane of the slab and the model is equivalent to one in which the contacts are cylinders extending throughout the thickness of the slab. If a thin slab having nonuniform resistivity with depth is now subdivided, as shown in figure $2 a$, into $N$ layers each with different conductivity, the conductance between the cylindrical contacts is given by

$$
G_{I}=\frac{\pi}{\ln (s / a)} \sum_{i=1}^{N} \sigma_{i} \Delta t,
$$

where $\Delta t$ is the common thickness of each sublayer. In keeping with the position coordinate convention above, the index, $i$, increases into the depth of the structure. Equation (5) is the extension of eq (3) using eq (4) for $H(t)$. To fit the assumption of independent layers conducting in parallel, one may consider the layers separated by virtual insulators. Therefore, $\mathrm{H}$ is evaluated for the thickness to the nearest insulator, namely, $\Delta t$. If the top sublayer is now removed, the conductance becomes

$$
\begin{aligned}
G_{2} & =\frac{\pi}{\ln (s / a)} \sum_{i=2}^{N} \sigma_{i} \Delta t \\
& =G_{1}-\frac{\pi}{\ln (s / a)} \sigma_{i} \Delta t .
\end{aligned}
$$

The result stated in the reference incorrectly gives $H(t)=\frac{\pi t}{2 \operatorname{an}(2 \mathrm{~s} / \mathrm{a})}$. 


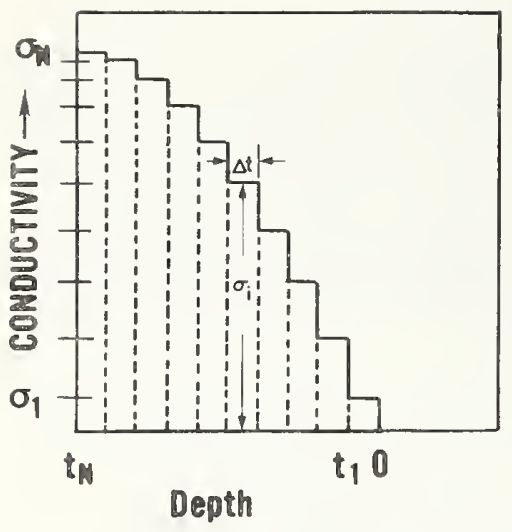

(a)

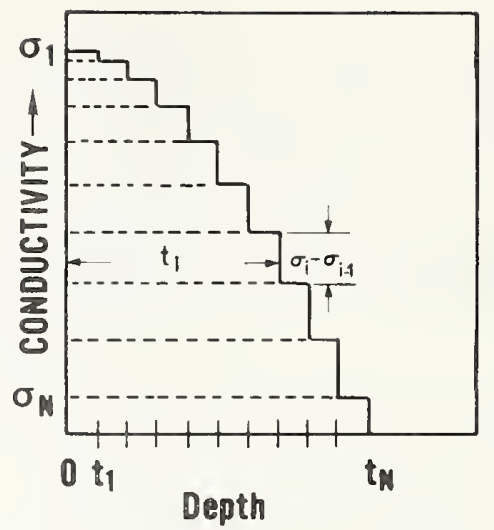

(b)

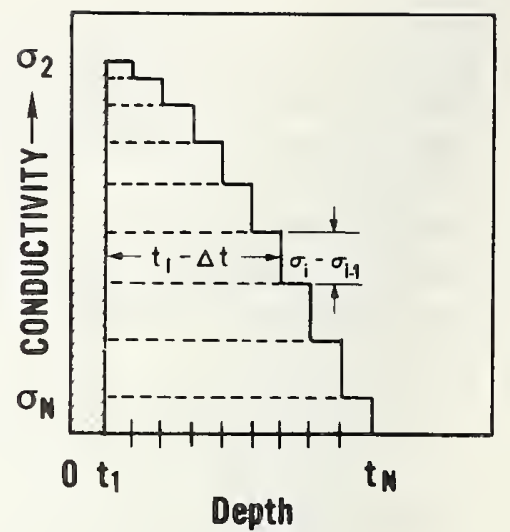

(c)

Figure 2. Multilayer model appropriate for isolated slab, showing contacts (a) extending through total slab thickness, (b) at the surface parallel superposition, and (c) at the new surface after removal of layer thickness $\Delta t$ - parallel superposition.

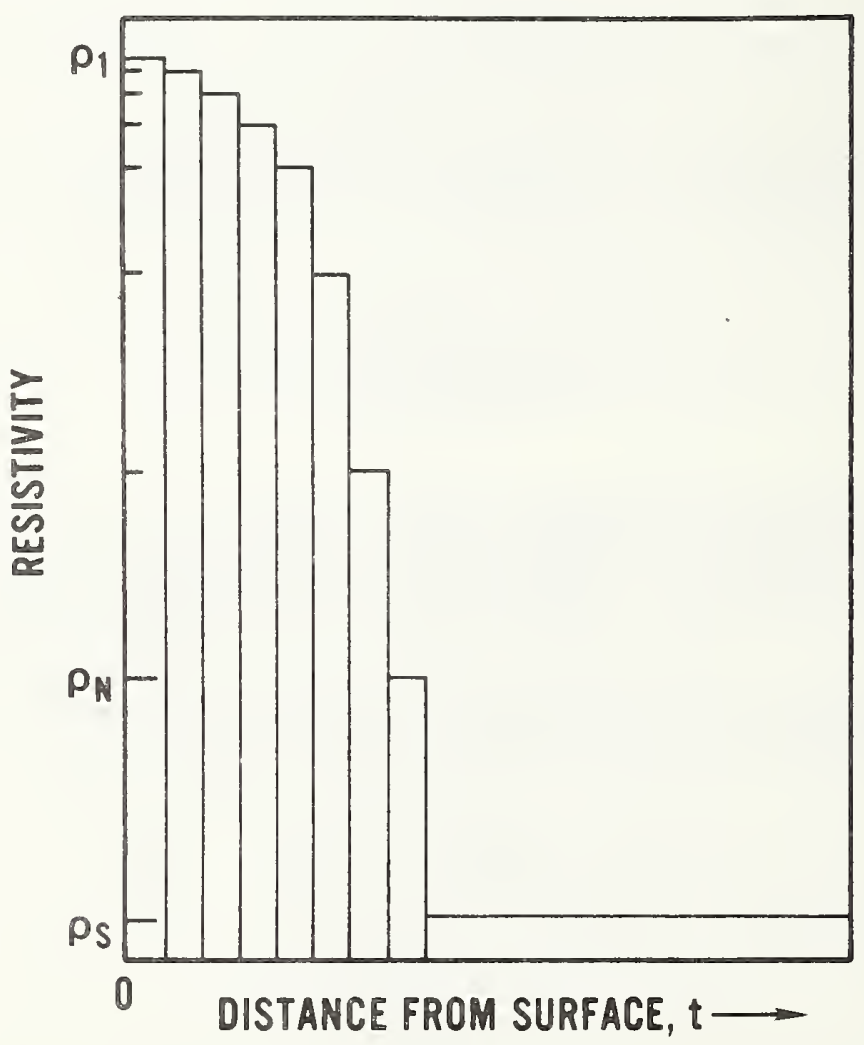

Figure 3. Multilayer model for slab over conducting substrate - series superposition. 
Rearranging eq (6b), one finds for this limiting case of small total thickness that the surface conductivity is

$$
\sigma_{1}=\frac{\ln (s / a)}{\pi}\left[\frac{G_{1}-G_{2}}{\Delta t}\right] \text {. }
$$

The conductivity of the next sublayer can be similarly found by removing that layer and measuring a third conductance. Thus, the entire slab can be profiled by successively removing each sublayer and using the difference in measured conductance before and after removal of the sublayer to calculate each conductivity according to the generalized form of eq (7). This equation is closely analogous to the relation used with a four-probe incremental sheet resistance experiment [9].

The preceding analysis assumed a thin slab so that eq (4) would be valid. For thicker slabs, where probes cannot be represented as extending through the structure, a similar analysis can be made by subdividing the slab not into sublayers of equal thickness but into layers of fractional conductivity and incrementally increasing thickness as depicted in figure $2 \mathrm{~b}$. As before, the layers are separated by virtual insulators to assure conduction in parallel. Although the layers act as resistors conducting in parallel, parts of the resistors happen to occupy the same physical space. This model is then a mathematical construction utilized to investigate the ramifications of extending the concept of parallel superposition beyond the limiting case of very thin layers. Writing the conduction of the slab as the sum of $\mathrm{N}$ individual conductances:

$$
G_{1}=2 a \sum_{i=1}^{N}\left[\sigma_{i}-\sigma_{i+1}\right] H\left(t_{i}\right)
$$

where $H\left(t_{j}\right)$ is the uniform-layer correction factor for thickness $t_{i}$. In this model, each layer extends to the surface so the assumption of $f^{i}$ contacts extending through the slab is no longer needed. If a physical layer of thickness $\Delta t$ is now removed from the top of the slab, the top layer $\left(t_{i}=\Delta t\right)$ of the mathematical representation disappears and all other laỵers become smaller by $\Delta t$; see figure $2 \mathrm{c}$. The new conductance is then

$$
G_{2}=2 a \sum_{i=2}^{N}\left[\sigma_{i}-\sigma_{i+1}\right] H\left(t_{i}-\Delta t\right),
$$

where $t_{i}$ is retained as the original thickness of the $i$ th layer. Since $t_{i}-\Delta t^{i}$ is just $t_{i-l^{\prime}}$ eqs (8) and (9) can be rewritten in equivalent form after shifting the indices on the terms involving $\sigma_{i+1}$ : 


$$
\begin{aligned}
& \frac{1}{2 a} G_{1}=\sigma_{1} H\left(t_{1}\right)+\sum_{i=2}^{N} \sigma_{i}\left[H\left(t_{i}\right)-H\left(t_{i-1}\right)\right]-\sigma_{N+1} H\left(t_{N}\right) \\
& \frac{1}{2 a} G_{2}=\sigma_{2} H\left(t_{0}\right)+\sum_{i=2}^{N} \sigma_{i}\left[H\left(t_{i-1}\right)-H\left(t_{i-2}\right)\right]-\sigma_{N+1} H\left(t_{N-1}\right) .
\end{aligned}
$$

The first term on the right in eq (11) is included for algebraic accuracy to balance an equal term included in the sum, although both terms are zero because $t_{0}$ is zero. Similarly, $\sigma_{N+1}$ in the model (fig. $2 b$ ) is zero, so these terms are dropped and the difference in conductance is taken as:

$$
\frac{1}{2 a}\left(G_{1}-G_{2}\right)=\sigma_{1} H\left(t_{1}\right)+\sum_{i=2}^{N} \sigma_{i}\left[H\left(t_{i}\right)-2 H\left(t_{i-1}\right)+H\left(t_{i-2}\right)\right] \text {. }
$$

If the thickness of the top layer, $t_{i}(=\Delta t)$ is small enough that $H\left(t_{1}\right)$ is in the asymptotic region, $\mathrm{H}\left(\mathrm{t}_{1}\right)$ can be replaced with its value as given by eq (4) and eq (12) can be rearranged to find $\sigma_{1}$ :

$$
\sigma_{1}=\frac{\ln (s / a)}{\pi} \frac{G_{1}-G_{2}}{\Delta t}-\frac{2 a \ln (s / a)}{\pi \Delta t} \sum_{i=2}^{N} \sigma_{i} \Delta^{2} H\left(t_{i-1}\right),
$$

where the symbol $\Delta^{2} \mathrm{H}$ denotes the second difference appearing in the square brackets in eq (12) [10].

This second difference is always negative (note from fig. 1 that $H(c)$ is concave downward for the case of insulating substrate), but it approaches zero for either very small or very large values of $t$. Equations (7) and (13) are thus seen to agree for the case of small total slab thickness. The second term in eq (13) may be regarded as a correction to account for the contribution from subsurface layers in a thick slab. To use this formula for a thick slab, one must first apply it to the deepest points on a profile, and then work out toward the surface. For more detail see Appendix B. When used in this way, the formula gives the correct result (at least for points far from the insulating substrate) even for a very thick slab of uniform conductivity. In this case, the first term is negligible and the second can be shown to sum to $\sigma$ since the $\sigma$ 's are constant and can be taken outside the summation. The exact form of $\mathrm{H}(t)$ has not been specified at this point because it is not critical: $H(t)$ needs only to asymptotically approach unity for large thickness and the value given by eq (4) for small $t$.

The analysis leading to eq (13) is based on the concept called Parallel superposition: that the conductance of a nonuniform slab can be written as a sum of parallel conductances. This model was expected 
to work well for thin layers with insulating substrates; but at the time this work was begun, it was not known whether the concept would yield exact results for intermediate cases of thicker slabs having a wide variation in conductivity. A computer experiment was performed to test the concept for a few of these intermediate cases with the conclusion that it is not exact for the general case. The results of the computer experiments are described in Appendix A.

\subsection{Layer over Conducting Substrate - Series Superposition of Sub- Layer Resistances}

For a thin, high resistivity structure on a low resistivity substrate, most of the resistance measured between two disk contacts on the surface arises in the high resistivity layers. In the limiting case where the total thickness of this region is much less than the contact diameter, the current through the region is predominantly normal to the surface of the structure. Figure 3 is an illustration of such a structure. The resistance measured at the surface in this case can be written, approximately, as the sum of the incremental resistances in the cylinders below each contact disk and the spreading resistance between virtual contacts on the substrate:

$$
R_{1}=2 \sum_{i=1}^{N} \rho_{i} \frac{\left(t_{i}-t_{i-1}\right)}{\pi a^{2}}+\frac{\rho_{s}}{2 a}
$$

where $\rho_{\mathrm{s}}$ is the resistivity of the substrate. This formula would be a closer approximation if the radii used in each term in the sum were progressively larger to account for slight spreading with depth, but the equation is quite exact for the limiting case of small t 's and large values of $\rho / \rho_{i}$. If the layer nearest the surface is removed, the new resistance $\mathbf{w i l} \underline{I}$ be smaller by just the first term in the sum, and the resistivity at the original surface can be found from

$$
\rho_{1}=\frac{\pi a^{2}}{2}\left[\frac{R_{1}-R_{2}}{\Delta t}\right]=\frac{\pi a^{2}}{2}\left[\frac{\Delta R}{\Delta t}\right]
$$

where $R_{2}$ is the measured resistance after removal of the layer and $\Delta t$ is tire (positive) thickness of the removed layer.

The important conclusion reached from this limiting case analysis is that the surface resistivity can be found from the difference in successive measurements, just as it was for the case of current parallel to the surface. In the parallel case, the measurements were in terms of conductance, and in the present case resistance measurements are made. In actual practice with common spreading resistance electronics, measurements are made in terms of the logarithms of resistance. In the following section a convenient transformation is described which puts both eqs (13) and (15) in terms of logarithms of measured resistance. 


\subsection{The Logarithmic Transformation}

The basic spreading resistance equation for a two-probe experiment is given in eq (2), if $\mathrm{F}$ in that equation is the sampling volume correction factor. Formulas for $F$ can be extracted from eqs (13) and (15) since they both contain factors of the type $\Delta Y / \Delta t$. If $\Delta Y$ and $\Delta t$ are both small so that the differential limit may be assumed, these factors can be replaced as follows:

$$
\Delta \mathrm{Y} / \Delta t \rightarrow \mathrm{Y} \Delta \ln (\mathrm{Y}) / \Delta t
$$

With this substitution, eq (13) and eq (15) become:

$$
\frac{\sigma}{G}=\frac{\ln (s / a)}{\pi}\left[\frac{\Delta \ln G}{\Delta t}-\frac{2 a}{G \Delta t} \sum_{i=2}^{N} \sigma_{i} \Delta^{2} H\left(t_{1-1}\right)\right]
$$

and

$$
\frac{\mathrm{R}}{\rho}=\frac{2}{\pi \mathrm{a}^{2}} \cdot \frac{\Delta t}{\Delta \ln R}
$$

The following is then extracted from eq (3) and eq (13a):

$$
F=\frac{2 a \ln (s / a)}{\pi}\left[\frac{\Delta \ln G}{\Delta t}-\frac{2 a}{G \Delta t} \sum_{i=2}^{N} \sigma_{i} \Delta^{2} H\left(t_{i-1}\right)\right]
$$

and from eq (2) and eq (15a):

$$
F=\frac{4 \Delta t}{\pi a \Delta \ln R}
$$

Further substitutions in eq (16) are made, In $G=-$ in $R$ and $G=$ $1 / R$, to make eq (16) comparable in form with eq (17), i.e., based on the directly measured quantity, log of resistance. This has the effect of formally changing the sign of the first term in eq (16) and then canceling the effect of this change, since for the same data $\frac{\Delta \mathrm{ln} G}{\Delta t}$ and $\frac{\Delta \ln R}{\Delta t}$ have opposite signs:

$$
F=\frac{-2 a \ln (s / a)}{\pi}\left[\frac{\Delta \ln R}{\Delta t}-\frac{2 a R}{\Delta t} \sum_{i=2}^{N} \sigma_{i} \Delta^{2} H\left(t_{i-1}\right)\right] \text {. }
$$

In practice, it is preferable to establish the relationship between spreading resistance and resistivity with a calibration curve rather than with a calculation based on eqs (2). (13), or (15). An appealing feature of the logarithmic transformation is that it preserves the linear relation between resistance and resistivity so that the cali- 
bration curve can still be used. Equations (16a) or (17) can be used to find the correction factor, and resistivity can then be found from the calibration curve using the corrected resistance, $R / F$. Note that without the logarithmic transform, eqs (13) and (15) relate resistivity to terms such as $\Delta \mathrm{R} / \Delta \mathrm{t}$ which are not necessarily linear in $\mathrm{R}$.

Converting the results of the initial derivation to a form compatible with data as measured requires several transformations or changes of reference point, each entailing possible sign changes. Remembering that the correction factor, $F$, must be positive since it is the ratio of positive quantities $R, a$, and $\rho$, it is useful to consider the effect of sign convention on the component terms. Because of the definition of the coordinate, $t$, in the derivation of eqs (13) and (15), $\Delta t=t,-t i-1$ is positive although it corresponds to the removal of a physical tayer. The differences $\Delta G=G_{i-1}-G_{i}$ and $\Delta R=R_{i-1}-R_{i}$, as constructed, are also positive because for $^{-1}$ the ${ }^{i}$ physical situations described: those of parallel conductances and series resistances, respectively, the respective total conductance and resistance are larger for positions near the surface than for positions into the depth of the structures. The ratios, $\frac{\Delta G}{\Delta t}$ [from eq (16)] and $\frac{\Delta R}{\Delta t}$ [from eq (17)], are positive as defined for the the coordinate $t$ starting at the top surface; also, $\frac{\Delta R}{\Delta t}$ [from eq (16a)] is negative. It is to be noted, however, that $\Delta \mathrm{G}$ and $\Delta \mathrm{R}$ are defined in the opposite sense from $\Delta t$ for the interval being considered, for example, $\Delta G \equiv \frac{G_{\text {initial }}-G_{\text {final }}}{t_{\text {final }}-t_{\text {initial }}}$. As a result, the ratios, $\frac{\Delta G}{\Delta t}$ and $\frac{\Delta R}{\Delta t}$ are the negative of the ratios $\frac{\Delta G}{\Delta t}$ and $\frac{\Delta R_{m}}{\Delta t}$ taken from the slopes of the measured conductance, $G$ VS. $t$, and the measured resistance, $R$ vs. $t$, relations as they would be taken directly from acquired data, see figure 4 . The equations for $F$ are rewritten using $R$ to reflect the evaluation of the $\Delta R$ interval in the same sequence as the $\Delta t$ interval, the top surface still being taken as the origin:

$$
\begin{aligned}
& F=\frac{2 a \ln (s / a)}{\pi}\left[\frac{\Delta \ln R_{m}}{\Delta t}-\frac{2 a R_{m}}{\Delta t} \sum_{i=2}^{N} \sigma_{i} \Delta^{2} H\left(t_{i-1}\right)\right] \\
& F=\frac{-\Delta \Delta t}{\pi a \Delta \ln R_{m}} .
\end{aligned}
$$

For the parallel superposition model, illustrated in figure $4 a$, the first term in eq (16b) is positive; the second is the negative of a term, $\Delta^{2} \mathrm{H}$, itself negative, as discussed subsequent to eq (13). The value for $\mathrm{F}$ in eq (17a) appropriate to the series superposition model, is the negative reciprocal of the slope of the $R_{m}$ is. $t$ relation in figure $4 b$, itself negative. Hence, in both cases, $M_{F}$ is clearly positive as it must be, since it is the ratio, $2 a R / \rho$ of positive quantities. 

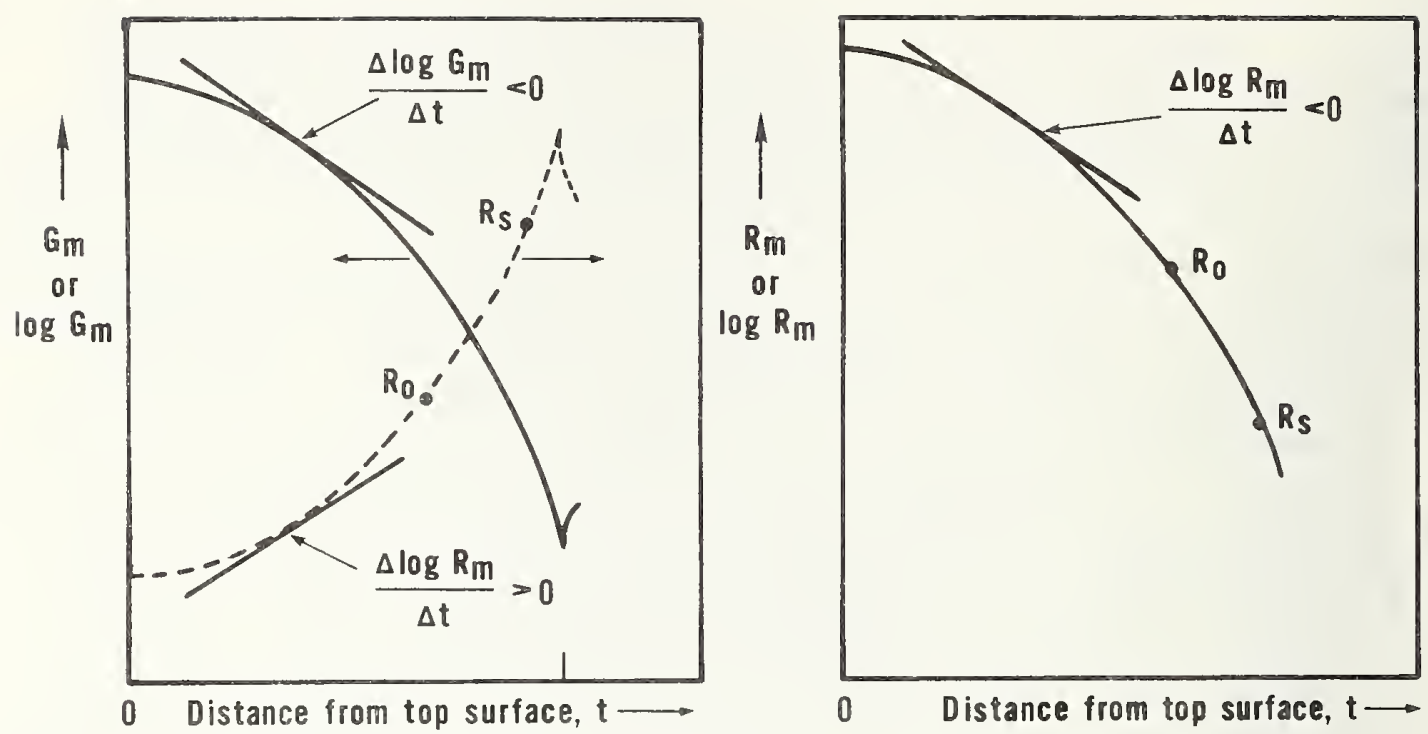

a. Case of isolated slab: parallel b. Case of conducting substrate superposition model. not insulated from slab: series superposition model.

Figure 4. Schematic dependence of conductance and resistance on depth at which measurement is made below original surface of the structure. Reverse coordinates, referenced to layer-substrate interface also shown. slopes, tangent to curves, as shown, are referred to coordinates originating at top surface. Schematic values of $R_{0}$ anc $R_{S}$ are shown for reference in interpreting the substrate factor $K_{3}$.

\subsection{Local Slope}

The equations developed in the preceding section provide limiting case descriptions of the behavior of the sampling volume correction factor. In both cases the behavior is governed by the slope of the spreading resistance data profile. The local slope of the measured resistance profile, $\Delta l n R / \Delta t$, is written as $m$, and the thin layer limiting forms for $F$ become: ${ }^{m}$

$$
F=K_{1} m, \text { for } m>0
$$

and

$$
\mathrm{F}=-\mathrm{K}_{2} / \mathrm{m} \text {, for } \mathrm{m} \ll 0
$$

where

$$
K_{1}=\frac{2 a \ln (s / a)}{\pi}
$$

and

$$
\mathrm{K}_{2}=\frac{4}{\pi \mathrm{a}} .
$$




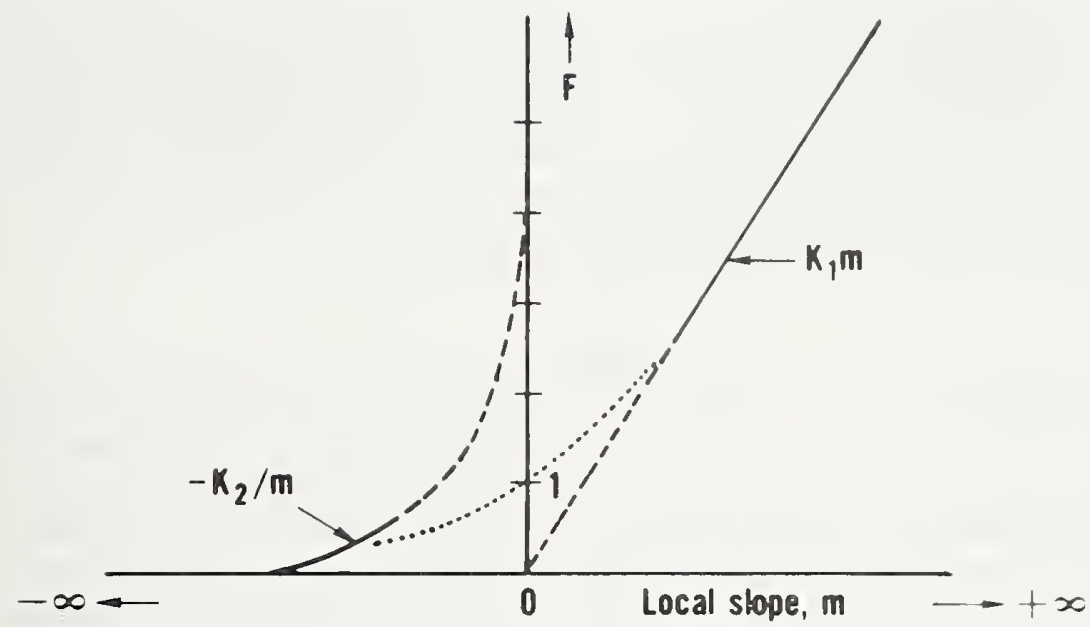

Figure 5. Schematic representation of slope-dependent terms for the correction factor $F$, as derived from limiting case analysis: solid lines regions where the terms are applicable; dashed lines - regions of questionable validity; dotted line - hypothesized form for $\mathrm{F}$ in region of intermediate slope.

It is to be noted that the natural unit for the local slope, $\mathrm{m}$, is decades (of resistance change) per micrometer of depth into the specimen.

In the asymptotic form for large positive slope, the second term in eq (16) is ignored because it is always negligible relative to the slope term under these conditions. Since the second difference of $\mathrm{H}(t)$ is always negative (or zero), this second term will always make a positive (or zero) contribution to $\mathrm{F}$.

For near zero slope, indicative of remote lower boundary, nearly uniform material, or both, $F$ should be on the order of unity and neither of the above forms is realistic. Figure 5 illustrates the asymptotic forms given above and the expected approximate form of $\mathrm{F}$ for intermediate values of $\mathrm{m}$. The two asymptotic forms above can be combined into a single hypothesized form which displays the correct asymptotic behavior for large positive and negative slope

$$
F=K_{1} m / 2+\sqrt{\left(K_{1} m / 2\right)^{2}+K_{1} K_{2}} .
$$

When the slope is zero, this equation reduces to a value near unity.

$$
F=\sqrt{K_{1} K_{2}}=\sqrt{8 \ln (s / a) / \pi^{2}} .
$$

Equation (22) is a reasonable approximation to the sampling volume correction factor for any profile, regardless of whether the slope is positive, zero, or negative. The error resulting from the formula is largest for profiles on bulk samples of uniform resistivity, for which 
the slope is zero and the sampling volume correction factor should be unity. For example, with a probe spacing, s, equal to 40 contact radii, $a$, the factor $\mathrm{K}_{1} \mathrm{~K}_{2}$ on a profile with zero slope is approximately 3.0 which results in a value for $F$ of about 1.7 , as opposed to unity. However, for either large positive or large negative slope, eq (22) rapidly approaches the correct limiting value.

An improvement can be made in the overall applicability of the local-slope formula in the range of intermediate $m$ values by replacing the constant $\mathrm{K}_{1} \mathrm{~K}_{2}$ in eq (22) by a variable factor which depends on the measured resistance approximately one contact radius deeper on the profile. This variable factor is called the substrate factor, $\mathrm{K}_{3}$. For the bulk specimen case mentioned above, the resistance one contact radius deeper is equal to the resistance at the point being corrected, so for this condition the variable factor $\mathrm{K}_{3}$ should have the value of unity. The formula tentatively adopted for the substrate factor is

$$
\mathrm{K}_{3}=1+\left(\mathrm{K}_{1} \mathrm{~K}_{2}-1\right)(2 / \pi) \tan ^{-1}\left[\log \left(\mathrm{R}_{\mathrm{o}} / \mathrm{R}_{\mathrm{S}}\right)\right],
$$

where $R$ and $R$ are the measured, $i . e .$, uncorrected, resistances at the point being corrected and at a point approximately one contact radius deeper on the profile, respectively; the arctan function is expressed in radians. This form, admittedly arbitrary, was chosen because it is a continuous, monotonic function in the interval $O<R / R<\infty$ and because it contributes to $F$ in the same sense that the $\Delta^{2} H$ term ${ }^{S}$ does in eq (16). This formula gives values for the substrate factor of

$$
\begin{aligned}
& \mathrm{K}_{3}=\mathrm{K}_{1} \mathrm{~K}_{2} \text { for } \mathrm{R}_{\mathrm{O}} / \mathrm{R}_{\mathrm{S}} \gg 1 \text { (conducting substrate, } \mathrm{m}<0 \text { ), } \\
& \mathrm{K}_{3}=1 \text { for } \mathrm{R}_{0} / \mathrm{R}_{\mathrm{S}}=1 \text { (bulk specimen, } \mathrm{m} \simeq 0 \text { ), and } \\
& \mathrm{K}_{3}=2-\mathrm{K}_{1} \mathrm{~K}_{2} \text { for } \mathrm{R}_{0} / \mathrm{R}_{\mathrm{S}} \ll 1 \text { (insulating substrate, } \mathrm{m}>0 \text { ). }
\end{aligned}
$$

The values for the first two conditions lead to accurate sampling volume correction factors, and since the value of $\mathrm{K}_{3}$ has negligible influence on the magnitude of $F$ in the case of large positive slope (the third condition above), acceptably accurate results are expected for all conditions if the term $\mathrm{K}_{1} \mathrm{~K}_{2}$ in eq (22) is replaced by $\mathrm{K}_{3}$. (See also the discussion in Appendix $B$ and Appendix $D$ for operational application to real data.)

The final operating equation for $F$ which is expected to have general application in depth profiling by spreading resistance is, therefore,

$$
F=\frac{K_{l} m}{2}+\sqrt{\left(\frac{K_{l} m}{2}\right)^{2}+K_{3}},
$$

assuming the data are analyzed from the near surface side of the layer working into its depth, as was done in the derivation. However, since the form $\mathrm{K}_{3}$ requires evaluation of the resistance at point below the one 
for which the factor $F$ is being evaluated, it becomes more convenient to proceed with the analysis from the bottom of the structure. For analysis starting at the deepest part of the layer and working towards the shallowest, as is done in Appendix B, the computed slope has the opposite sense from the foregoing, resulting in:

$$
F=-\frac{K_{1} m}{2}+\sqrt{\left(\frac{-K_{1} m}{2}\right)^{2}+K_{3}} .
$$

\section{EXPERIMENT}

\subsection{The Probe Spacing Experiment}

For the case of a uniform layer, thin enough that $H(t)$ is in the asymptotic region, on an insulating substrate, the resistance measured between the two contacts is [from eqs (2) and (4)]:

$$
R=\rho \ln (s / a) / \pi t=\frac{\rho}{\pi t}[\ln (s)-\ln (a)] .
$$

If $\ln (s)$ is the independent variable, this is an equation of the type

$$
y=m(x-b)
$$

where the slope, $m$, is $\rho / \pi t$ and the $x$-axis intercept, $b$, is $\ln (a)$. The experiment suggested by this equation is to measure spreading resistance on a thin layer at various values of probe spacing and to plot the results with $\mathrm{R}$ on a linear scale and $\mathrm{s}$ on a logarithmic scale. The plotted points should fall on a straight line with slope $2.3 \mathrm{\rho} / \pi \mathrm{t}$ (ohms per decade) and with an intercept on the horizontal axis $(R=0)$, at an extrapolated spacing equal to the contact radius. This experiment was performed with various layers and probe loadings, with consistent results. Typical results for three thin $p$-type diffused layers with different junction depths are shown in figure $6 a$ and results are summarized in table 1. The resistivity in these layers is not uniform, but if $\rho / t$ is interpreted in eq (25) as being the sheet resistance, close agreement is found between the slopes in figure $6 a$ and sheet resistances measured on the specimens by four-probe, see table 1. Note that in figure $6 a$ the same value of intercept is found for all three p-type layers, indicating a common value of the contact radius, a. Figure $6 \mathrm{~b}$ and table 2 show the results of a similax experiment performed on both, $n$ - and p-type diffusions with two probe loads. Note that the value of slope does not depend on probe load for either $p$ - or $n$-type specimens, and consistent values of sheet resistance are obtained. The difference in intercepts for $n$ - and $p$-type layers at a given load implies either a difference in contact radius for the two types of material or the presence of a type dependent series resistance. Values of $a$, derived from the x-axis intercept, $1 n(a)$, eq (25), in such probe spacing experiments, were used in calculating correction factors, $F$, used in profile analyses in section 3.2 .

The important result of these experiments is that the measured spreading resistance on an isolated, thin, nonuniform layer is indeed 


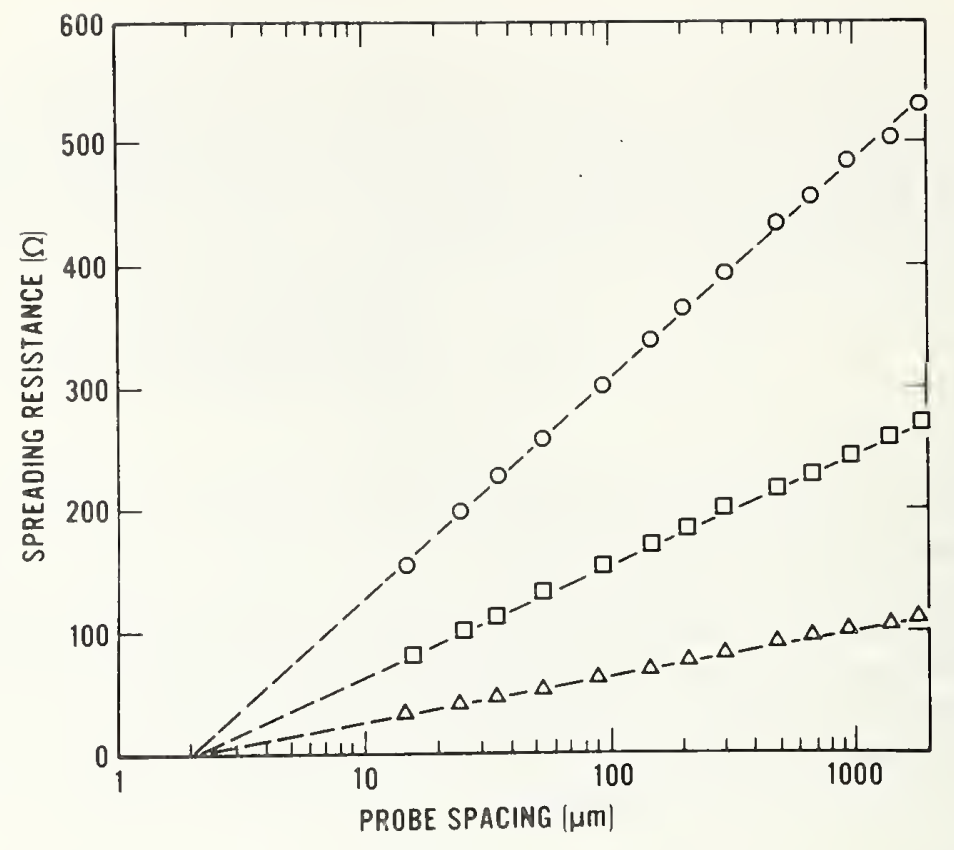

(a)

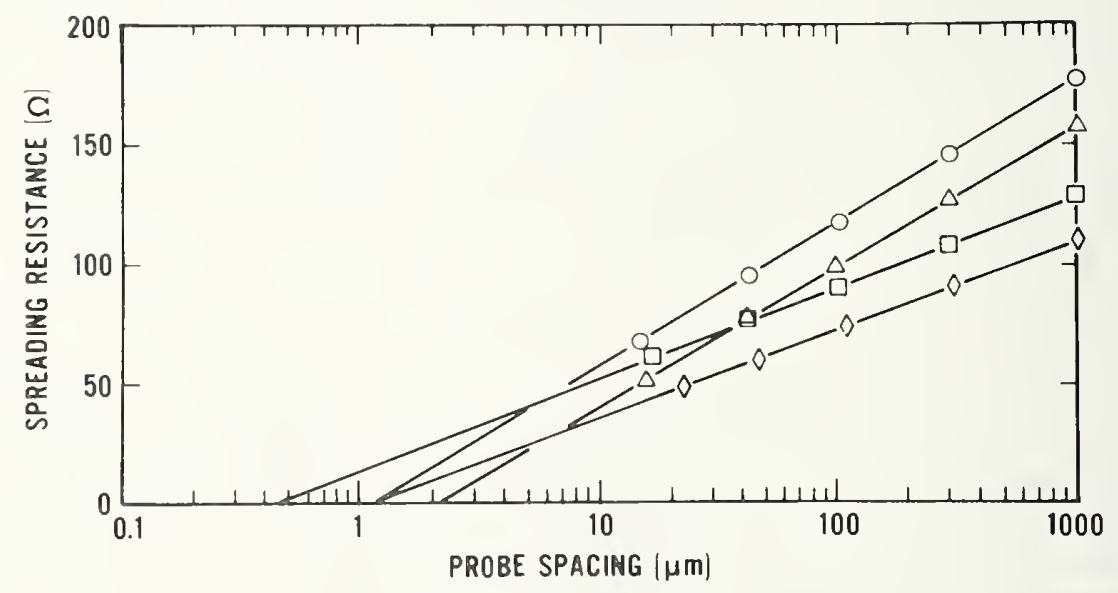

(b)

Figure 6. Spreading resistance vs. probe spacing for junction-isolated layers. Layer-type and four-probe sheet resistance values are: (a) O, p-type, $284 \Omega / 0 ; 0$; p-type, $126 \Omega / \square ; \Delta, p$-type, $56.4 \Omega / \square ;$ (b) 0 and $\Delta$, p-type, $76.4 \Omega / \square ; 0$ and $\diamond, n$-type, $52.0 \Omega / 0$. All data for figure $6 a$ and for triangle and diamond in figure $6 \mathrm{~b}$ taken at $20 \mathrm{-g}$ probe load; data for circle and square in figure $6 \mathrm{~b}$ taken at $10 \mathrm{-g}$ load. 
Table 1. Comparison of Diffused Layer Sheet Resistance Values for Three Boron Diffusions.

Specimen

Sheet Resistance, $\Omega / \square$

Four Probe

Two Probe

In (s) Dependencea

\begin{tabular}{lrr}
\hline Boron diffusion, $x_{j}=0.7 \mu \mathrm{m}^{\mathrm{b}}$ & 284 & 250 \\
Boron diffusion, $\mathrm{x}_{\mathrm{j}}=1.6 \mu \mathrm{m} c$ & 126 & 126 \\
Boron diffusion, $\mathrm{x}_{\mathrm{j}}=4.3 \mu \mathrm{m}$ & 54.6 & 52
\end{tabular}

a Two-probe data are shown in figure $6 \mathrm{a}$.

b Profile data for this diffusion are shown in figure 7 .

c Profile data for this diffusion are shown in figure 8.

d Profile data for this diffusion are shown in figure 9.

Table 2. Comparison of Diffused Layer Sheet Resistance Values for One $n$-Type Diffusion and One p-Type Diffusion.

\begin{tabular}{lcc}
\hline Specimen & Four Probe & $\begin{array}{c}\text { Sheet Resistance, } \Omega / \square \\
\text { (average of } 10 \mathrm{~g} \text { and 20 g data) }\end{array}$ \\
\hline Boron diffusion ${ }^{\text {Two }}$ & 76.4 & 81.9 \\
Phosphorus diffusion $\mathrm{c}$ & 52.0 & 53.3 \\
\hline
\end{tabular}

a Two-probe $\ln (\mathrm{s})$ dependence is shown in figure $6 \mathrm{~b}$.

b

Profile data for this diffusion are shown in figure 10.

C Profile data for this diffusion are shown in figure 14. 
found to be closely related to the sheet resistance of the layer. The result is a verification of eq (8), and by implication, the difference equations which lead to eq (16).

It should be noted that the consistency of the data down to the smallest spacing shown corresponding to $\mathrm{s} / \mathrm{a}$ ratios as small as 7 or 8 , implies that the basic model for isolated layers is valid down to s/a ratios of this order. Hence, any volume correction factors derived from the model should be applicable to data taken with such s/a values. However, preliminary data, not shown, indicate a breakdown of the linear dependence of $\mathrm{R}$ on $\ln (\mathrm{s})$ when using probe spacing on the order of 6 to $8 \mu \mathrm{m}$, corresponding to $\mathrm{s} / \mathrm{a}$ values as small as 4 or 5 . Use of volume correction factors as derived here are questionable under such s/a measurement conditions.

\subsection{Diffusion Profiles}

The sampling volume correction schemes described above were tested in a series of experiments. Test specimens containing relatively shallow diffusions were prepared, and spreading resistance profiles of the diffused regions were generated. Two sets of specimens were used in these experiments, each prepared to test a particular volume correction equation. The conductivity profiles corrected as discussed below were then compared with profiles generated using the incremental sheet resistance method, ISR, on adjacent sections of the same specimens. The results generally substantiate the validity of the correction schemes, but a number of experimental effects (primarily angle effects, see sec. 2.3) not accounted for in the theory here are evident in the comparisons.

The first set was a series of boron diffusions having a wide range of junction depths $(0.7,1.6$, and $4.3 \mu \mathrm{m}$, as measured by angle lap and stain) and nominally constant surface concentration (about $1 \times 10^{19}$ atoms $\mathrm{cm}^{-3}$ ), the substrate being $10 \Omega \cdot \mathrm{cm} n$-type silicon. This set was intended to provide a range of conductivity gradients and layer thicknesses to test the range of validity of the concept of parallel superposition.

The conductivity profiles obtained on this set of specimens are shown in figures 7 to 9. The points shown were derived from spreading resistance data using the parallel superposition formula, eq (16). The procedure is to first estimate the slope at the deepest point still above the junction, calculate a correction factor using only the first term in eq (16), adjust the measured resistance at that point by the factor F, and look up the conductivity corresponding to the adjusted resistance on the calibration curve generated from a sequence of bulk silicon specimens with known resistivity (conductivity), having the proper crystallographic orientation. The conductivity value thus obtained is plotted on the final graph for the datum in question and is also used in the second term of eq (16) when the correction factor is calculated for each succeeding shallower point. For each succeeding point (working toward the surface), an additional term appears in the summation term of eq (16), so when the point at the surface is considered, the summation is 


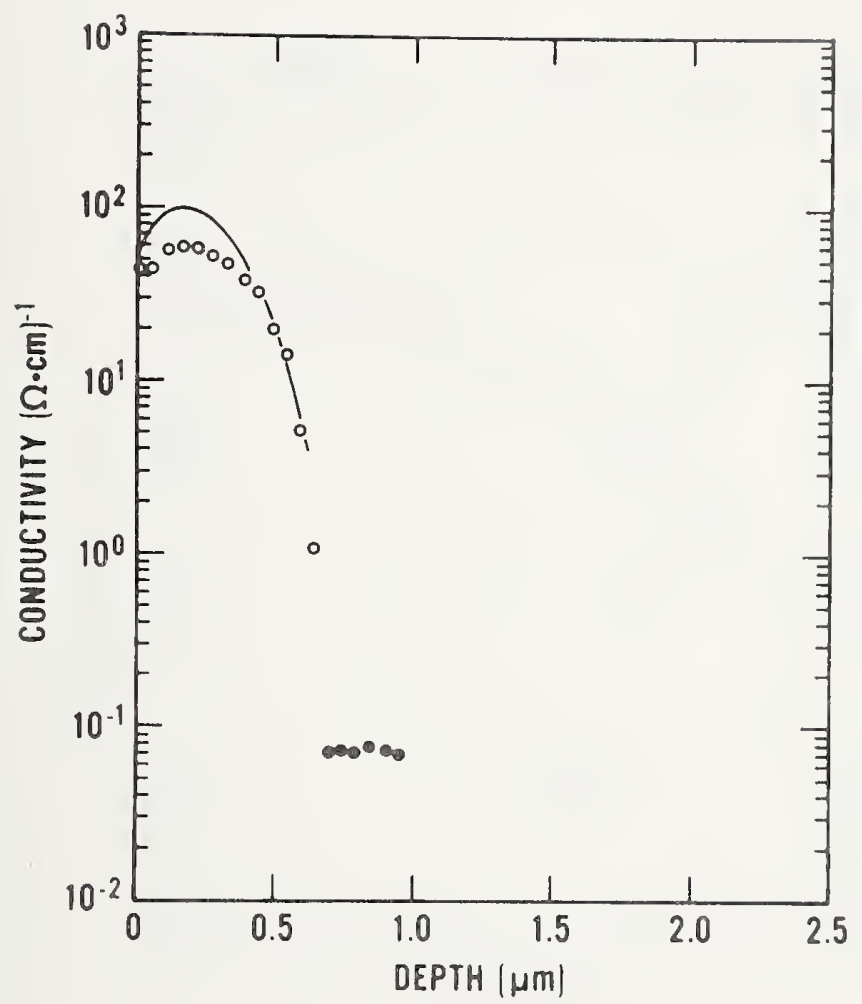

Figure 7. Conductivity profile for boron diffusion into nominal $10-\Omega \cdot \mathrm{cm} n$-type substrate. Nominal surface density of boron, $10^{19} \mathrm{~cm}^{-3}$; junction depth by lap and stain, 0.7 $\mu \mathrm{m}$. Open circles, spreading resistance data corrected using parellel superposition, eq (16b); solid circles, uncorrected spreading resistance data; solid line, smoothed incremental sheet resistance data. 


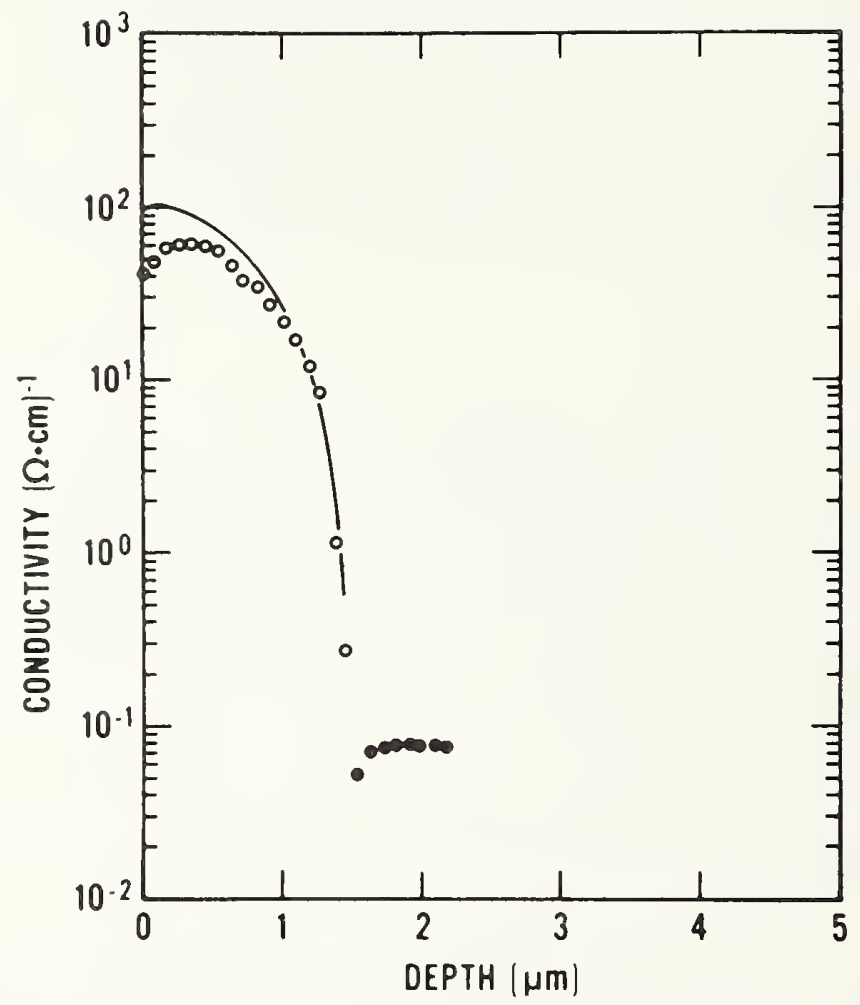

Figure 8. Conductivity profile for boron diffusion into nominal $10-\Omega \cdot \mathrm{cm} n$-type substrate. Nominal surface density of boron, $10^{19} \mathrm{~cm}^{-3}$; junction depth by lap and stain, 1.6 $\mu \mathrm{m}$. Open circles, spreading resistance data corrected using parallel superposition, eq (16b); solid circles, uncorrected spreading resistance data; solid line, smoothed incremental sheet resistance data. 


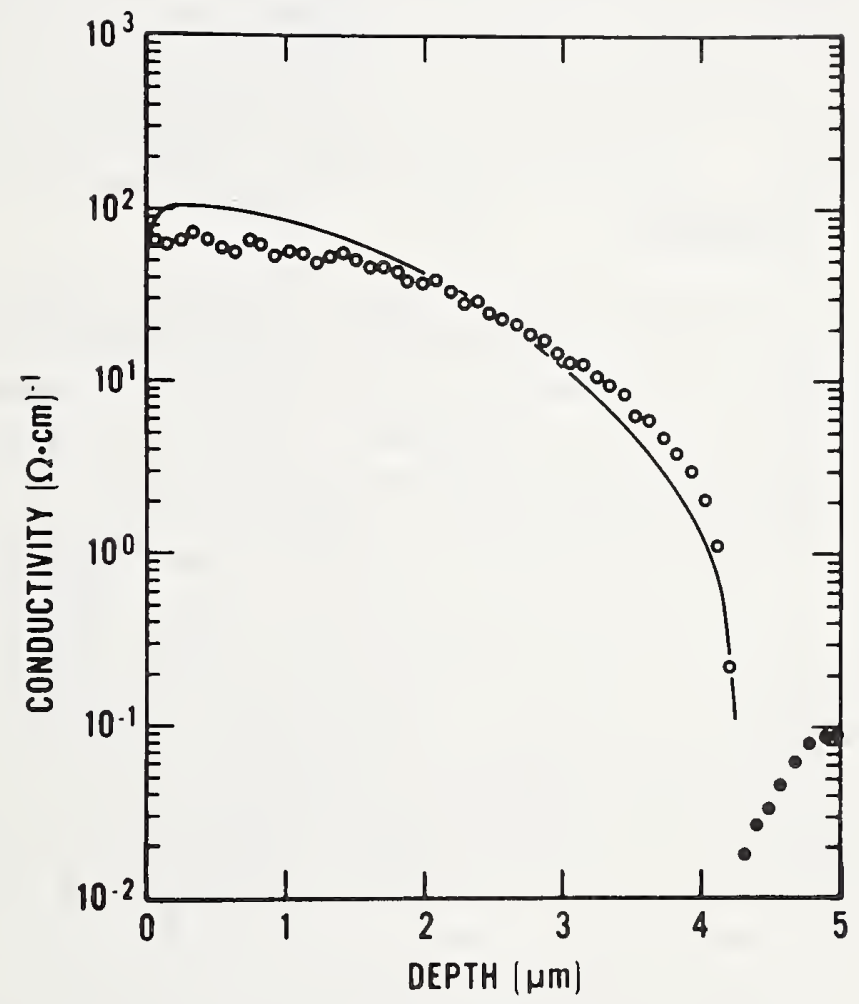

Figure 9. Conductivity profile for boron diffusion into nominal $10-\Omega \cdot \mathrm{cm} n$-type substrate. Nominal surface density of boron, $10^{19} \mathrm{~cm}^{-3}$; junction depth by lap and stain, 4.3 $\mu \mathrm{m}$. Open circles, spreading resistance data corrected using parallel superposition, eq (16b); solid circles, uncorrected spreading resistance data; solid line, smoothed incremental sheet resistance data. 
over all points between the junction and the surface. Appendix B contains further details of the calculations. Simple analytic expressions are used as approximations for $\mathrm{H}$ and its second derivative and are described near the end of Appendix A.

In each case for these first three specimens, the spreading resistance profile lies below the incremental sheet resistance (ISR) profile near the surface but coincides closely at deeper points. The discrepancy is thought to be related to bevel-angle effects [5]. Such effects, which are absent in the ISR experiments, are not accounted for in the data reduction scheme used here. The general agreement between the two methods is taken as proof of the viability of the superposition scheme for specimens with an isolating junction.

The second set of diffusions was prepared on substrates of widely different resistivity but with nominally constant profile shape and depth. This set was used to determine what effects the presence of a low resistivity substrate below the junction would have on the corrected profiles. Profiles for this set of specimens are shown in figures 10 to 17 and a summary of profile parameters is given in table 3 . This set of specimens consisted of four boron-diffused wafers and four phosphorusdiffused wafers. The boron diffusions were made under the same diffusion conditions into three $n$-type substrates of nominal $0.03,1.0$, and $150 \Omega \cdot \mathrm{cm}$ resistivity and into one p-type substrate of nominal $100 \Omega \cdot \mathrm{cm}$ resistivity. The phosphorus diffusions were made under the same diffusion conditions into p-type substrates of nominal $0.03,1.0$, and $100 \Omega \cdot \mathrm{cm}$ resistivity and into one $n$-type substrate of nominal $150 \Omega \cdot \mathrm{cm}$ resistivity. Each of the spreading resistance-based profiles was generated. using the local slope formula, eq (22). This formula yields results almost indistinguishable from those obtained from parallel superposition, not shown, particularly for points less than one or two contact radii from a junction. The agreement with the ISR profiles was generally the same as was found with the first set of specimens: the spreading resistance profiles tend to be low near the surface, especially on p-type layers. The depth scales do not coincide exactly in all cases, owing to experimental errox in both measurements and to the fact that slight variations in junction depth occurred in the different regions of the specimen analyzed.

Comparing figures 10 and 13 for boron diffusions, and figures 14 and 17 for phosphorus diffusions, a very good agreement is seen in each pair for profile shape and absolute value from the surface to within a few points of the junction. This is taken as an indication that the local slope method works quite well even when the substrate does not approach one of the limiting cases in the starting models.

The computer program used for reduction of the spreading resistance data is described and listed in Appendix B. It incorporates both eqs (16) and (22) as separate routines and includes data logging and calibration curve look-up routines. The program does not include raw data smoothing so the results may suffer from random variations in the data. Execution times for the local slope routine are on the order of three 


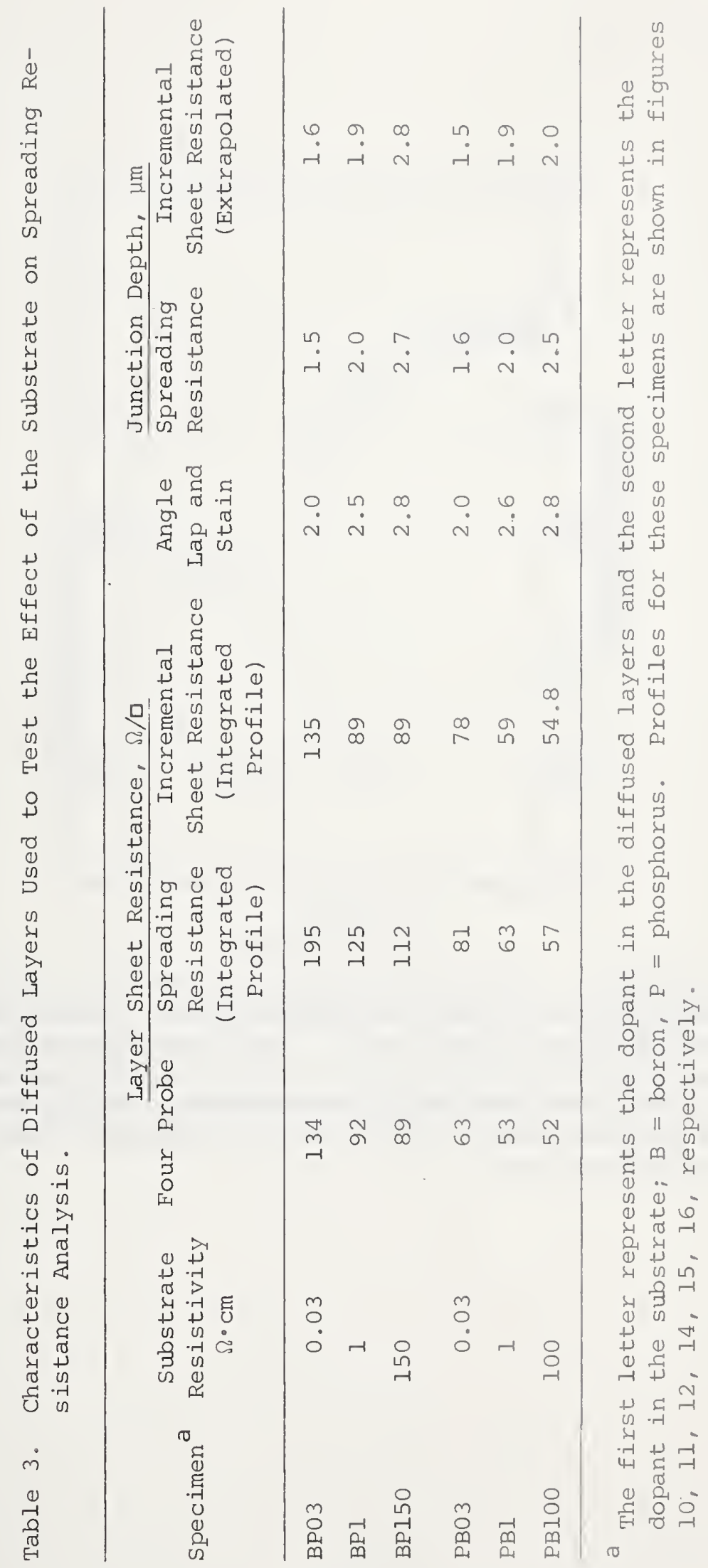




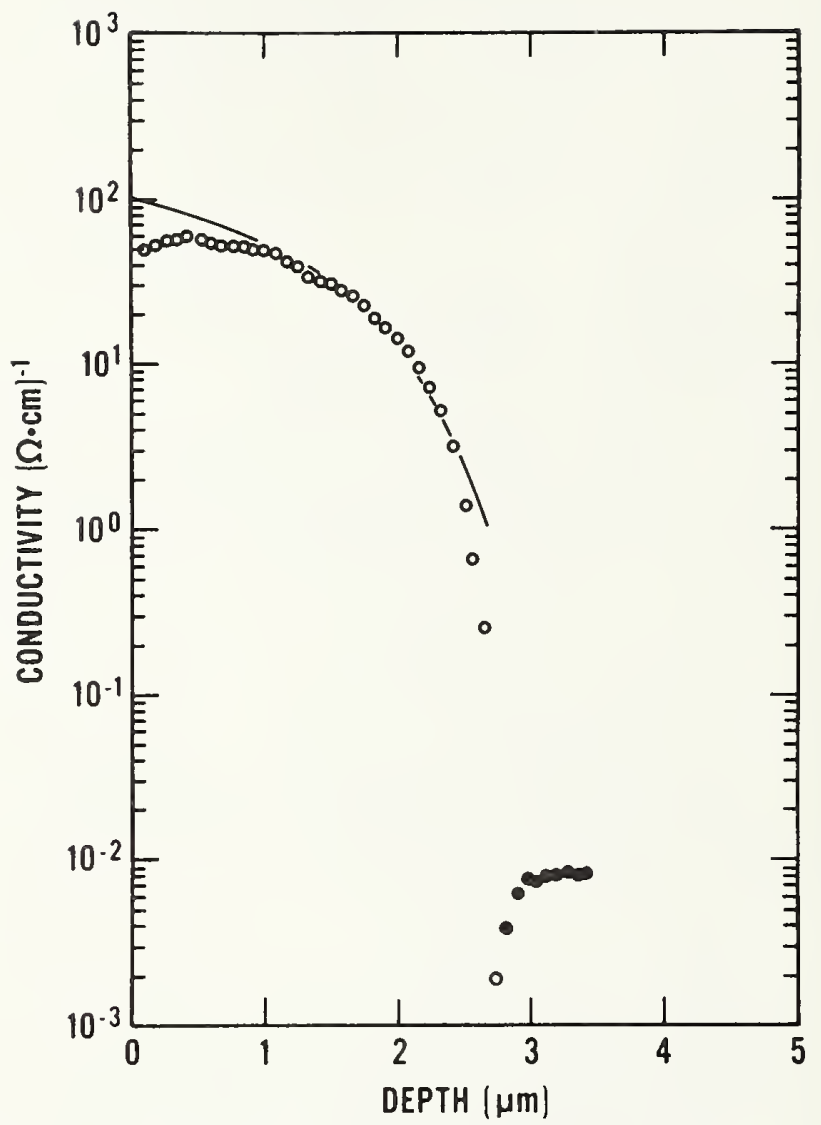

Figure 10. Conductivity profile for boron diffusion into nominal $150-\Omega \cdot \mathrm{cm} n$-type substrate. Nominal boron surface density, $10^{19} \mathrm{~cm}^{-3}$. Open circles, spreading resistance data corrected by the local slope method, eq $(16 \mathrm{~b})$; solid circles, uncorrected spreading resistance data; solid line, smoothed incremental sheet resistance data. 


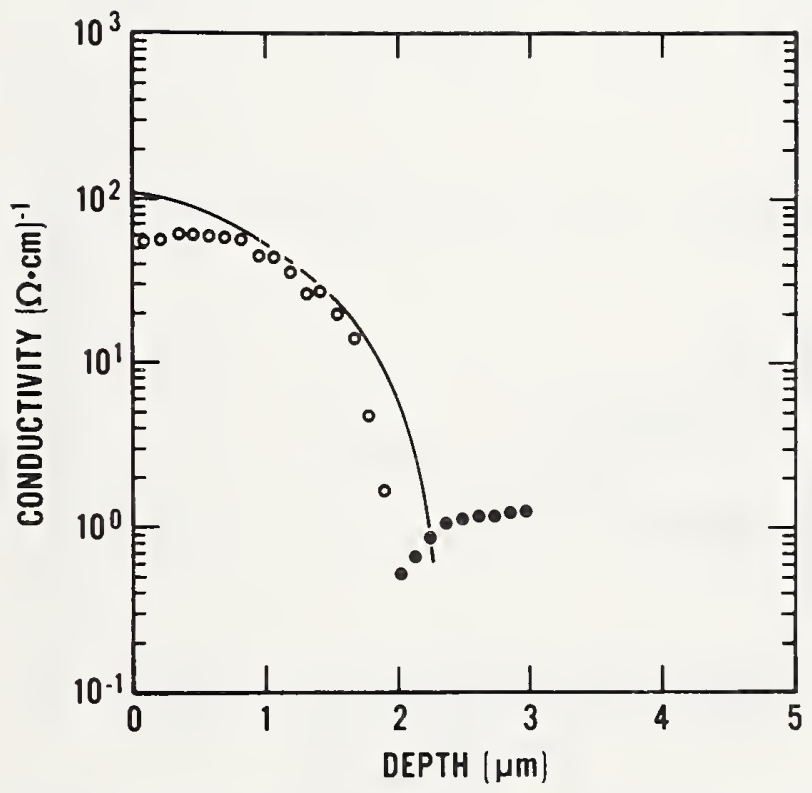

Figure 11. Conductivity profile for boron diffusion into nominal $1.0-\Omega \cdot \mathrm{cm} n$-type substrate. Nominal boron surface density, $10^{1 y} \mathrm{~cm}^{-3}$. Open circles, spreading resistance data corrected by the local slope method; solid circles, uncorrected spreading resistance data; solid line, smoothed incremental sheet resistance data. 


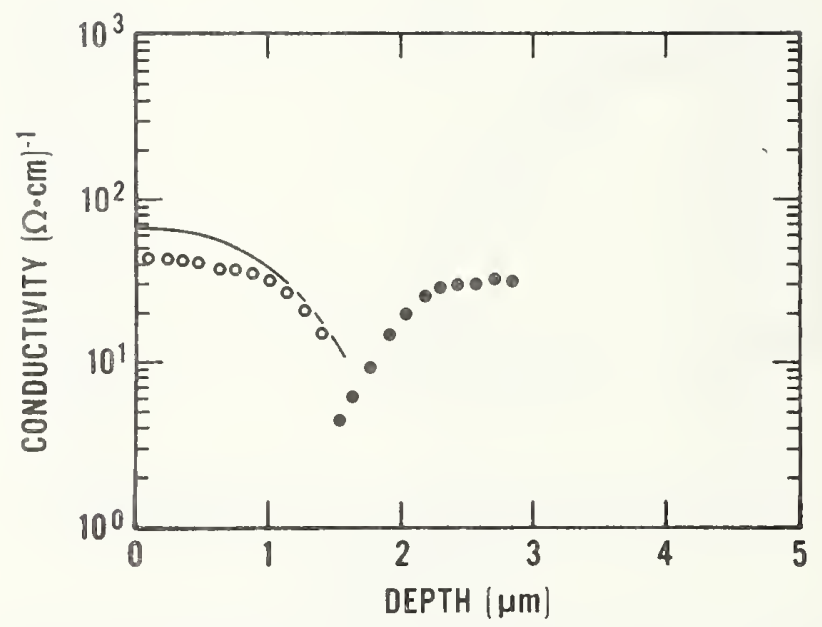

Figure 12. Conductivity profile for boron diffusion into nominal $0.03-\Omega \cdot \mathrm{cm} n$-type substrate. Nominal boron surface density, $10^{19} \mathrm{~cm}^{-3}$. Open circles, spreading resistance data corrected by the local slope method; solid circles, uncorrected spreading resistance data; solid line, smoothed incremental sheet resistance data. 


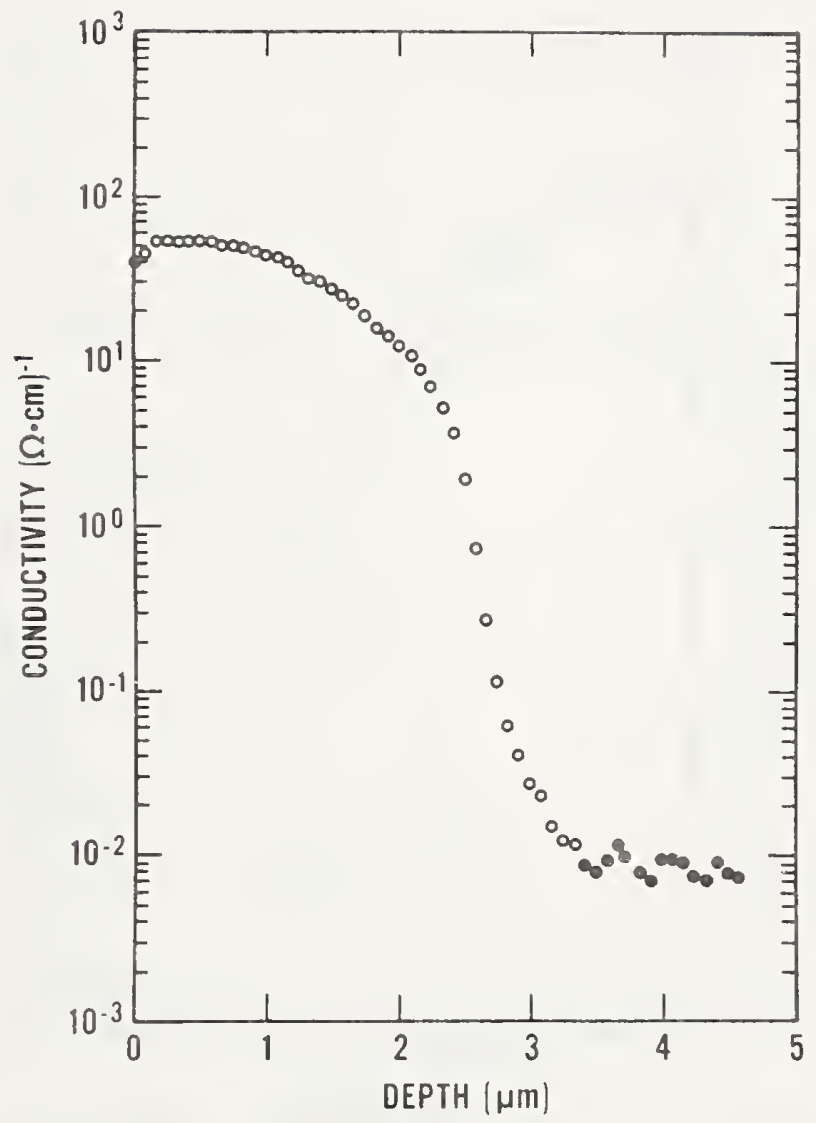

Figure 13. Conductivity profile for boron diffusion into nominal $100-\Omega \cdot \mathrm{cm}$ p-type substrate. Nominal boron surface density, $10^{19} \mathrm{~cm}^{-3}$. Open circles, spreading resistance data corrected by the local slope method; solid circles, uncorrected spreading resistance data. Compare with figure 10 for effect of noninsulating boundary on profile interpretation. 


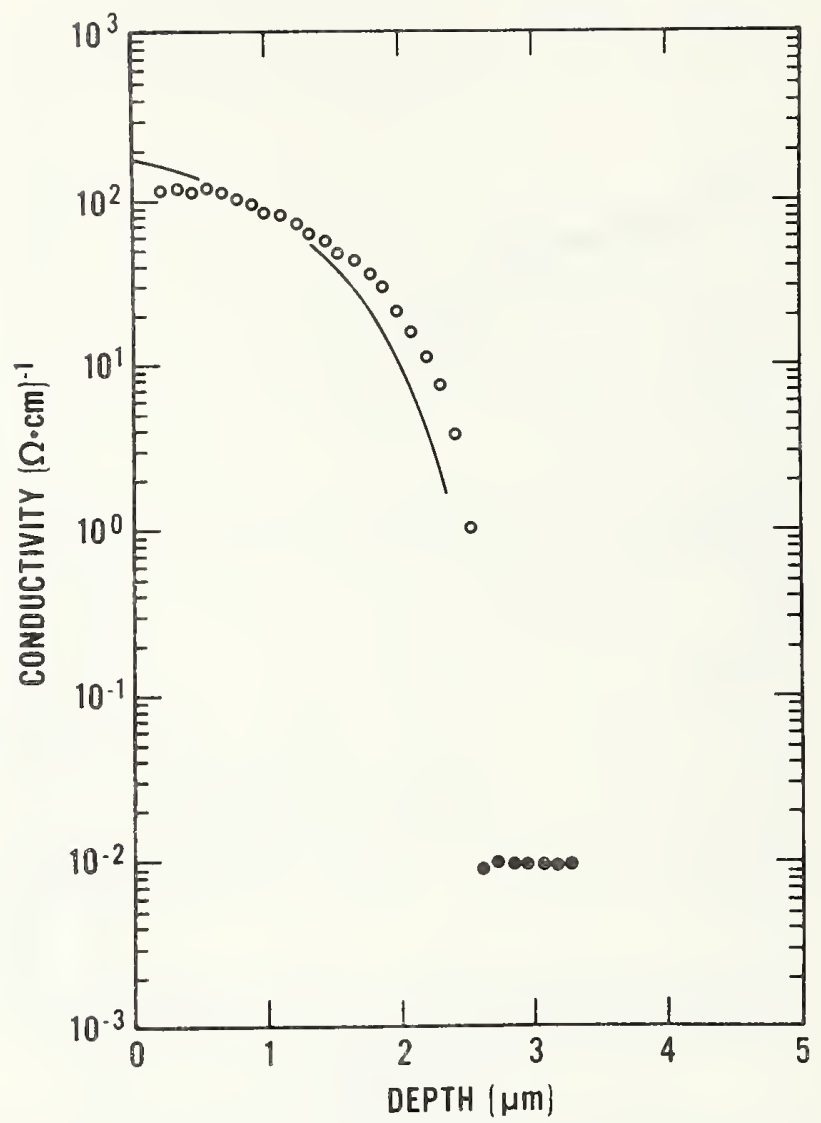

Figure 14. Conductivity profile for phosphorus diffusion into nominal $100-\Omega \cdot \mathrm{cm}$ p-type substrate. Nominal phosphorus surface density, $10^{19} \mathrm{~cm}^{-3}$. Open circles, spreading resistance data corrected by the local slope method; solid circles, uncorrected spreading resistance; solid line, smoothed incremental sheet resistance data. 


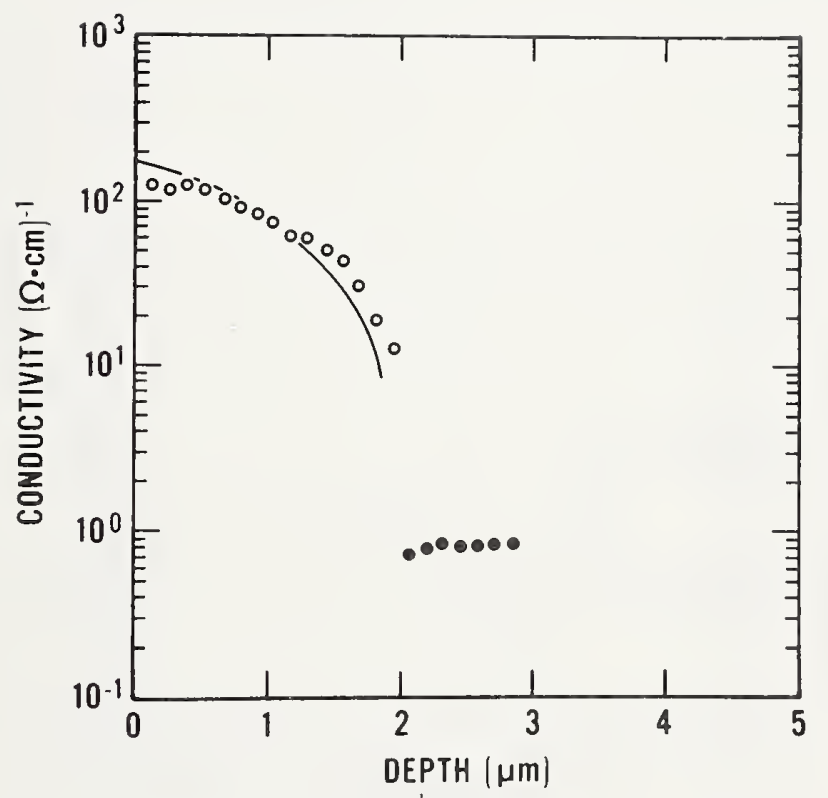

Figure 15. Conductivity profile for phosphorus diffusion into nominal $1.0-\Omega \cdot \mathrm{cm}$-type substrate. Nominal phosphorus surface density, $10^{19} \mathrm{~cm}^{-3}$. Open circles, spreading resistance data corrected by the local slope method; solid circles, uncorrected spreading resistance; solid line, smoothed incremental sheet resistance data. 


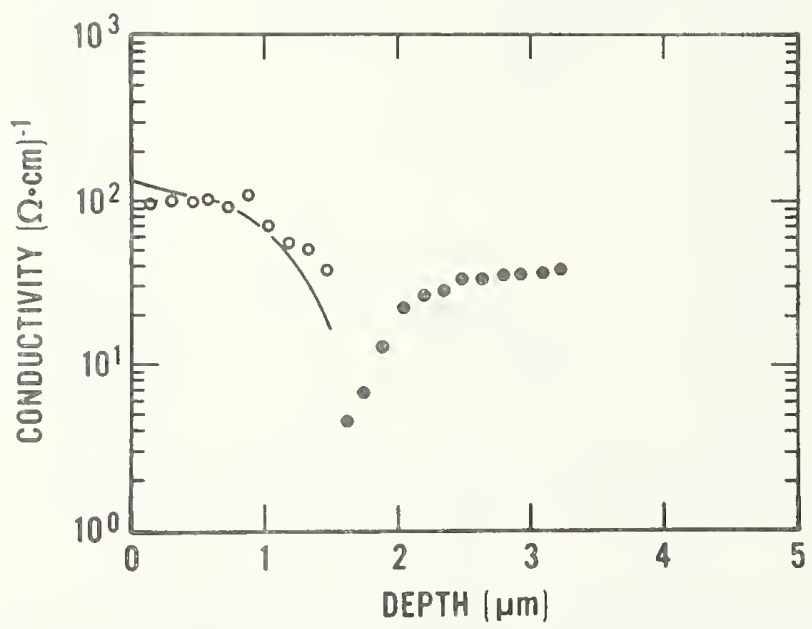

Figure 16. Conductivity profile for phosphorus diffusion into nominal $0.03-\Omega \cdot \mathrm{cm} p$-type substrate. Nominal phosphorus surface density, $10^{19} \mathrm{~cm}^{-3}$. Open circles, spreading resistance data corrected by the local slope method; solid circles, uncorrected spreading resistance; solid line, smoothed incremental sheet resistance data. 


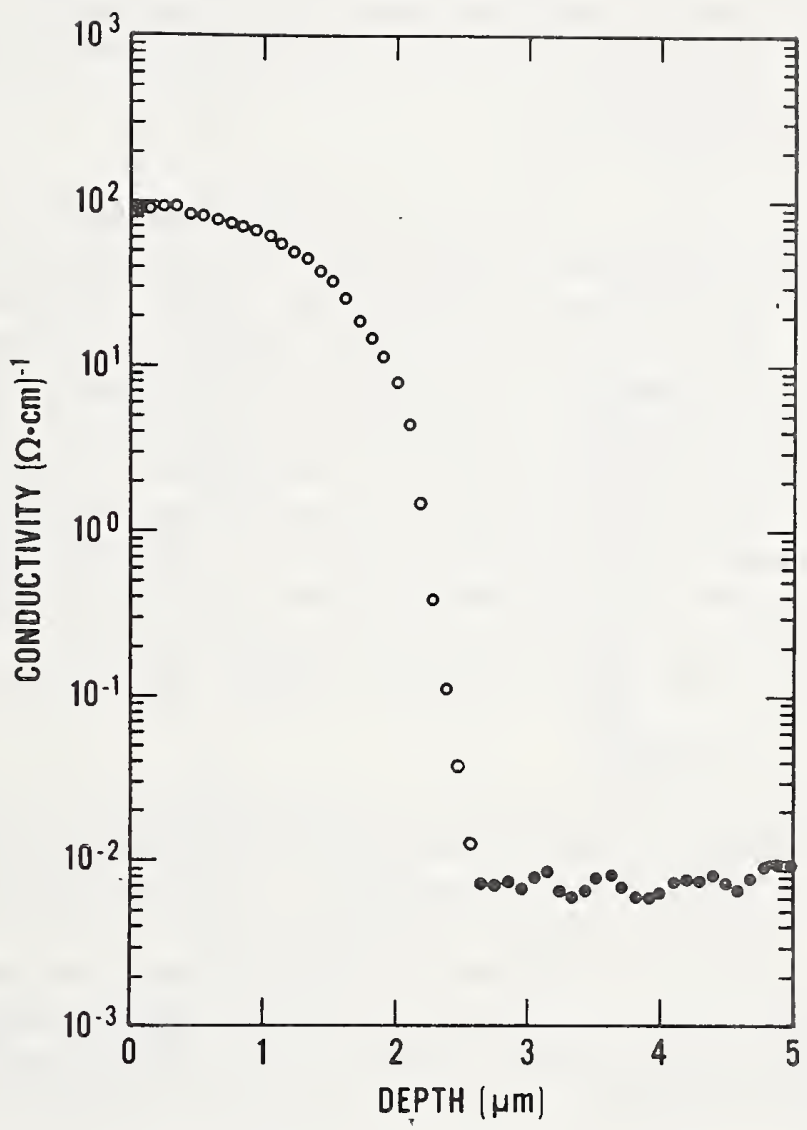

Figure 17. Conductivity profile for phosphorus diffusion into nominal $150-\Omega \cdot \mathrm{cm} n$-type substrate. Nominal phosphorus surface density, $10^{19} \mathrm{~cm}^{-3}$. Open circles, spreading resistance data corrected by the local slope method; solid circles, uncorrected spreading resistance. Compare with figure 14 for effect of noninsulating boundary on profile interpretation. 
seconds per point for the Hewlett-Packard Model $9830 \mathrm{~A}$ programmable calculator. For the parallel superposition routine, times run as high as $10 \mathrm{~s}$ for the last few points on a 40-point profile. As can be judged from the simplicity of the program listed in Appendix B, a relatively inexpensive machine can be used for data reduction and so can be considered as a dedicated accessory to the spreading resistance probe.

\subsection{Bevel-Edge Proximity Effect}

When the original top surface of a beveled specimen is probed, an increase in measured spreading resistance is often noted as the bevel edge is approached. The effect is most noticeable on shallow diffusions and depends on the spacing between the two probes. The effect is an obvious illustration of asymmetry introduced by beveling away one side of the specimen. A theoretical treatment of the effect is an extremely complicated task involving conductivity gradients below the surface, bevel angle and location, and probe spacing. The interrelation of angle and spacing was investigated briefly on one of the specimens used for the profiling experiments. The results are shown in figure 18 where spreading resistance on the original surface is plotted versus distance from the bevel edge for two bevel angles and two values of probe spacing.

A partial understanding of the bevel-edge proximity effect can be gained by considering the simple case of a uniform-resistivity wedgeshaped specimen. A theoretical treatment of this problem is given in Appendix C. The results of this investigation show how the uniformlayer correction factor is affected by nonparallel top and bottom surfaces of the classic infinite slab. As in the experimental investigation above, the effect is minimized with small probe spacing and, of course, vanishes as the bevel angle approaches zero.

\section{CONCLUSIONS}

The exact solution of the boundary value problem associated with a spreading resistance experiment is an exceedingly difficult task. Experimental problems, such as barrier resistance effects, probe calibration, and probe stability, introduce errors which make the accuracy of computations difficult to verify. This work has shown that simplifying the theoretical model can accrue computational savings which far outweigh the loss of accuracy involved. The mathematical treatments of limiting-case parallel and series models have served to provide proportionality constants which tie the empirical local slope scheme to a physical model.

The probe spacing experiment has two main values: it demonstrates that spreading resistance on a thin, insulated structure is closely related to sheet resistance, and it provides an experimental value for contact radius which is a necessary parameter in the calculations.

The diffusion profile experiments, while demonstrating the viability of the correction schemes, serve better to illustrate the need for 


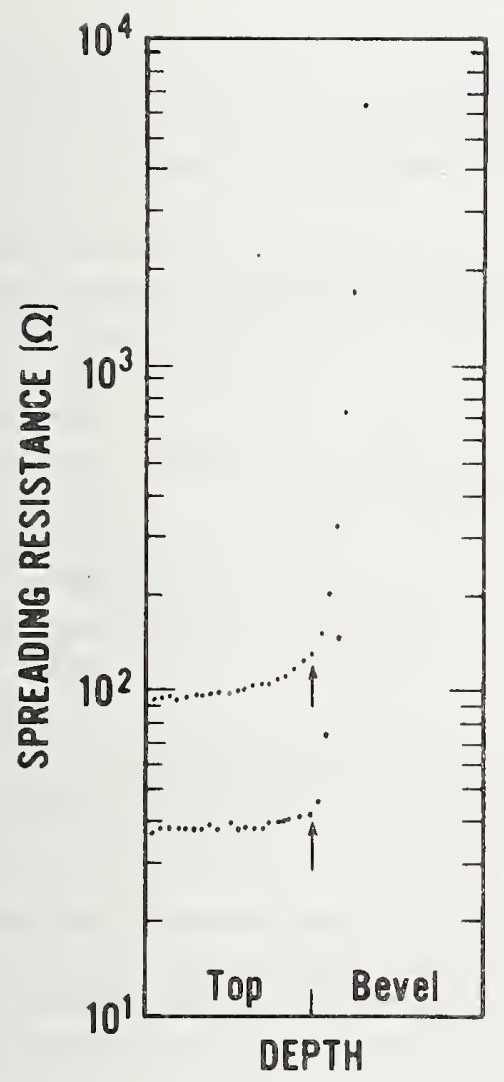

a. Bevel angle, 0.05 rad; upper trace probe spacing, 200 Hm; lower trace probe spacing, $12 \mu \mathrm{m}$.

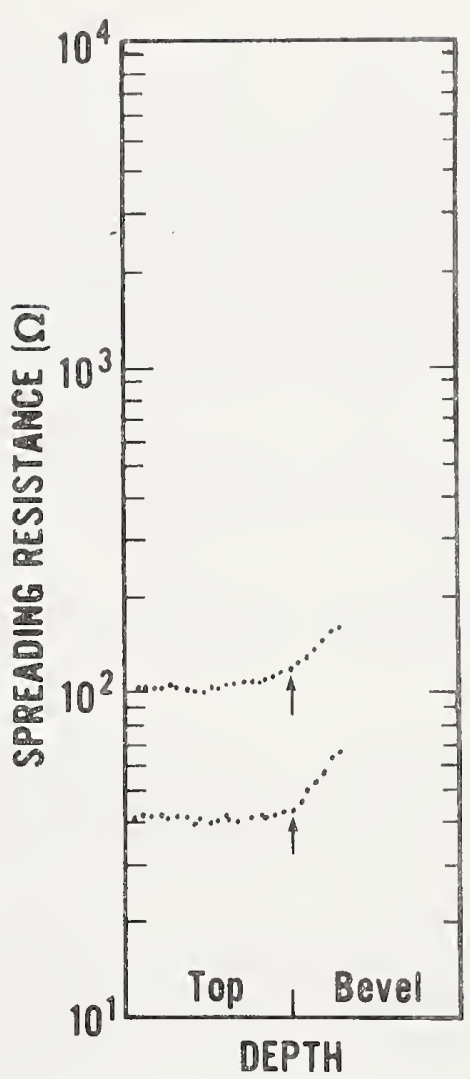

b. Bevel angle, 0.01 rad; upper trace probe spacing, $200 \mu \mathrm{m}$; lower trace probe spacing, $12 \mu \mathrm{m}$.

Figure 18. Spreading resistance in the vicinity of the bevel edge. Specimen same as shown in figure 10. Original top surface extends to left of demarcator. Step size is $10 \mu \mathrm{m}$. 
further understanding of bevel-angle effects. A recent investigation [5] has shown that if spreading resistance data are taken on an anodically sectioned (rather than beveled) specimen, much of the discrepancy of the type observed near the top of the layer will disappear.

\section{REFERENCES}

1. Schumann, P. A., and Gardner, E. E., Application of Multilayer Potential Distribution to Spreading Resistance Correction Factors, J. Electrochem. Soc. 116, 87-91 (1969).

2. Yeh, T. H., and Khokhani, K. H., Multilayer Theory of Correction Factors for Spreading Resistance Measurements, J. Electrochem. Soc. 116, 1461-1464 (1969).

3. Hu, S. M., Calculation of Spreading Resistance Correction Factors, Solid-State Electronics 15, 809-817 (1972).

4. Choo, S. C., Leong, M. S., and Kuan, K. L., On the Calculation of Spreading Resistance Correction Factors, Solid-State Electronics 19, 561-565 (1976).

5. D'Avonzo, D. C., and Dutton, R. W., Spreading Resistance for Impurity Profiles, Technical Report No. 5013-2, Stanford Electronics Laboratories, Stanford University (February 1977).

6. Pinchon, P. M., On the Validity of Correction Factors Applied to Spreading Resistance Measurements on Beveled Structures, Semiconductor Measurement Technology: Spreading Resistance Symposium, J. R. Ehrstein, Ed, NBS Special Publication 400-10, pp. 51-61 (December 1974).

7. Holm, R., Electric Contacts, 4th ed., p. 16 (Springer-Verlag, New York, 1967).

8. Dickey, D. H., Two-Point Probe Correction Factors, Semiconductor Measurement Technology: Spreading Resistance Symposium, J.R. Ehrstein, Ed, NBS Special Publication 400-10, pp. 45-50 (December 1974).

9. Tannenbaum, E., Detailed Analysis of Thin Phosphorus Diffusions in p-type Silicon, Solid-State Electronics 2, 123-132 (1961).

10. Spiegel, M. R., Calculus of Finite Differences and Difference Equations, p. 3, Schaum's Outline Series (McGraw-Hill, New York, 1971).

11. Jackson, J. D., Classical Electrodynamics, p. 64 (Wiley, New York, 1962).

12. Ibid., p. 92 . 
APPENDIX A

COMPUTER EXERCISES TO COMPARE CORRECTION FACTOR METHODOLOGIES

\section{A.1 Introduction}

The exercises described here were designed to test the two schemes developed: parallel superposition for isolated layers only and local slope for general layer configurations.

In Exercise I, section A.2, the correction factor from parallel superposition is compared with factors calculated using the method of images. Here a single thin layer of uniform conductivity on an infinitely thick substrate of lower conductivity is considered. In Exercises II and III, section A.3, both parallel superposition and local slope are compared with the results from the Schumann-Gardner (SG) approach. In Exercise II, they are compared for two uniform layers on an infinite substrate and, in Exercise III, for three layers on an insulating substrate. (The second exercise, wherein the bottom-layer thickness is taken as very large, is a special case of the third.)

A.2 Exercise I - Parallel Superposition versus Image Solution - Based on Current Admixture

For this exercise, the correction factor evaluated using the parallel superposition formalism is compared with a correction factor derived directly from the method of images. This latter correction incorporates finite size contacts and a more general current distribution than used by SG. This more general current distribution allows calculations that reasonably guarantee, not merely assume, constant potential value under the contacts. In this way, parallel superposition is tested with the most generalized physical parameters yet developed which meet the basic boundary conditions. In order to keep the image solution tractable, however, the model is limited to two layers.

The image solution using current admixture is constructed as follows: the radial potential distribution for one of the contact disks is derived for two types of current distribution over the disk, one being a ringshaped distribution in which the current is a delta function at the periphery of the disk (correct for a very thin, isolated single layer), and the other being the classical distribution appropriate for a semi-infinite uniform solid. The radial potential for unit current of each distribution is calculated and admixed to achieve an approximately constant radial potential. The model used for this first exercise is shown in figure Al. By taking the substrate conductivity as zero in this model, the result is the correction factor for an isolated slab of arbitrary thickness, and subsequent results (for various thicknesses) are then used to calculate factors for arbitrary substrate conductivity from parallel superposition.

Given a ring-shaped current of radius, a, imbedded in a medium with uniform resistivity and located a distance, $z$, above the origin, the po- 


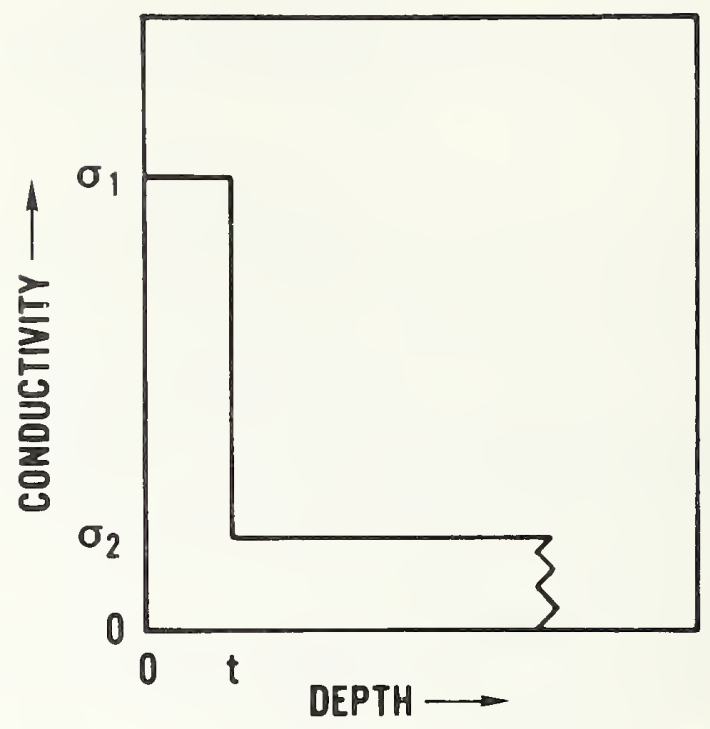

Figure Al. Schematic conductivity profile for Computer Exercise I.

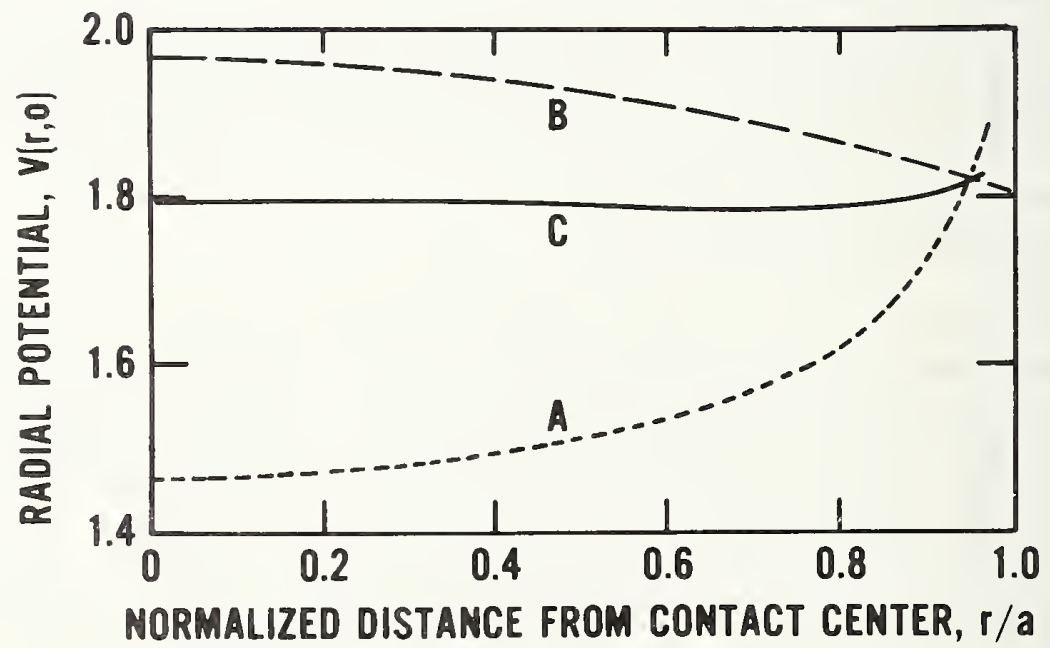

Figure A2. Radial potential distribution on a spreading resistance contact. Structure of figure $A l$ with $t=0.3 \mathrm{a}$ and $\beta=0.5\left(\sigma_{1}=3 \sigma_{2}\right)$. Curve $A$ : from the ring-shaped delta function current distribution, eq (A3); curve B: from current distribution appropriate to semi-infinite substrate, eq (A4) (this is the distribution used by $S G$ ); curve C: from admixture of current distributions above. The admixture coefficient is chosen to force $V_{C}(0)$ to equal $V_{C}(r / a=0.9)$ with the result for this structure $\mathrm{V}_{C}=(1-0.659) \mathrm{V}_{\mathrm{A}}+0.659 \mathrm{v}_{\mathrm{B}}$. 
tential $V(r, z)$ on the $z=0$ plane may be written in terms of Legendre polynomials [11]:

$$
V(r, 0)=\frac{I \rho}{\pi \sqrt{a^{2}+z^{2}}} \sum_{l=0}^{\infty}\left(\frac{4}{\sqrt{a^{2}+z^{2}}}\right)^{2 l} P_{2 l}\left(\frac{z}{\sqrt{a^{2}+z^{2}}}\right) P_{2 l}(0)
$$

For a ring-shaped current on the surface of the model of figure $A 1$, the potential inside the ring can be written as a sum of terms, each of the same form as eq (Al), where each term of the sum corresponds to an image source. If the real source has strength $I$, the first image pair (at a distance $\mathrm{z}= \pm 2 \mathrm{Nt}$ ) will have strength $\beta I$, where

$$
\beta=\frac{\sigma_{1}-\sigma_{2}}{\sigma_{1}+\sigma_{2}} \text {. }
$$

Each succeeding image pair will be weaker by the factor $\beta$. If the substrate has zero conductivity, $\beta$ is unity and each image has the same strength as the source. The second contact, displaced a distance s, also has a series of images of similar strength associated with it. For simplicity, using $s=40 \mathrm{a}$, the second contact and its images can be assumed to be point-like and a constant potential is subtracted to account for them. The radial potential then, inside the first current ring, is given by

$V(r, 0)=\frac{I \rho}{2 \pi a}\left\{\sum_{\ell=0}^{\infty}\left(\frac{r}{a}\right)^{2} l\left[P_{l}(0)\right]^{2}-\frac{a}{s}\right\}$

$+\frac{I \rho}{\pi} \sum_{N=1}^{\infty}\left(\frac{\beta^{N}}{\sqrt{a^{2}+(2 N t)^{2}}} \sum_{l=0}^{\infty}\left(\frac{r}{\sqrt{a^{2}+(2 N t)^{2}}}\right)^{2 l} P_{2 l}\left(\frac{2 N t}{\sqrt{a^{2}+(2 N t)^{2}}}\right) P_{2 l}\right.$

$\left.-\frac{\beta^{N}}{\sqrt{s^{2}+(2 N t)^{2}}}\right\}$

This expression has been summed numerically for various values of $r, t$, and $\beta$. The results for $t=0.3 a$ and $\beta=0.5$ are shown as curve $A$ in figure A2.

The second current distribution to be considered is that appropriate to an equipotential disk contact on a semi-infinite uniform solid, namely:

$$
J(r)=\frac{I}{2 \pi a^{2}}\left[1-(r / a)^{2}\right]^{-1 / 2} .
$$


Given this distribution, the potential at any point in the solid is [1I]

$$
V(r, z)=\frac{I}{2 \pi a} \sin ^{-1}\left[\frac{2 a}{\sqrt{(r-a)^{2}+z^{2}}+\sqrt{(r+a)^{2}+z^{2}}}\right]
$$

This current distribution is applied to the two-layer model structure, using the same image strengths as before; the radial potential on the first contact, $z=0$, is written as

$$
\begin{aligned}
V(r, 0) & =\frac{I \rho}{4 a}\left\{I+\frac{4}{\pi} \sum_{N=1}^{\infty} \beta^{N} \sin ^{-1}\left[\frac{2 a}{\sqrt{(r-a)^{2}+(2 N t)^{2}}+\sqrt{(r+s)^{2}+(2 N t)^{2}}}\right]\right. \\
& \left.-\frac{2 a}{\pi s}-\frac{2}{\pi} \sum_{N=1}^{\infty} \beta^{N} \frac{a}{\sqrt{s^{2}+(2 N t)^{2}}}\right\}
\end{aligned}
$$

where the second contact and its images are again assumed to be point sources. This expression has also been summed numerically; the results for $t=0.3 \mathrm{a}$ and $\beta=0.5$ are shown as curve $B$ in figure A2. (It is this radial potential which is averaged over the surface of the disk in the SG approach.)

There is not a linear current admixture, using the two types of current distribution considered above, which will result in a constant radial potential under the contact for any particular values of $t$ and $\beta$. Nevertheless, the requirement of constant potential can be approximated: mixtures of the two (keeping unit total current) have been taken which force the potential at $r=0.9 \mathrm{a}$ to equal the potential at $r=0$. The result of such admixture for $t=0.32$ and $\beta=0.5$ is shown as curve $C$ in figure A2. The difference between curve B, the SG distribution, and curve $C$ suggests that noticeable errors may occur in the SG procedure as a result of considering only the current distribution appropriate to a semi-infinite solid, when evaluating more general structures. As will be shown in section A.4 of this appendix, this error is small, at least for the representative structure chosen.

A more complete representation of the results of the above current admixture procedure is given as the "exact" entries in table Al for various values of top-layer thickness, $t$, normalized to contact radius, $a$, and of the conductivity ratio, $\beta$. To derive the values in the table, after determining the admixture coefficients in each case for which $V(r=0)$ equals $V(r=0.9 a)$, the potential at $r=0$ was taken as the effective potential on the disk contact. An equal but opposite potential was assigned to the second contact. The reciprocal correction factor $\mathrm{H}$ was then determined for selected cases using appropriate admixtures of eqs (A3) and (A6) to calculate the resulting differences, $\Delta V$, of the potential between the probes, with $\mathrm{H}$ being given by: 


$$
H=\frac{\rho I}{2 a \Delta V} \text {. }
$$

The results for $\beta=1$ (corresponding to $\sigma_{2}=0^{\circ}$ ) are the most accurate values known for the isolated single layer correction factor and are therefore referred to as exact in this appendix. These results are used in section $A .4$ of this appendix to compare with the results of the SG assumptions on current distributions and are used below to calculate values for nonzero substrate conductivity using parallel superposition.

$$
\begin{aligned}
\text { Table AI - } & \text { Reciprocal Correction Factor, H, for a Single } \\
& \text { Layer of Thickness t on Lower-Conductivity, In- } \\
& \text { finite Substrate }
\end{aligned}
$$

\begin{tabular}{|c|c|c|c|c|c|}
\hline$t / a$ & 0 & 0.1 & 0.5 & 0.9 & 1.0 \\
\hline exact & 1.016173 & 0.874233 & 0.442556 & 0.134553 & 0.042562 \\
\hline approx & 1.016173 & 0.839153 & 0.367099 & 0.093805 & 0.042562 \\
\hline
\end{tabular}

$\beta$

\begin{tabular}{l|lllll} 
exact & 1.016173 & 0.963419 & 0.745523 & 0.478176 & 0.358947 \\
approx & 1.016173 & 0.898496 & 0.584689 & 0.403011 & 0.368947
\end{tabular}

$10 \mid \begin{array}{llllll}\text { exact } & 1.016173 & 1.012468 & 0.995510 & 0.972602 & 0.964729 \\ \text { approx } & 1.016173 & 1.006820 & 0.981877 & 0.967437 & 0.964729\end{array}$

To derive the results of parallel superposition analysis of the structure in figure Al for comparison with the foregoing, the reciprocal correction factor $H$ for measurements on the top of the structure is taken from eq (3):

$$
\mathrm{H}=\mathrm{G}_{1} / 2 \mathrm{a} \sigma_{1}
$$

Note that $\mathrm{H}$ is shown with no layer thickness dependence when it is the correction factor for the entire underlying structure. By parallel superposition analysis of the structure

$$
G=2 a\left[\left(\sigma_{1}-\sigma_{2}\right) H(t)+\sigma_{2} H(\infty)\right],
$$

where $H(t)$ and $H(\infty)$ are the values of $H$ appropriate to the two layers of the structure, see figure $A 1$. Then, combining eqs (A8) and (A9), the factor, $\mathrm{H}$, for the entire structure is given in terms of the individual $\mathrm{H}$ values for the two component layers by:

$$
H=\frac{\sigma_{1}-\sigma_{2}}{\sigma_{1}} H(t)+\frac{\sigma_{2}}{\sigma_{1}} H(\infty)
$$




$$
=\frac{2 \beta}{1+\beta} H(t)+\frac{(1-\beta)}{(1+\beta)} H(\infty) \text {. }
$$

Each component term, $\mathrm{H}(t)$ and $\mathrm{H}(\infty)$, is most accurately evaluated, in turn, as a case of the isolated single layer, using the current admixture formalism above. The factor $H$ from parallel superposition is thus evaluated for the same values of $t$ and $\beta$ and current admixture formalism as was the image-derived solution for $\mathrm{H}$ above; the results from parallel superposition are given as the "approx" entries in table Al.

Table Al compares values of the correction factor, $H$, derived from an image calculation, with values of $\mathrm{H}$ calculated by assuming that the structure could be represented as independent parallel conducting layers. Both calculations feature the same treatment of the boundary value potential under the contacts as determined from the generalized current distribution used in this work, and appropriate to the same infinite thickness of the top layer. The discrepancies seen in table Al between the results from parallel superposition and the "exact" results obtained by admixing eqs (A3) and (A6) are not serious, and yet they provide proof that the parallel superposition concept is not in general valid.

A.3 Exercises II and III - Parallel Superposition and Local Slope versus the Schumann and Gardner Boundary Value Solution

The model used for the following calculations consists of two uniform layers of differing conductivity on a third layer of still different conductivity. In Exercise II the third layer has infinite thickness, and in Exercise III the third layer has a thickness equal to that of the first two and is bounded below by an insulating surface. The structure is shown in figure A3. The SG method is used to calculate reference two-probe correction factors for the structures, and then the parallel superposition and the local slope methods are employed to calculate equivalent factors for comparison.

The correction factors calculated in the SG approach are found by numerical integration of the expression, [1]:

$$
F=\frac{4}{\pi} \int_{0}^{\infty}[1+2 \theta(k)]\left[\frac{J_{l}(k)}{k^{2}}-\frac{J_{0}(s k)}{2 k}\right] \sin (k) d k
$$

The integrals were evaluated using a trapezoid method with an interval $\mathrm{dk}=0.01$ and are truncated at the upper limit $\mathrm{k}=10 .^{*} \mathrm{~A}$ probe spacing,

* An interval $\mathrm{dk}=0.005$ and an upper limit $\mathrm{k}=20$ were used for several integrations, with insignificant change in the results. 


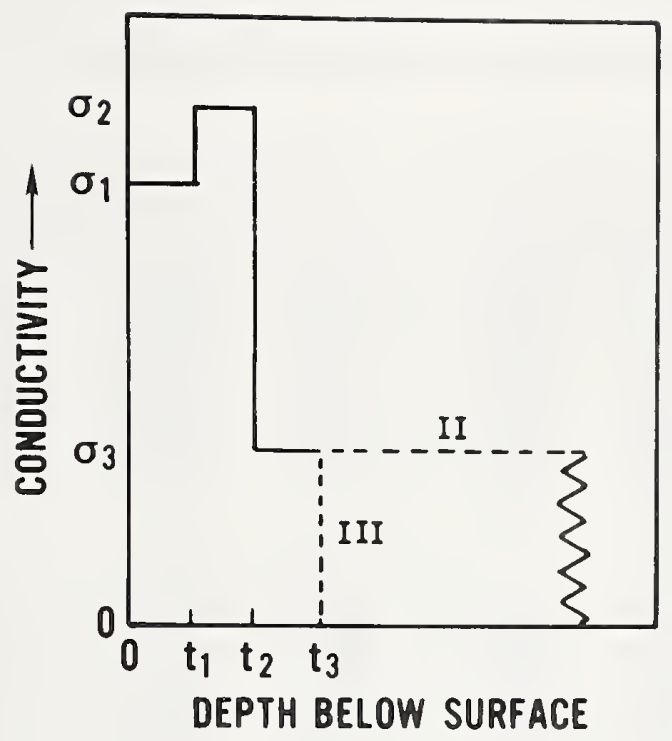

Figure A3. Schematic conductivity profile for Computer Exercises II and III. The layer with conductivity $\sigma_{3}$ is infinitely thick for Exercise II.

s, equal to 40 contact radii was assumed for all the calculations. The integration factor for the first structure (Exercise II) is

$$
1+2(K)=\frac{1+\beta_{1} E_{1}+\beta_{2} E_{2}+\beta_{1} \beta_{2} E_{2} / E_{1}}{1-\beta_{1} E_{1}-\beta_{2} E_{2}+\beta_{1} \beta_{2} E_{2} / E_{1}}
$$

and for the second structure, it is

$1+2 \theta(K)=$

$$
\frac{\left(1+E_{3}\right) E_{1} E_{2}+\beta_{1} E_{2}\left(E_{3}+E_{1}^{2}\right)+\beta_{2} E_{1}\left(E_{3}+E_{2}{ }^{2}\right)+\beta_{1} \beta_{2}\left(E_{2}{ }^{2}+E_{1}{ }^{2} E_{3}\right)}{\left(1-E_{3}\right) E_{1} E_{2}+\beta_{1} E_{2}\left(E_{3}-E_{1}{ }^{2}\right)+\beta_{2} E_{1}\left(E_{3}-E_{2}{ }^{2}\right)+\beta_{1} \beta_{2}\left(E_{2}{ }^{2}-E_{1}{ }^{2} E_{3}\right)}
$$

where

$$
E_{i}=e^{-2 k t_{i}}
$$

and

$$
\beta_{i}=\frac{\left(\sigma_{i}-\sigma_{i+1}\right)}{\left(\sigma_{i}+\sigma_{i+1}\right)} \text {. }
$$

The correction factors obtained from the SG technique for the various combinations of thickness and conductivity ratios are listed in table A2 for the first structure and in table A3 for the second structure. These 
Table A2 - Sampling Volume Correction Factors for Exercise II*. $\beta_{1}$

\begin{tabular}{|c|c|c|c|c|c|c|c|c|}
\hline & $\mathrm{B}_{2}$ & 0.9 & 0.6 & 0.3 & 0.0 & -0.3 & -0.6 & -0.9 \\
\hline \multirow{3}{*}{$t_{1}=0.05 a$} & 0.9 & 34.532 & 19.355 & 12.401 & 8.008 & 4.878 & 2.495 & 0.601 \\
\hline & 0.6 & 21.488 & 8.875 & 4.976 & 2.959 & 1.708 & 0.851 & 0.225 \\
\hline & 0.3 & 15.078 & 5.298 & 2.805 & 1.622 & 0.927 & 0.467 & 0.141 \\
\hline \multirow[t]{2}{*}{$t_{2}=0.10 a$} & 0.0 & 10.669 & 3.326 & 1.704 & 0.976 & 0.560 & 0.291 & 0.103 \\
\hline & -0.3 & 7.210 & 2.042 & 1.029 & 0.591 & 0.346 & 0.190 & 0.081 \\
\hline \multirow{2}{*}{$t_{3}=\infty$} & -0.6 & 4.286 & 1.128 & 0.570 & 0.335 & 0.206 & 0.124 & 0.067 \\
\hline & -0.9 & 1.683 & 0.438 & 0.236 & 0.152 & 0.106 & 0.077 & 0.057 \\
\hline \multirow{3}{*}{$t_{1}=0.10 a$} & 0.9 & 19.499 & 12.277 & 8.326 & 5.601 & 3.533 & 1.879 & 0.509 \\
\hline & 0.6 & 13.846 & 6.652 & 3.996 & 2.493 & 1.502 & 0.791 & 0.253 \\
\hline & 0.3 & 10.525 & $4 \cdot 360$ & 2.474 & 1.502 & 0.899 & 0.486 & 0.185 \\
\hline \multirow[t]{2}{*}{$t_{2}=0.20 a$} & 0.0 & 8.008 & 2.959 & 1.622 & 0.976 & 0.591 & 0.335 & 0.152 \\
\hline & -0.3 & 5.866 & 1.970 & 1.062 & 0.644 & 0.402 & 0.244 & 0.132 \\
\hline \multirow[t]{2}{*}{$t_{3}=\infty$} & -0.6 & 3.901 & 1.217 & 0.659 & 0.412 & 0.272 & 0.182 & 0.119 \\
\hline & -0.9 & 1.990 & 0.614 & 0.354 & 0.241 & 0.178 & 0.138 & 0.109 \\
\hline \multirow{3}{*}{$t_{1}=0.30 a$} & 0.9 & 7.335 & 5.372 & 3.980 & 2.881 & 1.966 & 1.179 & 0.487 \\
\hline & 0.6 & 6.157 & 3.703 & 2.513 & 1.738 & 1.173 & 0.734 & 0.379 \\
\hline & 0.3 & 5.237 & 2.820 & 1.846 & 1.265 & 0.866 & 0.571 & 0.340 \\
\hline \multirow[t]{2}{*}{$t_{2}=0.60 a$} & 0.0 & 4.424 & 2.202 & 1.417 & 0.976 & 0.686 & 0.477 & 0.319 \\
\hline & -0.3 & 3.650 & 1.717 & 1.102 & 0.773 & 0.563 & 0.415 & 0.305 \\
\hline \multirow[t]{2}{*}{$t_{3}=\infty$} & -0.6 & 2.872 & 1.310 & 0.854 & 0.619 & 0.472 & 0.370 & 0.295 \\
\hline & -0.9 & 2.045 & 0.955 & 0.651 & 0.496 & 0.401 & 0.335 & 0.288 \\
\hline \multirow{3}{*}{$t_{1}=1.2 a$} & 0.9 & 2.245 & 1.918 & 1.633 & 1.376 & 1.143 & 0.927 & 0.727 \\
\hline & 0.6 & 2.137 & 1.690 & 1.394 & 1.170 & 0.988 & 0.835 & 0.703 \\
\hline & 0.3 & 2.025 & 1.523 & 1.247 & 1.055 & 0.909 & 0.791 & 0.693 \\
\hline \multirow[t]{2}{*}{$t_{2}=2.4 a$} & 0.0 & 1.906 & 1.386 & 1.138 & 0.976 & 0.857 & 0.763 & 0.686 \\
\hline & -0.3 & 1.774 & 1.267 & 1.051 & 0.916 & 0.818 & 0.743 & 0.681 \\
\hline \multirow{2}{*}{$t_{3}=\infty$} & -0.6 & 1.623 & 1.160 & 0.978 & 0.867 & 0.788 & 0.727 & 0.678 \\
\hline & -0.9 & 1.444 & 1.061 & 0.915 & 0.826 & 0.763 & 0.714 & 0.675 \\
\hline \multirow{3}{*}{$t_{1}=5 a$} & 0.9 & 1.138 & 1.097 & 1.058 & 1.020 & 0.984 & 0.949 & 0.915 \\
\hline & 0.6 & 1.132 & 1.081 & 1.039 & 1.002 & 0.969 & 0.940 & 0.913 \\
\hline & 0.3 & 1.126 & 1.066 & 1.023 & 0.988 & 0.958 & 0.933 & 0.911 \\
\hline \multirow[t]{2}{*}{$t_{2}=10 a$} & 0.0 & 1.118 & 1.052 & 1.009 & 0.976 & 0.950 & 0.929 & 0.910 \\
\hline & -0.3 & 1.108 & 1.037 & 0.996 & 0.966 & 0.943 & 0.925 & 0.909 \\
\hline \multirow[t]{2}{*}{$t_{3}=\infty$} & -0.6 & 1.095 & 1.023 & 0.984 & 0.958 & 0.938 & 0.922 & 0.908 \\
\hline & -0.9 & 1.081 & 1.009 & 0.973 & 0.950 & 0.933 & 0.919 & 0.908 \\
\hline
\end{tabular}

* Obtained using the method of Schumann and Gardner for two uniform layers on an infinite substrate. 
Table A3- Sampling Volume Correction Factors for Exercise III*.

$\beta_{1}$

\begin{tabular}{|c|c|c|c|c|c|c|c|c|}
\hline & $\mathrm{B}_{2}$ & 0.9 & 0.6 & 0.3 & 0.0 & -0.3 & -0.6 & -0.9 \\
\hline \multirow{3}{*}{$t_{1}=0.05 a$} & 0.9 & 46.483 & 38.847 & 31.329 & 23.926 & 16.636 & 9.457 & 2.385 \\
\hline & 0.6 & 46.031 & 37.390 & $29 \cdot 344$ & 21.834 & 14.808 & 8.221 & 2.032 \\
\hline & 0.3 & 45.387 & 35.449 & 26.860 & 19.363 & 12.762 & 6.905 & 1.674 \\
\hline \multirow[t]{2}{*}{$t_{2}=0.10 a$} & 0.0 & 44.393 & 32.732 & 23.659 & 16.398 & 10.455 & 5.502 & 1.309 \\
\hline & -0.3 & 42.660 & 28.660 & 19.378 & 12.774 & 7.835 & 4.001 & 0.940 \\
\hline \multirow{2}{*}{$t_{3}=0.15 a$} & -0.6 & 38.878 & 21.880 & $13 \cdot 363$ & 8.247 & 4.834 & 2.394 & 0.564 \\
\hline & -0.9 & $24 \cdot 142$ & 8.347 & 4.289 & 2.428 & 1.361 & 0.668 & 0.183 \\
\hline \multirow{3}{*}{$t_{1}=0.10 \mathrm{a}$} & 0.9 & 23.270 & 19.460 & 15.709 & 12.015 & 8.378 & 4.795 & 1.266 \\
\hline & 0.6 & 23.046 & 18.736 & 14.722 & 10.975 & 7.469 & $4 \cdot 180$ & 1.091 \\
\hline & 0.3 & 22.725 & 17.772 & 13.488 & 9.746 & 6.451 & 3.526 & 0.912 \\
\hline \multirow{2}{*}{$t_{2}=0.20 a$} & 0.0 & 22.233 & 16.424 & 11.898 & 8.273 & 5.304 & 2.828 & 0.731 \\
\hline & -0.3 & 21.376 & 14.407 & 9.775 & 6.475 & 4.003 & 2.082 & 0.547 \\
\hline \multirow{2}{*}{$t_{3}=0.30 a$} & -0.6 & $19 \cdot 516$ & 11.058 & 6.796 & 4.229 & 2.512 & 1.283 & 0.360 \\
\hline & -0.9 & 12.373 & 4.404 & 2.312 & 1.346 & 0.788 & 0.426 & 0.171 \\
\hline \multirow{3}{*}{$t_{1}=0.30 \mathrm{a}$} & 0.9 & 7.854 & 6.609 & 5.382 & $4 \cdot 174$ & 2.984 & 1.811 & 0.656 \\
\hline & 0.6 & 7.783 & 6.378 & 5.068 & 3.842 & 2.694 & 1.615 & 0.600 \\
\hline & 0.3 & 7.682 & 6.073 & 4.676 & 3.452 & 2.370 & 1.406 & 0.543 \\
\hline \multirow{2}{*}{$t_{2}=0.60 a$} & 0.0 & 7.528 & 5.650 & 4.175 & 2.986 & 2.006 & 1.184 & 0.485 \\
\hline & -0.3 & 7.264 & 5.024 & 3.511 & 2.419 & 1.593 & 0.947 & 0.426 \\
\hline \multirow{2}{*}{$t_{3}=0.90 a$} & -0.6 & 6.707 & 3.999 & 2.586 & 1.715 & 1.123 & 0.693 & 0.366 \\
\hline & -0.9 & 4.581 & 2.008 & 1.211 & 0.817 & 0.580 & 0.421 & 0.306 \\
\hline \multirow{3}{*}{$t_{1}=1.2 a$} & 0.9 & 2.278 & 2.014 & 1.753 & 1.496 & 1.242 & 0.992 & 0.745 \\
\hline & 0.6 & 2.265 & 1.970 & 1.693 & 1.433 & 1.187 & 0.954 & 0.734 \\
\hline & 0.3 & 2.246 & 1.913 & 1.620 & 1.359 & 1.125 & 0.914 & 0.723 \\
\hline \multirow[t]{2}{*}{$t_{2}=2.4 a$} & 0.0 & 2.218 & 1.836 & 1.527 & 1.272 & 1.057 & 0.872 & 0.712 \\
\hline & -0.3 & 2.172 & 1.724 & 1.406 & 1.167 & 0.979 & 0.827 & 0.701 \\
\hline \multirow{2}{*}{$t_{3}=3.6 a$} & -0.6 & 2.079 & 1.546 & 1.241 & 1.039 & 0.892 & 0.779 & 0.689 \\
\hline & -0.9 & 1.772 & 1.215 & 1.002 & 0.877 & 0.792 & 0.728 & 0.678 \\
\hline \multirow{3}{*}{$t_{1}=5 a$} & 0.9 & 1.140 & 1.101 & 1.063 & 1.026 & 0.989 & 0.953 & 0.916 \\
\hline & 0.6 & 1.138 & 1.096 & 1.056 & 1.018 & 0.982 & 0.948 & 0.915 \\
\hline & 0.3 & 1.136 & 1.089 & 1.047 & 1.009 & 0.975 & 0.943 & 0.914 \\
\hline \multirow{2}{*}{$t_{2}=10 a$} & 0.0 & 1.133 & 1.080 & 1.037 & 0.999 & 0.967 & 0.938 & 0.912 \\
\hline & -0.3 & 1.128 & 1.068 & 1.023 & 0.987 & 0.957 & 0.932 & 0.911 \\
\hline \multirow{2}{*}{$t_{3}=15 a$} & -0.6 & 1.119 & 1.050 & 1.005 & 0.972 & 0.947 & 0.927 & 0.910 \\
\hline & -0.9 & 1.097 & 1.020 & 0.981 & 0.955 & 0.936 & 0.921 & 0.908 \\
\hline
\end{tabular}

* Obtained using the method of Schumann and Gardner for three uniform layers on an insulating substrate. 
factors are taken as a point of reference and assumed to be accurate; the factors calculated using the parallel superposition and local slope approaches are compared with them in succeeding tables.

For the parallel superposition calculations, eq (8) is combined with eq (A8) to derive an expression for $\mathrm{F}$, the reciprocal of $\mathrm{H}$, for the entire structure:

$$
F=\frac{1}{H}=\frac{1}{\sum_{i}\left[\sigma_{i} / \sigma_{1}-\sigma_{i+1} / \sigma_{1}\right] H\left(t_{i}\right)}
$$

where the terms $\mathrm{H}\left(t_{j}\right)$ are those appropriate to component sublayers of thickness $t_{i}$. In contrast to Exercise $I$ where the sublayer $H(t)$ values were evaluated from the current admixture formalism, eq (A10) ff., here they are evaluated from the SG formalism applied to single isolated layers of thickness $t_{i}$. The results of the calculations based on eq (Al6) are listed for the two structures in cables A4 and A5. They are shown there, for each combination of thickness and conductivity ratio, as the ratio of $F$ derived from parallel superposition, eq (A16), to the $F$ derived directly from the SG approach, using eqs (Al2) or (Al3), as appropriate. Note that for all tables (A2 to A7) in Exercises II and III the values for $t_{1}, t_{2}, t_{3}$ as listed are cumulative thickness values, in keeping with the formalism of SG [1], and not single layer thickness values.

Comparisons of the results of local slope calculations for $F$ with the SG values for $F$ on the same two structures are given in tables $A 6$ and A7. To generate these results, one needs to simulate real data so as to calculate local slopes. To do this, one calculates "true" factors (using SG) for values of $t$ slightly smaller and larger than nominal. For example, in the case for $t=0.1 a$, SG factors have also been generated using $t_{1}=0.09 \mathrm{a}$ and $t_{1}={ }^{1} 0.1 \mathrm{a}$ (keeping $t_{2}=0.2 \mathrm{a}$ and $t_{3}=0.3 \mathrm{a}$ or $\infty$, as appropriate). The "local slope" is then tound from

$$
m=\frac{d(\log F)}{d t}=\frac{\log F\left(t_{1}=0.1 \operatorname{la}\right)-\log F\left(t_{1}=0.09 a\right)}{0.02 a} .
$$

Having defined a profile slope for each case, one uses eq (22) to calculate correction factors for the two structures. The substrate factor, $\mathrm{K}_{1} \mathrm{~K}_{2}$, in eq (22) is replaced by unity for the purpose of these calculations. * This modification should improve the comparison based on eq (22) in the vicinity of $\beta_{1}=\beta_{2}=0$ where the structure resembles a large uniform slab, but it simultaneously causes poorer comparison with SG in the direction of lower values for $\mathrm{F}$ (from local slope) for cases of $\beta_{1}$ and $\beta_{2}$ ' both negative, where the structure resembles the conducting substrate limit.

* The substrate factor given in eq (24) was developed subsequent to the generation of tables $\mathrm{A} 6$ and $\mathrm{A} 7$. 
Table A4 - The Ratio of Correction Factor from Parallel Superposition to that from Schumann and Gardner for Exercise II*.

$\beta_{1}$

\begin{tabular}{|c|c|c|c|c|c|c|c|c|}
\hline & $\mathrm{B}_{2}$ & 0.9 & 0.6 & 0.3 & 0.0 & -0.3 & -0.6 & -0.9 \\
\hline \multirow{3}{*}{$t_{1}=0.05 a$} & 0.9 & 1.153 & 1.307 & 1.332 & 1.325 & 1.308 & 1.280 & 1.182 \\
\hline & 0.6 & 1.318 & 1.281 & 1.219 & 1.173 & 1.133 & 1.080 & 0.874 \\
\hline & 0.3 & 1.320 & 1.189 & 1.123 & 1.079 & 1.035 & 0.964 & 0.679 \\
\hline \multirow{2}{*}{$t_{2}=0.10 a$} & 0.0 & 1.265 & 1.105 & 1.045 & 1.000 & 0.947 & 0.851 & 0.510 \\
\hline & -0.3 & 1.172 & 1.015 & 0.957 & 0.906 & 0.837 & 0.712 & 0.352 \\
\hline \multirow{2}{*}{$t_{3}=\infty$} & -0.6 & 1.018 & 0.879 & 0.817 & 0.751 & 0.661 & 0.511 & 0.199 \\
\hline & -0.9 & 0.592 & 0.486 & 0.421 & 0.352 & 0.272 & 0.174 & 0.049 \\
\hline \multirow{3}{*}{$t_{1}=0.10 a$} & 0.9 & 1.082 & 1.244 & 1.307 & 1.330 & 1.330 & 1.301 & 1.101 \\
\hline & 0.6 & 1.264 & 1.344 & 1.305 & 1.255 & 1.195 & 1.098 & 0.743 \\
\hline & 0.3 & 1.331 & 1.277 & 1.197 & 1.127 & 1.049 & 0.920 & 0.517 \\
\hline \multirow{2}{*}{$t_{2}=0.20 \mathrm{a}$} & 0.0 & 1.325 & 1.173 & 1.079 & 1.000 & 0.906 & 0.751 & 0.352 \\
\hline & -0.3 & 1.248 & 1.037 & 0.937 & 0.849 & 0.740 & 0.571 & 0.223 \\
\hline \multirow{2}{*}{$t_{3}=\infty$} & -0.6 & 1.057 & 0.828 & 0.726 & 0.631 & 0.517 & 0.360 & 0.117 \\
\hline & -0.9 & 0.510 & 0.360 & 0.292 & 0.231 & 0.169 & 0.101 & 0.027 \\
\hline \multirow{3}{*}{$t_{1}=0.30 a$} & 0.9 & 1.024 & 1.136 & 1.203 & 1.237 & 1.229 & 1.135 & 0.666 \\
\hline & 0.6 & 1.149 & 1.333 & 1.362 & 1.321 & 1.215 & 0.997 & 0.438 \\
\hline & 0.3 & 1.246 & 1.368 & 1.308 & 1.194 & 1.028 & 0.771 & 0.284 \\
\hline \multirow{2}{*}{$t_{2}=0.60 \mathrm{a}$} & 0.0 & 1.312 & 1.298 & 1.158 & 1.000 & 0.812 & 0.563 & 0.182 \\
\hline & -0.3 & 1.319 & 1.124 & 0.934 & 0.762 & 0.584 & 0.375 & 0.109 \\
\hline \multirow[t]{2}{*}{$t_{3}=\infty$} & -0.6 & 1.175 & 0.813 & 0.624 & 0.478 & 0.344 & 0.206 & 0.055 \\
\hline & -0.9 & 0.534 & 0.270 & 0.187 & 0.133 & 0.089 & 0.049 & 0.012 \\
\hline \multirow{3}{*}{$t_{1}=1.2 a$} & 0.9 & 1.019 & 1.070 & 1.093 & 1.073 & 0.978 & 0.750 & 0.263 \\
\hline & 0.6 & 1.061 & 1.171 & 1.197 & 1.141 & 0.984 & 0.693 & 0.215 \\
\hline & 0.3 & 1.106 & 1.235 & 1.222 & 1.108 & 0.898 & 0.588 & 0.167 \\
\hline \multirow[t]{2}{*}{$t_{2}=2.4 \mathrm{a}$} & 0.0 & 1.152 & 1.257 & 1.176 & 1.000 & 0.758 & 0.464 & 0.123 \\
\hline & -0.3 & 1.195 & 1.210 & 1.038 & 0.816 & 0.576 & 0.330 & 0.082 \\
\hline \multirow{2}{*}{$t_{3}=\infty$} & -0.6 & 1.201 & 1.016 & 0.763 & 0.543 & 0.354 & 0.190 & 0.045 \\
\hline & -0.9 & 0.863 & 0.424 & 0.254 & 0.159 & 0.095 & 0.048 & 0.011 \\
\hline \multirow{3}{*}{$t_{1}=5 a$} & 0.9 & 1.021 & 1.032 & 1.033 & 1.013 & 0.955 & 0.807 & 0.364 \\
\hline & 0.6 & 1.025 & 1.044 & 1.045 & 1.019 & 0.950 & 0.786 & 0.340 \\
\hline & 0.3 & 1.030 & 1.054 & 1.050 & 1.016 & 0.933 & 0.753 & 0.309 \\
\hline \multirow[t]{2}{*}{$t_{2}=10 a$} & 0.0 & 1.036 & 1.060 & 1.048 & 1.000 & 0.900 & 0.702 & 0.269 \\
\hline & -0.3 & 1.042 & 1.059 & 1.031 & 0.962 & 0.837 & 0.621 & 0.217 \\
\hline \multirow{2}{*}{$t_{3}=\infty$} & -0.6 & 1.045 & 1.037 & 0.974 & 0.866 & 0.708 & 0.481 & 0.147 \\
\hline & -0.9 & 1.005 & 0.849 & 0.672 & 0.499 & 0.337 & 0.185 & 0.045 \\
\hline
\end{tabular}

* Two uniform layers on an infinite substrate. 
Table A5 - The Ratio of Correction Factor from Parallel superposition to that from Schumann and Gardner for Exercise III*.

$\beta_{1}$

\begin{tabular}{l|ccccccc}
\hline$\beta_{2}$ & 0.9 & 0.6 & 0.3 & 0.0 & -0.3 & -0.6 & -0.9 \\
\hline 0.9 & 1.000 & 1.000 & 1.000 & 1.000 & 0.999 & 0.997 & 0.981 \\
0.6 & 1.000 & 1.000 & 1.000 & 1.000 & 0.999 & 0.997 & 0.978 \\
0.3 & 1.000 & 1.000 & 1.000 & 1.000 & 0.999 & 0.996 & 0.973 \\
0.0 & 1.000 & 1.001 & 1.001 & 1.000 & 0.999 & 0.994 & 0.964 \\
-0.3 & 1.000 & 1.000 & 1.000 & 0.999 & 0.997 & 0.991 & 0.949 \\
-0.6 & 1.000 & 0.999 & 0.998 & 0.996 & 0.992 & 0.981 & 0.911 \\
-0.9 & 0.993 & 0.984 & 0.977 & 0.968 & 0.951 & 0.912 & 0.703
\end{tabular}

\begin{tabular}{|c|c|c|c|c|c|c|c|c|}
\hline & 0.9 & 1.000 & 0.998 & 0.997 & 0.994 & 0.990 & 0.981 & 0.921 \\
\hline$t_{1}=0.10 \mathrm{a}$ & 0.6 & 1.000 & 0.999 & 0.998 & 0.996 & 0.992 & 0.982 & 0.913 \\
\hline & 0.3 & 1.000 & 1.001 & 1.000 & 0.998 & 0.994 & 0.982 & 0.899 \\
\hline$t_{2}=0.20 a$ & 0.0 & 1.001 & 1.002 & 1.002 & 1.000 & 0.995 & 0.979 & 0.875 \\
\hline & -0.3 & 1.001 & 1.003 & 1.003 & 1.000 & $0.99 \hat{1}$ & 0.969 & 0.83 \\
\hline$t_{3}=0.30 a$ & $\begin{array}{l}-0.6 \\
-0.9\end{array}$ & $\begin{array}{l}1.002 \\
0.984\end{array}$ & $\begin{array}{l}1.003 \\
0.957\end{array}$ & $\begin{array}{l}0.999 \\
0.932\end{array}$ & $\begin{array}{l}0.991 \\
0.898\end{array}$ & $\begin{array}{l}0.975 \\
0.845\end{array}$ & $\begin{array}{l}0.936 \\
0.738\end{array}$ & $\begin{array}{l}0.730 \\
0.391\end{array}$ \\
\hline
\end{tabular}

\begin{tabular}{|c|c|c|c|c|c|c|c|c|}
\hline & 0.9 & 1.001 & 1.004 & 1.004 & 0.998 & 0.980 & 0.924 & 0.639 \\
\hline$t_{1}=0.30 a$ & 0.6 & 1.002 & 1.008 & 1.008 & 1.002 & 0.980 & 0.915 & 0.604 \\
\hline & 0.3 & 1.004 & 1.011 & 1.012 & 1.003 & 0.976 & 0.898 & 0.557 \\
\hline$t_{2}=0.60 a$ & 0.0 & 1.005 & 1.015 & 1.014 & 1.000 & 0.962 & 0.864 & 0.492 \\
\hline & -0.3 & 1.007 & 1.016 & 1.009 & 0.982 & 0.926 & 0.800 & 0.403 \\
\hline$=0.90 a$ & -0.6 & 1.008 & 1.002 & 0.972 & 0.917 & 0.827 & 0.662 & 0.27 \\
\hline & -0.9 & 0.941 & 0.796 & 0.684 & 0.572 & 0.446 & 0.290 & 0.0 \\
\hline
\end{tabular}

\begin{tabular}{|c|c|c|c|c|c|c|c|c|}
\hline \multirow{3}{*}{$t_{1}=1.2 \mathrm{a}$} & 0.9 & 1.008 & 1.025 & 1.025 & 0.996 & 0.911 & 0.712 & 0.261 \\
\hline & 0.6 & 1.011 & 1.035 & 1.038 & 1.004 & 0.908 & 0.693 & 0.243 \\
\hline & 0.3 & 1.015 & 1.047 & 1.050 & 1.008 & 0.894 & 0.662 & 0.221 \\
\hline \multirow{2}{*}{$t_{2}=2.4 a$} & 0.0 & 1.021 & 1.061 & 1.059 & 1.000 & 0.862 & 0.611 & 0.191 \\
\hline & -0.3 & 1.030 & 1.075 & 1.054 & 0.962 & 0.790 & 0.527 & 0.153 \\
\hline \multirow[t]{2}{*}{$t_{3}=3.6 a$} & -0.6 & 1.044 & 1.070 & 0.988 & 0.836 & 0.631 & 0.384 & 0.1 .02 \\
\hline & -0.9 & 1.013 & 0.775 & 0.555 & 0.383 & 0.244 & 0.129 & 0.030 \\
\hline \multirow{3}{*}{$t_{1}=5 \mathrm{a}$} & 0.9 & 1.006 & 1.020 & 1.025 & 1.016 & 0.974 & 0.851 & 0.419 \\
\hline & 0.6 & 1.007 & 1.022 & 1.027 & 1.015 & 0.966 & 0.832 & 0.394 \\
\hline & 0.3 & 1.008 & 1.025 & 1.028 & 1.010 & 0.952 & 0.804 & 0.363 \\
\hline \multirow{2}{*}{$t_{2}=10 \mathrm{a}$} & 0.0 & 1.009 & 1.027 & 1.027 & 1.000 & 0.928 & 0.761 & 0.323 \\
\hline & -0.3 & 1.011 & 1.028 & 1.019 & 0.976 & 0.881 & 0.691 & 0.267 \\
\hline \multirow[t]{2}{*}{$t_{3}=15 a$} & -0.6 & 1.013 & 1.020 & 0.985 & 0.908 & 0.776 & 0.560 & 0.186 \\
\hline & -0.9 & 0.995 & 0.893 & 0.747 & 0.585 & 0.414 & 0.238 & 0.060 \\
\hline
\end{tabular}

Three uniform layers on an insulating substrate. 
Table A6 - The Ratio of Correction Factor from the Local Slope Method to that from Schumann and Gardner for Exercise II*.

$\beta_{1}$

\begin{tabular}{r|cllllll}
\hline$B_{2}$ & 0.9 & 0.6 & 0.3 & 0.0 & -0.3 & -0.6 & -0.9 \\
\hline 0.9 & 1.031 & 1.197 & 1.308 & 1.384 & 1.416 & 1.302 & 0.509 \\
0.6 & 1.234 & 1.577 & 1.711 & 1.709 & 1.447 & 0.737 & 0.435 \\
0.3 & 1.414 & 1.795 & 1.830 & 1.551 & 0.888 & 0.513 & 0.425 \\
0.0 & 1.595 & 1.931 & 1.707 & 1.000 & 0.579 & 0.458 & 0.420 \\
-0.3 & 1.804 & 1.945 & 1.204 & 0.629 & 0.483 & 0.436 & 0.417 \\
-0.6 & 2.105 & 1.573 & 0.662 & 0.493 & 0.445 & 0.426 & 0.415 \\
-0.9 & 2.921 & 0.683 & 0.484 & 0.443 & 0.428 & 0.419 & 0.413
\end{tabular}

\begin{tabular}{|c|c|c|c|c|c|c|c|c|}
\hline \multirow{3}{*}{$t_{1}=0.10 \mathrm{a}$} & 0.9 & 0.986 & 1.082 & 1.156 & 1.202 & 1.200 & 1.021 & 0.494 \\
\hline & 0.6 & 1.107 & 1.368 & 1.478 & 1.459 & 1.203 & 0.684 & 0.457 \\
\hline & 0.3 & 1.237 & 1.563 & 1.601 & 1.362 & 0.851 & 0.542 & 0.448 \\
\hline \multirow{2}{*}{$t_{2}=0.20 a$} & 0.0 & 1.384 & 1.709 & 1.551 & 1.000 & 0.629 & 0.493 & 0.443 \\
\hline & -0.3 & 1.570 & 1.787 & 1.257 & 0.717 & 0.534 & 0.469 & 0.440 \\
\hline \multirow{2}{*}{$t_{3}=\infty$} & -0.6 & 1.861 & 1.679 & 0.830 & 0.569 & 0.490 & 0.456 & 0.438 \\
\hline & -0.9 & 2.631 & 1.081 & 0.595 & 0.502 & 0.467 & 0.448 & 0.437 \\
\hline
\end{tabular}

\begin{tabular}{|c|c|c|c|c|c|c|c|c|}
\hline \multirow{3}{*}{$t_{1}=0.30 \mathrm{a}$} & 0.9 & 0.943 & 0.965 & 0.979 & 0.972 & 0.916 & 0.766 & 0.585 \\
\hline & 0.6 & 0.981 & 1.091 & 1.131 & 1.086 & 0.931 & 0.719 & 0.577 \\
\hline & 0.3 & 1.036 & 1.205 & 1.217 & 1.087 & 0.863 & 0.676 & 0.571 \\
\hline \multirow[t]{2}{*}{$t_{2}=0.60 a$} & 0.0 & 1.110 & 1.310 & 1.249 & 1.000 & 0.791 & 0.645 & 0.567 \\
\hline & -0. & 1.216 & 1.410 & 1.227 & 0.935 & 0.733 & 0.624 & 0.564 \\
\hline \multirow{2}{*}{$t_{3}=\infty$} & -0.6 & 1.392 & 1.504 & 1.151 & 0.847 & 0.689 & 0.608 & 0.561 \\
\hline & -0.9 & 1.777 & 1.579 & 1.036 & 0.773 & 0.657 & 0.596 & 0.559 \\
\hline
\end{tabular}

\begin{tabular}{|c|c|c|c|c|c|c|c|c|}
\hline & 0.9 & 0.844 & 0.847 & 0.856 & 0.874 & 0.902 & 0.943 & 1.003 \\
\hline$t_{1}=1.2 \mathrm{a}$ & 0.6 & 0.850 & 0.879 & 0.910 & 0.940 & 0.968 & 0.995 & 1.020 \\
\hline & 0.3 & 0.861 & 0.917 & 0.958 & 0.987 & 1.007 & 1.020 & 1.027 \\
\hline$t_{2}=2.4 a$ & 0.0 & 0.877 & 0.958 & 1.001 & 1.000 & 1.034 & 1.036 & 1.031 \\
\hline & -0.3 & 0.903 & 1.003 & 1.042 & 1.055 & 1.055 & 1.046 & 1.033 \\
\hline$t_{3}=\infty$ & -0.6 & 0.345 & 1.054 & 1.081 & 1.081 & 1.070 & 1.054 & 1.035 \\
\hline & -0.9 & 1.019 & 1.113 & 1.119 & 1.104 & 1.083 & 1.059 & 1.036 \\
\hline
\end{tabular}

\begin{tabular}{|c|c|c|c|c|c|c|c|c|}
\hline \multirow{3}{*}{$t_{1}=5 a$} & 0.9 & 0.926 & 0.945 & 0.966 & 0.989 & 1.014 & 1.042 & 1.071 \\
\hline & 0.6 & 0.929 & 0.955 & 0.979 & 1.004 & 1.027 & 1.051 & 1.074 \\
\hline & 0.3 & 0.932 & 0.964 & 0.991 & 1.015 & 1.037 & 1.057 & 1.075 \\
\hline \multirow{2}{*}{$t_{2}=10 a$} & 0.0 & 0.936 & 0.974 & 1.002 & 1.000 & 1.044 & 1.061 & 1.076 \\
\hline & -0.3 & 0.942 & 0.984 & 1.012 & 1.033 & 1.050 & 1.065 & 1.077 \\
\hline \multirow{2}{*}{$t_{3}=\infty$} & -0.6 & 0.949 & 0.994 & 1.021 & 1.040 & 1.055 & 1.068 & 1.078 \\
\hline & -0 . & 0.959 & 1.005 & 1.030 & 1.047 & 1.060 & 1.070 & 1.079 \\
\hline
\end{tabular}

* Two uniform layers on an infinite substrate. 
Table A7 - The Ratio of Correction Factor from the Local slope Method to that from Schumann and Gardner for Exercise III*.

$\beta_{1}$

\begin{tabular}{c|ccccccc}
\hline$B_{2}$ & 0.9 & 0.6 & 0.3 & 0.0 & -0.3 & -0.6 & -0.9 \\
\hline 0.9 & 0.969 & 0.965 & 0.962 & 0.958 & 0.954 & 0.943 & 0.765 \\
0.6 & 0.969 & 0.964 & 0.961 & 0.957 & 0.953 & 0.938 & 0.715 \\
0.3 & 0.968 & 0.963 & 0.960 & 0.955 & 0.950 & 0.930 & 0.650 \\
0.0 & 0.968 & 0.962 & 0.958 & 0.954 & 0.946 & 0.915 & 0.574 \\
-0.3 & 0.967 & 0.951 & 0.956 & 0.950 & 0.937 & 0.879 & 0.501 \\
-0.6 & 0.965 & 0.958 & 0.951 & 0.939 & 0.903 & 0.768 & 0.447 \\
-0.9 & 0.959 & 0.943 & 0.896 & 0.781 & 0.593 & 0.463 & 0.420
\end{tabular}

\begin{tabular}{|c|c|c|c|c|c|c|c|c|}
\hline & 0.9 & 0.958 & 0.955 & 0.954 & 0.949 & 0.940 & 0.905 & 0.585 \\
\hline$=0.10 a$ & 0.6 & 0.958 & 0.956 & 0.953 & 0.948 & 0.935 & 0.889 & 0.549 \\
\hline & 0.3 & 0.958 & 0.955 & 0.952 & 0.945 & 0.928 & 0.865 & 0.517 \\
\hline $0.20 \mathrm{a}$ & 0.0 & 0.958 & 0.954 & 0.949 & 0.940 & 0.915 & 0.822 & 0.488 \\
\hline & -0.3 & 0.957 & 0.953 & 0.945 & 0.929 & 0.886 & 0.742 & 0.465 \\
\hline$=0.30 \mathrm{a}$ & -0.6 & 0.955 & 0.949 & 0.934 & 0.896 & 0.800 & 0.597 & 0.449 \\
\hline & -0.9 & 0.955 & 0.925 & 0.818 & 0.646 & 0.520 & 0.462 & 0.439 \\
\hline
\end{tabular}

\begin{tabular}{|c|c|c|c|c|c|c|c|c|}
\hline \multirow{3}{*}{$t_{1}=0.30 a$} & 0.9 & 0.938 & 0.931 & 0.920 & 0.900 & 0.859 & 0.760 & 0.582 \\
\hline & 0.6 & 0.938 & 0.930 & 0.917 & 0.893 & 0.844 & 0735 & 0.577 \\
\hline & 0.3 & 0.937 & 0.928 & 0.912 & 0.883 & 0.824 & 0.708 & 0.572 \\
\hline \multirow{2}{*}{$t_{2}=0.60 a$} & 0.0 & 0.937 & 0925 & 0.904 & 0.867 & 0.796 & 0.676 & 0.568 \\
\hline & -0.3 & 0.935 & 0.920 & 0.893 & 0.842 & 0.755 & 0.643 & 0.564 \\
\hline \multirow{2}{*}{$t_{3}=0.90 \mathrm{a}$} & -0.6 & 0.934 & 0.914 & 0.873 & 0.800 & 0.701 & 0.614 & 0.561 \\
\hline & -0.9 & 0.952 & 0.975 & 0.877 & 0.746 & 0.652 & 0.595 & 0.559 \\
\hline
\end{tabular}

\begin{tabular}{|c|c|c|c|c|c|c|c|c|}
\hline \multirow{3}{*}{$t_{1}=1.2 a$} & 0.9 & 0.843 & 0.839 & 0.839 & 0.847 & 0.868 & 0.910 & 0.988 \\
\hline & 0.6 & 0.843 & 0.840 & 0.843 & 0.855 & 0.879 & 0.924 & 0995 \\
\hline & 0.3 & 0.843 & 0.842 & 0.849 & 0.856 & 0.895 & 0.939 & 1.002 \\
\hline $2.4 a$ & 0.0 & 0.843 & 0.846 & 0.859 & 0.883 & 0.916 & 0.958 & 1.009 \\
\hline & -0.3 & 0.844 & 0.855 & 0.879 & 0.911 & 0.946 & 0.981 & 1.017 \\
\hline $3.6 \mathrm{a}$ & -0.6 & 0.847 & 0.881 & 0.322 & 0.960 & 0.990 & 1.011 & 1.024 \\
\hline & -0.9 & 0.883 & 0.994 & 1.041 & 1.057 & 1.056 & 1.048 & 1.03 \\
\hline
\end{tabular}

\begin{tabular}{|c|c|c|c|c|c|c|c|c|}
\hline \multirow{3}{*}{$t_{1}=5 a$} & 0.9 & 0.925 & 0.943 & 0.963 & 0.985 & 1.010 & 1.038 & 1.070 \\
\hline & 0.6 & 0.926 & 0.946 & 0.967 & 0.991 & 1.016 & 1.042 & 1.071 \\
\hline & 0.3 & 0.927 & 0.949 & 0.973 & 0.997 & 1.022 & 1.047 & 1.073 \\
\hline \multirow{2}{*}{$t_{2}=10 a$} & 0.0 & 0.928 & 0.955 & 0.980 & 1.005 & 1.029 & 1.052 & 1.074 \\
\hline & -0.3 & 0.931 & 0.962 & 0.990 & 1.014 & 1.037 & 1.057 & 1.075 \\
\hline \multirow{2}{*}{$t_{3}=15 a$} & -0.6 & 0.935 & 0.974 & 1.003 & 1.027 & 1.046 & 1.063 & 1.077 \\
\hline & -0.9 & 0.948 & 0.996 & 1.024 & 1.043 & 1.057 & 1.069 & 1.078 \\
\hline
\end{tabular}

* Three uniform layers on an insulating substrate. 
In Exercise I, above, the method of images was used to find correction factors for a single isolated layer by admixing two possible current distributions on the contacts. In Exercise II, equivalent single isolated layer factors were found using the SG approach [by setting $\beta_{1}=1$ and $B_{2}=0$. in eq $\left.(A 12)\right]$. Both of these methods require lengthy calcu= lations. For the purpose of making rapid calculations of correction factors using parallel superposition, an empirical expression for $H(t)$ has been developed. The expression used is hyperbolic, having asymptotes for small and large slab thicknesses which coincide with the exact form of $H(t)$ calculated by the admixture of currents and subsequent application of the method of images as done in Exercise I above. The hyperbolic expression, having an easily calculated second derivative for use in the parallel superposition method, is:

$$
H(t)=\left[\left(1-\frac{2 a}{\pi s}\right)^{2}+\left(\frac{2 a \ln (s / a)}{\pi t}\right)^{2}\right]^{-\frac{1}{2}} .
$$

Numerical values given by this empirical expression are listed in table A8 for a single isolated slab with various values of $t$, using $s=40 a$. The same table lists values, for these same cases calculated using admixed current distributions under the contacts, i.e., the exact results from A.2 of this appendix and, additionally, values for $\mathrm{H}$ calculated using the SG method. Ratios of empirical to exact values, and of SG to exact value are also given. It is here seen that the results from the SG assumption regarding current distribution under the contact are not significantly different from those with the more general current distribution used in section A.2 to better satisfy the requirement of equipotential under the contact. The values listed for admixed current distributions (the first column of table A8) were used to plot figure 1 of this report. 
Table A8 - Reciprocal Correction Factors for a Single Isolated Layer of Thickness $t^{*}$.

\begin{tabular}{|c|c|c|c|c|c|}
\hline \multirow[b]{2}{*}{$\log _{10}(t / a)$} & \multirow[b]{2}{*}{ exact } & \multirow[b]{2}{*}{ SG } & \multirow[b]{2}{*}{ empir } & \multicolumn{2}{|c|}{ Ratios } \\
\hline & & & & $\frac{\mathrm{SG}}{\text { exact }}$ & empir \\
\hline-1.0 & 0.0426 & 0.0407 & 0.0425 & 0.9569 & 0.9996 \\
\hline-0.8 & $\begin{array}{ll}0 & 0673\end{array}$ & 0.0644 & 0.0673 & 0.9572 & 1.0007 \\
\hline-0.6 & 0.1058 & 0.1016 & 0.1064 & 0.9598 & 1.0052 \\
\hline-0.4 & 0.1645 & 0.1590 & 0.1672 & 0.9669 & 1.0168 \\
\hline-0.2 & 0.2503 & 0.2450 & 0.2597 & 0.9788 & 1.0377 \\
\hline 0.0 & 0.3689 & 0.3656 & 0.3927 & 0.9909 & 1.0645 \\
\hline 02 & 05166 & 0.5162 & 0.5622 & 0.9993 & 1.0883 \\
\hline 0.4 & 0.6726 & 0.6753 & 0.7367 & 1.0039 & 1.0953 \\
\hline 0.6 & 08084 & 0.8134 & 0.8716 & 1.0062 & 1.0782 \\
\hline 0.8 & 0.9058 & 09125 & 0.9505 & 1.0074 & 1.0493 \\
\hline 1.0 & 0.9647 & 0.9723 & 0.9884 & 1.0079 & 1.0246 \\
\hline
\end{tabular}

* Factors are calculated: exact - from admixed current distributions using eqs (A3) and (A6), SG - from eq (A11), and empir - from eq (A18). 
Universal Data Reduction Program

A general program utilizing both the parallel superposition and local slope techniques for interpreting spreading resistance data is listed at the end of this appendix. Also provided is the list of variables for the program. As listed, the program is written for the Hewlett-Packard $9830 \mathrm{~A}$ programmable calculator. The program contains three semiindependent subprograms: a data logging routine, a data reduction routine, and plotting and graph scale subroutines for deriving the associated printer/plotter. The function of the program is to log raw spreading resistance values as they appear at the digital output of the spreading resistance probe; correct them for sampling volume if desired; convert them to either carrier concentration, resistivity, or conductivity; and plot them on a profile graph. (The plotting and graph scale subroutines are not included in the listing because they are specific to the graph format and plotter used, but are available on request. *) The program requires interaction from the operator; when it is run, the calculator display shows a prompt, or message, whenever an input is required from the keyboard.

The normal flow of the program begins with the loading of carrier concentrations and resistivities for 32 standard calibration specimens (through line 50). Lines 60-220 read five standard resistances and up to 140 data points from the digital output of the spreading resistance probe. If fewer than 140 points are run, the data logging loop must be interrupted by pressing the STOP key, and the program must be continued at line 230. Lines 230-250 determine and execute a branch to a number of different places in the program.

The data reduction section begins at line 260. Prestored spreading resistance values appropriate to the probe loading and specimen surface orientation are loaded through line 300. Lines 310-350 define the contact radii to be used in the following calculations. The values given are those used for data reductions contained in this report. They were obtained from the probe spacing experiment on diffused specimens. New values as appropriate would be inserted by the user. Lines 380-430 input experimental parameters, including bevel angle and location information. A loop begins at line 440 which is cycled for each layer of different conductivity type in the profile. Conductivity type, correction scheme, and last (shallowest) point for the layer in question are specified through line 510, and three constants needed later are precalculated at lines 520-540. At line 550 another loop begins which is cycled for each point in the layer. The uncorrected spreading resistance is calculated from raw data at line 560, and line 570 temporarily sets the sampling volume correction factor to unity (in the event that no correction has been specified). The local slope of the profile is determined at line

* Available from D. H. Dickey, Solecon Laboratories, 3001 Red Hill Avenue, Bldg. 3, Suite 106, Costa Mesa, California 92626. 
590, and the desired correction subroutine is called out at line 600. The spreading resistance is corrected for sampling volume at line 620, and the calibration curve look-up subroutine is called at line 640. This subroutine returns interpolated values of resistivity, carrier concentration, and conductivity based on the corrected spreading resistance of the point in question. The depth for the point is calculated at line 660, and the plot subroutine is called out at line 670. After data for each point specified in the layer is reduced and plotted, a test is made at line 720 to determine whether the bevel edge has been reached. If not, the program branches back to line 440 to input the conductivity type of the next layer. When the bevel edge is reached, the graph scales are printed (line 750), and the program branches back to line 230. From line 230, the operator can branch to the data logging routine or can restart the data reduction from the same data after changing some of the parameters. When line 230 is accessed after logging new data, the operator can branch directly to line 360 in the event that the same probe load and orientation are appropriate.

In the local slope subroutine, lines 800-860, the substrate point number is first calculated at line 820. It is the shallower of the deepest point in the layer or a point $\mathrm{N} 3$ increments deeper than the point in question. The uncorrected spreading resistance for the substrate point is calculated from raw data at line 830, and the substrate factor is calculated [according to eq (24)] at line 840. The value of the sampling volume correction factor is calculated [according to eq (22), using $\mathrm{K}_{3}$ for $\mathrm{K}_{1} \mathrm{~K}_{2}$ ] at line 850 and is returned to the main program.

In the parallel superposition subroutine, lines 880-940, the second term of eq (16) is first calculated. The second derivative of $H(t)$, from eq (A15), is multiplied by the conductivity of each point and summed through line 920. The sum $\mathrm{sl}$ is multiplied by its coefficient to complete the second term of eq (16), and this is added to the slope term to give the sampling volume correction factor at line 930.

For routine use, the local slope scheme is used almost exclusively except when no correction is desired. The parallel superposition scheme is only used as a check on simple diffusions, where surface resistivity is lower than subsurface or substrate resistivity. It cannot be used in cases where subsurface resistivity is lower than that at the surface, whereas the local slope scheme handles these cases easily. The accuracy of the local slope scheme is not particularly sensitive to the choice of substrate factor except in a few special cases, but an improved formula for it is probably desirable. The substrate point has been specified N3 points deeper on the profile because the second derivative of $H(t)$ peaks at this depth. In effect, the substrate factor approximates the summation in eq (16) with a single point. 
$10 \operatorname{DIM}$ AS $[2,2], N S[2,16], \operatorname{PS}[2,16], \operatorname{RS}[2,16], \operatorname{SS}[140]$, UI [5] , VI [140] , YS [3]

20 LOAD DATA $21, \mathrm{P}$

30 REM P ARRAY CONTAINS LOGS OF CALIBRATION SAMPLE RESISTIVITIES

40 LOAD DATA $22, \mathrm{~N}$

50 REM N ARRAY CONTAINS LOGS OF CALIBRATION SAMPLE CONCENTRATIONS

60 FOR I=1 TO 5

70 REM READING PROBE OUTPUT FOR 5 STANDARD RESISTANCES

80 DISP "PRINT FOR R=10" 2 * I-2

90 WAIT 1000

100 ENTER $(1, *) A, V$

$110 U[I]=V^{*} 1 E+04$

120 DISP I,U[I]

130 WAIT 1000

140 NEXT I

150 FOR I=1 TO 140

160 REM READING PROBE OUTPUT FOR DATA POINTS

170 REM INTERRUPT LOOP AND CONTINUE AT LINE 230 IF LESS THAN 140 POINTS RUN 180 ENTER $\left(1,{ }^{\star}\right) A, V$

$190 \mathrm{~V}[\mathrm{I}]=\mathrm{V} * 1 \mathrm{E}+04$

200 DISP I,V[I]

210 WAIT 1000

220 NEXT I

230 DISP "WHAT NEXT";

240 INPUT D

250 GOTO D OF $60,260,360,440,750,780$

260 DISP "PROBE LOAD, ORIENTATION";

270 INPUT L1,Q

$280 \mathrm{~L}=\mathrm{INT}(\mathrm{L} 1 / 10)$

290 LOAD DATA $\left(\mathrm{L}+2{ }^{*} \mathrm{Q}+22\right), \mathrm{R}$

300 REM R ARRAY CONTAINS IOGS OF CALIBRATION SAMPLE RESISTANCES

$310 \mathrm{~A}[1,1]=1.1$

$320 \mathrm{~A}[1,2]=2.1$

$330 \mathrm{~A}[2,1]=0.5$

$340 A[2,2]=1.1$

350. REM A $(T, L)$ IS CONTACT RADIUS FOR TYPE(T) AND LOAD(L)

$360 \mathrm{Fl}=1$

370 REM F1 IS A FLAG USED IN PLOT SUBROUTINE

380 DISP "PROBE SPACING";

390 INPUT $S$

400 DISP "BEVEL ANGLE, X-STEP";

410 INPUT $A, B$

420 DISP "BEVEL EDGE, START PT";

430 INPUT N0,N2

440 DISP "TYPE(P OR N)";

450 INPUT BS

$460 \mathrm{~T}=\mathrm{POS}(\mathrm{BS}$, "P") +2 *POS (BS, "N")

470 IF $T=0$ THEN 230

480 DISP "CORRECTION";

490 INPUT $C$

500 DISP "STOP POINT";

510 INPUT N1

$520 \mathrm{~K} 1=2{ }^{\star} \mathrm{A}[\mathrm{T}, \mathrm{L}]{ }^{\star} \mathrm{LOG}(\mathrm{S} / \mathrm{A}[\mathrm{T}, \mathrm{L}]) / \mathrm{PI}$

$530 \mathrm{~K}=\mathrm{K} 1 / \mathrm{A} / \mathrm{B}$

$540 \mathrm{~K} 4=(1-2 \star \mathrm{A}[\mathrm{T}, \mathrm{L}] / \mathrm{PI} / \mathrm{S})-2$

550 FOR N=N2 TO N1 STEP -1

$560 \mathrm{R} 0=4+4 *(\mathrm{~V}[\mathrm{~N}]-\mathrm{U}[3]) /(\mathrm{U}[4]-\mathrm{U}[2])$ 
$570 \mathrm{~F}=1$

580 IF $C=0$ THEN 620

$590 \mathrm{M}=4.6 / \mathrm{A} / \mathrm{B} *(1+(\mathrm{N}=\mathrm{N} 1)+(\mathrm{N}=\mathrm{N} 2)) *(\mathrm{~V}[\mathrm{~N}-1+(\mathrm{N}=\mathrm{N} 1)]-\mathrm{V}[\mathrm{N}+1-(\mathrm{N}=\mathrm{N} 2)]) /(\mathrm{U}[4]-\mathrm{U}[2])$

600 GOSUB C OF 800,880

610 REM BRANCHING TO EITHER IOCAL SLOPE OR PARALLEL SUPERPOSITION SUBROUTINE

$620 \mathrm{R}=\mathrm{R} 0-\mathrm{LGTF}$

630 REM RO HAS NOW BEEN CORRECTED FOR SAMPLING VOLUME

640 GOSUB 960

650 REM BRANCHING TO CALIBRATION CURVE LOOK-UP SUBROUTINE

$660 \mathrm{X}=(\mathrm{N}-\mathrm{NO}){ }^{*} \mathrm{~A}{ }^{*} \mathrm{~B}$

670 GOSUB 1120

680 REM BRANCHING TO PLOT SUBROUTINE

690 NEXT N

$700 \mathrm{~N} 2=\mathrm{N} 1-1$

710 REM SET NEW START POINT TO (OLD STOP POINT - 1)

720 IF N2 <= NO THEN 750

730 GOTO 440

740 REM RETURN TO REDUCE NEXT LAYER

750 GOSUB 1620

760 REM BRANCHING TO SCALE GRAPH

770 GOTO 230

780 END

790 REM

800 REM

810 N3=INT $(K)$

$820 \mathrm{~N} 4=(\mathrm{N}+\mathrm{N} 3) *((\mathrm{~N}+\mathrm{N} 3)<=\mathrm{N} 2)+\mathrm{N} 2 *((\mathrm{~N}+\mathrm{N} 3)>\mathrm{N} 2)$

$830 \mathrm{R} 1=4+4 *(\mathrm{~V}[\mathrm{~N} 4]-\mathrm{U}[3]) /(\mathrm{U}[4]-\mathrm{U}[2])$

$840 \mathrm{~K} 3=1+(8 * \mathrm{LOG}(\mathrm{S} / \mathrm{A}[\mathrm{T}, \mathrm{L}]) / \mathrm{PI} / \mathrm{PI}=1) * 2 / \mathrm{PI}$ *ATN $(\mathrm{R} 0-\mathrm{R} 1)$

$850 \mathrm{~F}=-\mathrm{K} 1 * \mathrm{M} / 2+\mathrm{SQR}(\mathrm{K} 1 * \mathrm{M} * \mathrm{~K} 1 * \mathrm{M} / 4+\mathrm{K} 3)$

860 RETURN

870 REM

880 REM

$890 \mathrm{~S} 1=0$

900 FOR $\mathrm{I}=1$ TO N2-N

$910 \mathrm{~S} 1=\mathrm{S} 1+3 * I * K * K * \mathrm{~K} 4 * \mathrm{~S}[\mathrm{~N}+\mathrm{I}] /\left(\mathrm{K} 4{ }^{*} I * I+K{ }^{*} \mathrm{~K}\right)-2.5$

920 NEXT I

$930 \mathrm{~F}=-\mathrm{K} 1 * \mathrm{M}+0.0002 * \mathrm{~A}[\mathrm{~T}, \mathrm{~L}] * 10^{\wedge} \mathrm{R} 0 \mathrm{~K}^{*} \mathrm{~S} 1$

940 RETURN

950 REM1

960 REM

$970 \mathrm{~J}=16$

980 IF $R>R[T, J]$ THEN 1020

990 IF $J=1$ THEN 1020

$1000 \mathrm{~J}=\mathrm{J}-1$

1010 GOTO 980

1020 IF $\mathrm{J}=16$ THEN 1060

$1030 Y[1]=(R-R[T, J]) *(N[T, J+1]-N[T, J]) /(R[T, J+1]-R[T, J])+N[T, J]$

$1040 Y[2]=(R-R[T, J]) *(P[T, J+1]-P[T, J]) /(R[T, J+1]-R[T, J])+P[T, J]$

1050 GOTO 1080

$1060 Y[1]=R[T, 16]-R+N[T, 16]$

$1070 Y[2]=R-R[T, 16]+P[T, 16]$

$1080 Y[3]=-Y[2]$

$1090 \mathrm{~S}[\mathrm{~N}]=10^{\wedge} \mathrm{Y}[3]$

1100 RETURN

1110 REM

1120 REM

PLOT SUBROUTINE BEGINS 
Units

I. Dimensioned Variables - Integer Precision

U(5) - Digital voltmeter reading at output of probe, for five standard resistances $10^{-4} \mathrm{~V}$

V(140) - Digital voltmeter reading at output of probe, for data points

II. Dimensioned Variables - Split Precision
$A(2,2)$ - Contact radius, for two conductivity types and two probe loadings
$\mu \mathrm{m}$
$\mathrm{N}(2,16)$ - Logarithm of carrier concentration for calibration sample, for 16 specimens of each conductivity type
$P(2,16)$ - Logarithm of resistivity for calibration sample, for 16 specimens of each conduc- tivity type
$R(2,16)$ - Logarithm of spreading resistance for calibration sample, for 16 specimens of each conductivity type
$S(140)$ - Conductivity for each data point
$\mathrm{Y}(3)$ - Logarithm of (1) carrier concentration, (2) resistivity, and (3) conductivity, for data point

III. Simple Variables
A
- Bevel angle
rads
B
- X-step
$\mu \mathrm{m}$
F
- Sampling volume correction factor
K
- Defined constant (see program line 530)
$\mathrm{Kl}$
- Defined constant (see eq (20))
$\mu \mathrm{m}$
K3
- Substrate factor (see eq (24))
K4
- Defined constant
L1
- Probe loading
9
M
- Profile slope
(decades) $/ \mu \mathrm{m}$
NO
- Bevel edge point number
N1
- Stop point number
N2
- start point number 
III. Simple Variables (continued)

Units

N3 - The number of points to substrate point

N4 - Point number for substrate point

$\mathrm{R}$ - Logarithm of corrected spreading resistance

RO

- Logarithm of uncorrected spreading resistance

Rl - Logarithm spreading resistance for substrate point

$S$

- Probe spacing

Sl

- Sum of conductivity $\times \Delta^{2} \mathrm{H}$ (see eq (16))

$(\Omega \cdot \mathrm{cm})^{-1}$

IV. Flags and Pointers

C

- Correction scheme pointer

0 - no correction

1 - local slope

2 - parallel superposition

D

- Branching pointer, range 1-6

Fl - Flag used to set up plot scales

T - Conductivity type flag

1 - p-type

2 - n-type

L - Probe load flag

1 - 10-g load

2 - 20-g load

Q

- Orientation flag

0 - (100) orientation

1 - (111) orientation 


\section{APPENDIX C \\ CORRECTION FACTOR FOR UNIFORM-RESISTIVITY WEDGE}

The effect of bevel angle on spreading resistance measurements has been explored by solving the potential distribution problem represented by two disk contacts on one surface of an infinite homogeneous wedge. If the wedge angle is taken as an odd-integral submultiple of $\pi$, the problem can be solved using the method of images with a finite array of images. The solution is approximated by taking the images as point sources rather than disk-like, although it is straightforward (though laborious) to write the exact solution in terms of Legendre polynomials. The model assumed is shown in figure $\mathrm{Cl}$, where the two contacts are aligned parallel to the axis of the wedge at a place where the local thickness is $t$. The correction factor in the point-image approximation is

$$
F=\frac{2 a}{(2 N+1) t} \sum_{M=0}^{N-1}\left\{\frac{1}{\cos \left(\frac{\pi}{2} \cdot \frac{2 M+1}{2 N+1}\right)}-\frac{1}{\left[\cos ^{2}\left(\frac{\pi}{2} \cdot \frac{2 M+l}{2 N+1}\right)+\lambda^{2}\right] 1 / 2}\right\}
$$

where $\lambda$ is given by

$$
\lambda=\frac{\pi s}{2(2 N+1) t}
$$

and $\mathrm{N}$ is the number of images, related to the wedge angle according to

$$
\alpha=\frac{\pi}{2 \mathrm{~N}+1}
$$

Correction factors were computed from eq (Cl) for a variety of wedge angles, for two values of local thickness, and two values of probe spacing. The results are shown in figures $\mathrm{C} 2$ and $\mathrm{C} 3$.

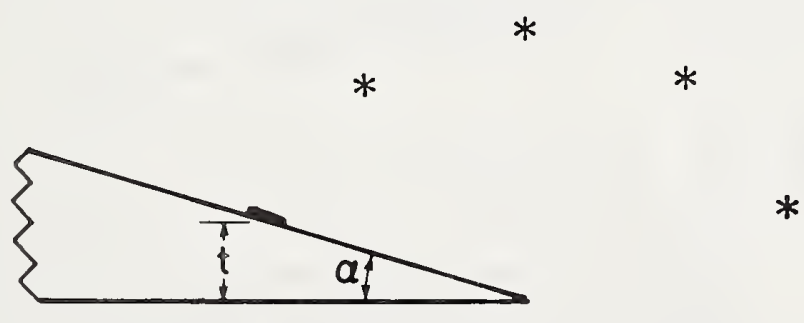

* $*$

* $*$

Figure Cl. Image array for infinite homogeneous wedge. 


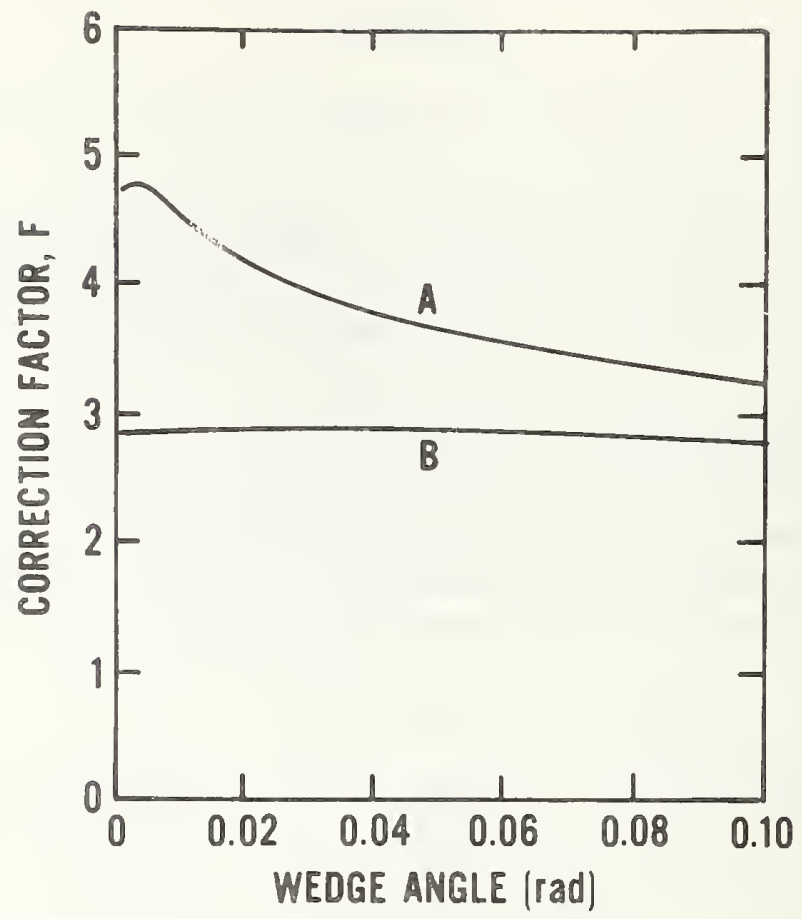

Figure C2. Sampling volume correction factor for uniform wedge with local thickness equal to contact radius. Curve $A$, probe spacing equal to 400 contact radii; curve $B$, probe spacing equal to 40 contact radii.

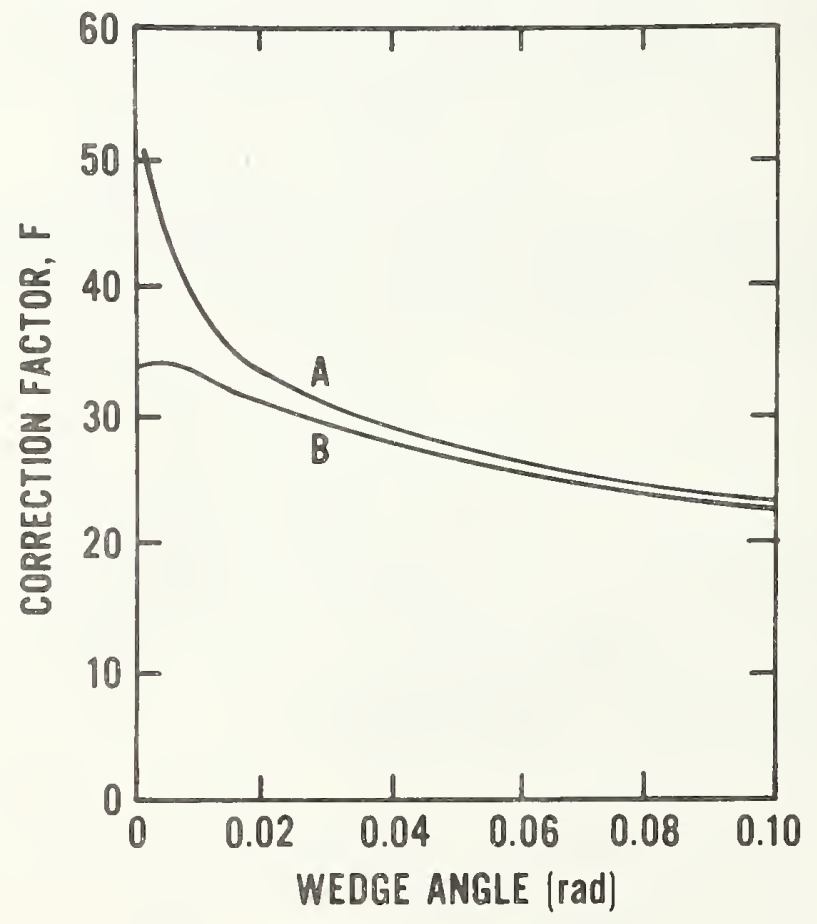

Figure C3. Sampling volume correction factor for uniform wedge with local thickness equal to 0.1 contact radii. Curve $A$, probe spacing equal to 400 contact radii; curve B, probe spacing equal to 40 contact radii. 


\section{APPENDIX D}

\section{SPECIAL OPERATIONAL PROBLEMS OF THE LOCAL SLOPE METHOD AND SUGGESTED ALTERNATIVES}

A problem was encountered when using the local slope method for analyzing seemingly anomalous data which occurred near the bevel edge of isolated layers. The problem was the calculation of nonphysical (negative or imaginary) values for $F$. It is shown below that the anomalous data are not the prime cause of the unacceptable values for $F$, but they do complicate analysis of the problem. The existence of such anomalous data, which are more obvious on thin isolated layers, is believed due to the asymmetry in the conduction path between the probes when in proximity to the bevel edge. This same asymmetry in potential distribution near the bevel edge should cause similar, if less recognizable, data anomalies in other layer structures and will affect the accuracy of analysis for any correction factor algorithm chosen [5].

Whatever the cause of such anomalous data, they are typically seen on measurements of a thin isolated layer as nonmonotonic or turnaround behavior for data near the bevel edge. Abnormally high spreading resistance values are seen at the bevel edge, followed by decrease to some minimum value, then rapidly increasing values of spreading resistance following the normal behavior for thin isolated layers as shown in figure Dl. This data turnaround is generally found to increase with increasing s/a values in a manner similar to that shown in figure 18 for the uniform top surface of a layer. The result of the nonmonotonic data is that local slope values, calculated for coordinates beginning at the bevel edge, are negative for a few points, then increase through zero to normal positive values.

It is noted that for thin isolated layers such nonmonotonic data are contradictory to the assumptions of the parallel superposition model upon which the isolated layer limit of the local slope model is founded. According to the parallel superposition model, as supported by the results of the probe spacing experiment in section 3.1, the spreading resistance measured at any point on a sectioned isolated layer is proportional to the sheet resistance of the layer beneath the point of measurement.

Since the concept of parallel superposition is supported by the experimental results of section 3.1 , it was necessary to question whether the anomalous data or the local slope extension of paral.lel superposition was responsible for the nonphysical values for F. Tables DI to D5 were constructed for various values of local slope. Equation (22a) using the function $\mathrm{K}_{3}$ was used to calculate $\mathrm{F}$ for various values of $\mathrm{s} / \mathrm{a}$ and of $\mathrm{R}_{\mathrm{o}} / \mathrm{R}$. For the same values of local slope and of $\mathrm{s} / \mathrm{a}, \mathrm{F}$ was also calculated using $\mathrm{K}_{1} \mathrm{~K}_{2}$ or unity in place of $\mathrm{K}_{3}$.

Two features found in the tables indicate that anomalous data are not the primary cause of nonphysical $F$ values. The first is that use of $\mathrm{K}_{1} \mathrm{~K}_{2}$ or unity always results in positive $\mathrm{F}$ values regardless of local 


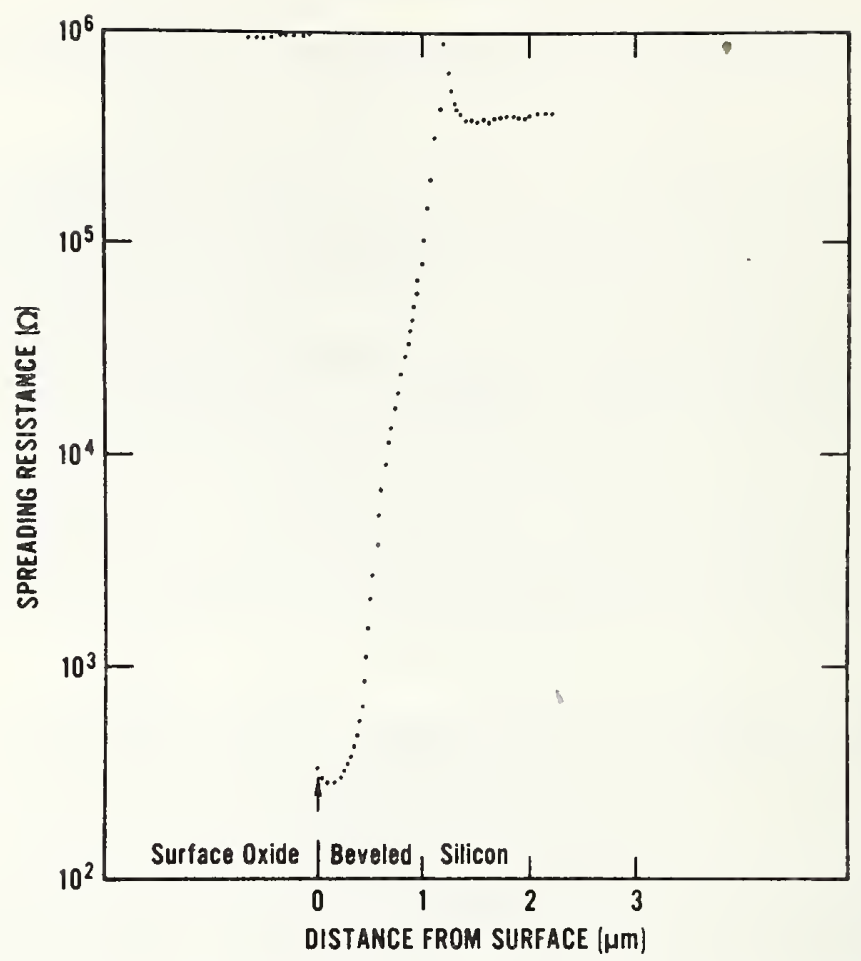

Figure Dl. Spreading resistance data for junction-isolated implanted specimen illustrating the type of local slope behavior near the top surface which gives nonphysical values for the correction factor when analyzed by eq $(22 a)$ or $(22 b)$.

slope value. The second is that use of $K_{3}$ results in some nonphysical $F$ values even for small positive local slope values.

The only exception to the general pattern of nonphysical $F$ values, when using the form $K_{3}$ with small $R_{0} / R_{s}$ in eq (22a), occurs for very small values of $\mathrm{s} / \mathrm{a}$. This result, only seen near the limit of physicalIy attainable s/a values, is regarded as an accident. It is noted that the values for $F$ under such circumstances, although positive, are noticeably different from values calculated using the forms $\mathrm{K}_{1} \mathrm{~K}_{2}$ or unity.

The fact that nonphysical values can be calculated for $F$ for certain combinations of local slope and measurement condition does not invalidate the derivations or algorithm construction embodied in this work. For many layers of this type, the strict formalism of parallel superposition, eq (16), could always be used as outlined in Appendix B; no such spurious results are encountered for this formalism. However, it is assumed that the local slope formalism is more appropriate for multilayer structures, even if slight modifications must be made for application to isolated layers. It must be remembered that the term $\mathrm{K}_{3}$ was originally hypothesized as a replacement for $\mathrm{K}_{1} \mathrm{~K}_{2}$ in eq (22) to make the equation for $\mathrm{F}$ more generally 
Tables D - Correction Factor $F$ Based on Local Slope Using $K_{3}$ for Various Values of $R_{0} / R_{5}$ and Also Using $K_{7} K_{2}$ and Unity to Replace $K_{3}$ : Case of $a=2 \mu \mathrm{m}$.

Táble D1: Case of Local Slope, $m=-0.5$

\begin{tabular}{|c|c|c|c|c|c|c|c|c|}
\hline & 1 & 0.5 & 0.1 & 0.01 & 0.001 & 0.0001 & $\mathrm{~K}_{1} \mathrm{~K}_{2}$ & 1 \\
\hline 10 & .51 & .44 & .32 & .23 & .19 & .74 & .82 & .51 \\
\hline 20 & .43 & .32 & .14 & 0 & -.08 & -.17 & .87 & .43 \\
\hline 40 & .37 & .23 & 0 & -.18 & -.29 & -.41 & .92 & .37 \\
\hline 60 & .34 & .19 & -.06 & -.26 & -.39 & -.52 & .94 & .34 \\
\hline 80 & .32 & .17 & -.10 & -.32 & -.44 & -.59 & .95 & .32 \\
\hline
\end{tabular}

Table D2: Case of Local Slope, $m=-0.1$

\begin{tabular}{r|rrrrrr|rr|r}
$s / a$ & 1 & 0.5 & 0.1 & 0.01 & 0.001 & 0.0001 & $k_{1} K_{2}$ & 1 \\
\hline 10 & .86 & .77 & .62 & .49 & .42 & .34 & 1.23 & .86 \\
20 & .83 & .68 & .38 & 0 & $\mathbf{i}$ & $\mathbf{i}$ & 1.38 & .83 \\
40 & .79 & .57 & .01 & $\mathbf{i}$ & $\mathbf{i}$ & $\mathbf{i}$ & 1.51 & .79 \\
60 & .77 & .52 & $\mathbf{i}$ & $\mathbf{i}$ & $\mathbf{i}$ & $\mathbf{i}$ & 1.58 & .77 \\
80 & .76 & .47 & $\mathbf{i}$ & $\mathbf{j}$ & $\mathbf{i}$ & $\mathbf{i}$ & 1.62 & $.7 \epsilon$
\end{tabular}


Table D3: Case of Local Slope, $m=0$

\begin{tabular}{|c|c|c|c|c|c|c|c|c|}
\hline$s / a$ & 1 & 0.5 & 0.1 & 0.01 & 0.001 & 0.0001 & $k_{1} k_{2}$ & 1 \\
\hline 10 & 1 & .91 & .75 & .62 & .56 & .47 & 1.35 & 1 \\
\hline 20 & 1 & .34 & .54 & 0 & $i$ & $i$ & 1.55 & 1 \\
\hline 40 & 1 & .78 & 0 & $i$ & $i$ & i & 1.73 & 1 \\
\hline 60 & 1 & .73 & $i$ & i & $i$ & $i$ & 1.82 & 1 \\
\hline 80 & 1 & .70 & $i$ & i & $i$ & $i$ & 1.86 & 1 \\
\hline
\end{tabular}

Table 04: Case of Local Slope, $m=0.1$

\begin{tabular}{|c|c|c|c|c|c|c|c|c|}
\hline$s / a$ & 1 & 0.5 & 0.1 & 0.01 & 0.001 & 0.0001 & $k_{1} K_{2}$ & 1 \\
\hline 10 & 1.16 & 1.07 & .92 & .79 & .72 & .64 & 1.53 & $i .16$ \\
\hline 20 & 1.21 & 1.06 & .76 & .38 & $i$ & $i$ & 1.76 & 1.21 \\
\hline 40 & 1.27 & 1.05 & .49 & $i$ & $i$ & $i$ & 1.99 & 1.27 \\
\hline 60 & 1.29 & 1.04 & i & $i$ & $i$ & $i$ & 2.10 & 1.29 \\
\hline 80 & 1.32 & 1.03 & $i$ & $i$ & $i$ & i & 2.18 & 1.32 \\
\hline
\end{tabular}


Table 05: Case of l.ocal Slope, $\mathrm{n}=0.5$

\begin{tabular}{l|cccccc|c|c|c}
\hline & 1 & 0.5 & 0.1 & 0.01 & 0.001 & 0.0001 & $K_{7} K_{2}$ & 1 \\
\hline 10 & 1.98 & 1.90 & 1.79 & 1.70 & 1.66 & 1.61 & 2.23 & 1.97 \\
20 & 2.34 & 2.24 & 2.05 & 1.91 & 1.83 & 1.74 & 2.72 & 2.33 \\
40 & 2.72 & 2.57 & 2.35 & 2.17 & 2.06 & 1.94 & 3.25 & 2.71 \\
60 & 2.95 & 2.79 & 2.55 & 2.35 & 2.22 & 2.09 & 3.54 & 2.98 \\
80 & 3.11 & 2.97 & 2.69 & 2.47 & 2.35 & 2.20 & 3.74 & 3.12
\end{tabular}

applicable. In particular, it was intended to make the local slope formalism applicable to a wider variety of layer and substrate resistivities and thicknesses than were embodied in the two limiting forms used as starting points. The term in $\mathrm{R} / \mathrm{R}$ had the effect of smoothing the transition between the limiting (large nếgative slope and large positive slope) forms for $\mathrm{F}$. In addition, for the case of a general isolated layer, the term in $R_{0} / R_{s}$ was intended to incorporate the contribution due to sublayers lying deeper than one contact radius as was done by the second term in the pure parallel superposition formalism [eq (16)] .

Accounting for such deep sublayer effects is clearly not necessary for thin, $t<a$, isolated layers, and it makes only a small difference in the analysis of moderately thicker layers. Hence, the added flexibility built into $\mathrm{K}_{3}$ is clearly expendable for analysis of many well-defined isolated layers and the use of a form other than $k_{3}$ in eq (22) is advisable.

For moderate to large positive values of local slope, little difference results from use of $\mathrm{K}_{3}, \mathrm{~K}_{1} \mathrm{~K}_{2}$, or unity as the second term under the radical sign in the equation for ${ }^{2} \mathrm{~F}$. A meaningful difference among these choices only occurs for small values of local slope, i.e., near the bevel edge of an isolated layer. Since no analytical model is available for bevel-edge effects, the correct manner of analyzing data taken near a specimen bevel edge is not established. Any procedure used to calculate the sampling volume factor, $F$, will be an approximation. This limitation also occurs for all other methods of calculating the sampling volume factor which are based on models with lateral symmetry, including the method of SG.

Some manner of calculating $\mathrm{F}$, other than that using the term $\mathrm{K}_{3}$ must still be chosen. The choices mentioned, $\mathrm{K}_{1} \mathrm{~K}_{2}$ and unity, are not the only 
possible replacements, but they are seen to be reasonable choices. As mentioned, for moderate to large local slope values, little difference results from any of the choices. For small values of local slope, use of either $\mathrm{K}_{1} \mathrm{~K}_{2}$ or unity gives values for $\mathrm{F}$ near unity. Such a value is appropriate since, when the true correction is uncertain, the least correction possible, i.e., unity, is the safest. Further, the tendency of the data to show a near zero value of local slope near the surface indicates that the effect of the underlying junction is no longer dominant in the conduction between the probes. Hence, a correction near unity is not unreasonable. It is noted from the tables that attempts to retain $\mathrm{K}_{3}$ in the equation for $F$ and to modify the data set instead, as by replacing negative values of local slope by zero or by extrapolating the minimum measured resistance to the surface, simply result in replacing a negative $F$, for $m<0$, by an imaginary $F$, for $m=0$. When correct nearsurface data analysis is particularly important, replacement of $\mathrm{K}_{3}$ also appears preferable for somewhat thicker isolated layers despite the possible need to account for the deeper layer contributions that are built into $\mathrm{K}_{3}$. Such a case exists when the sheet resistance of moderate depth diffusions needs to be evaluated from the integration of the resistivity profile, where near-surface data make the dominant contribution to the sheet resistance calibration. Then replacement of $\mathrm{K}_{3}$ results in relative differences ranging from a fraction of a percent to about 15 percent for data on the steeper part of the profile where $\mathrm{m} \geq 1$. The effect of these changes on the contribution to the layer sheet resistance will generally be small compared to the contribution from reasonably determined values of near-surface points, particularly if $\mathrm{m} \leq 0$ is encountered.

Since the terms $\mathrm{K}_{1} \mathrm{~K}_{2}$ and unity come from hybrid forms more general than the simple isolated layer model, neither is strictly correct. $\mathrm{K}_{1} \mathrm{~K}_{2}$
gives somewhat larger values for $\mathrm{F}$ than does unity; the difference in creases with increasing $\mathrm{s} / \mathrm{a}$ values. The volume correction factor for an isolated layer is normally greater than unity to account for an increase in measured resistance resulting from a foreshortened conducting volume. Near the bevel edge, the measured resistance again increases, apparently due to a missing portion of the specimen, as seen in figure 18, and a correction factor greater than unity can again be argued. It is perhaps judicious, then, to choose the form $\mathrm{K}_{1} \mathrm{~K}_{2}$ as a replacement for $\mathrm{K}_{3}$, since it seems to give a scale of volume correction factors in reasonable agreement with what is expected from consideration of the physical problem.

One possible exception to the recommendation for replacing $\mathrm{K}_{3}$ occurs in the analysis of isolated layers of thyristors and related deep structures. Here the cumulative effect of the loss of deeper layer considerations built into $\mathrm{K}_{3}$ may be more severe than the advantage gained from better interpretation of a few points near the surface. A possible recourse here is simply to retain $\mathrm{K}_{3}$ in the calculation for $\mathrm{F}$ until and unless negative or zero values are calculated for $F$. If zero or negative values are calculated for $F$ for a few points near the surface, the resistivity of those affected points is replaced with the value extrapo- 
lated from points just below the affected region. Again, the strict parallel superposition formalism could be used instead.

The discussion in this appendix assumes ideal, or noise-free, data so that the local slope evaluated from successive pairs of data is a smoothly varying function. In the event of noisy data, it is possible to encounter difficulties of the type described if given pairs of data result in local slope values which run counter to the trend in that part of the profile. Data smoothing is advisable for most effective use of these algorithms if data tend to be noisy. 
U.S. DEPT. OF COMM.

BIBLIOGRAPHIC DATA SHEET

1. PUBLICATION OR REPORT NO.

NBS Spec. Publ. 400-48

4. TITLE AND SUBTITLE

Semiconductor Measurement Technology:

Spreading Resistance Analysis for Silicon Layers with

Nonuniform Resistivity

7. AUTHOR(S)

David H. Dickey and James R. Ehrstein

9. PERFORMING ORGANIZATION NAME AND ADDRESS

NATIONAL BUREAU OF STANDARDS

DEPARTMENT OF COMMERCE

WASHINGTON, DC 20234

Solecon Laboratories

and 3001 Red Hill Avenue

Costa Mesa, CA 92626

12. SPONSORING ORGANIZATION NAME AND COMPLETE ADDRESS (Stroet, City, Stato, $z I P$ )

ARPA

1400 Wilson Boulevard

Arlington, VA 22209

5. Publication Date

May 1979

7. Porberaing Oreanization Code

8. Performing Organ. Report No.

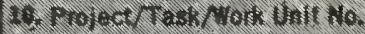

11. Contract/Grant No.

$5-35881$

13. Type gf Report \& Period Covered

$10 / 1 / 75-9 / 30 / 76$

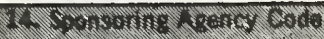

\section{SUPPLEMENTARY NOTES}

This work was funded by the Defense Advanced Research Projects Agency under

ARPA Order 2397 . computer program; SF-185, FIPS Software Summary, is attached.

16. ABSTRACT (A 200-word or los a factual summary of most significant infomation. If documerit includes a significant bibliography or it terature survey, mention it hore.)

A simple mathematical algorithm is developed for the calculation of resistivity depth profiles from spreading resistance measurements on sectioned silicon device structures It is applicable to structures consisting of one or more layers of the same or differing conductivity types. The algorithm accounts for modification of the sampling volum of the spreading resistance probes due to nearby variations in depth of specimen resistivity whether resulting from graded dopant distribution or electrical boundaries either insulating or conducting.

The algorithm is based on limiting case one- and two-dimensional models of the conduction between the probes. Compared with the traditional spreading resistance analysis algorithm based on the three-dimensional boundary value solution of Schumann and Gardn the present work offers greatly enhanced execution times even with a microcomputer, making real time analysis for process control possible.

An experiment to test the accuracy of one of the limiting models used is described. Profiles of a number of diffused layers generated from spreading resistance measurements analyzed by this algorithm are compared with profiles obtained on replicate specimens using another electrical technique. In addition, computer experiments are used for simple layer models to compare results based on this algorithm with results from the Schumann-Gardner approach.

17. KEY WORDS (six to twolve entrios: alphabelical order; capitalize only the firat lotter of the soparated by semicolons)

Dopant profiles; resistivity; resistivity profiles; semiconductor; silicon; spreading resistance.

18. AVAILABILITY

Xnlimited

For Official Distribution. Do Hot Release to NTIS

Order From Sup. of Doc. U.S. Government Printing Office, Washington, DC 20402, SD Stock No. SN $003-003-02061-3$

X] Order From National Technical Information Service (NTIS), Springfield, VA, 22161

\begin{tabular}{|l|c|}
\hline $\begin{array}{l}\text { 19. SECURITY CLASS } \\
\text { (THIS REPORT) } \\
\text { UNCLASSIFIED }\end{array}$ & $\begin{array}{c}\text { 21. NO. OF } \\
\text { PRINTED PAGE } \\
72\end{array}$ \\
\hline $\begin{array}{l}\text { 20. SECURITY CLASS } \\
\text { (THIS PAGE) }\end{array}$ & 22. Price \\
UNCLASSIFIED & \\
\hline
\end{tabular}




\section{NBS TECHNICAL PUBLICATIONS}

\section{PERIODICALS}

JOURNAL OF RESEARCH-The Journal of Research of the National Bureau of Standards reports NBS research and development in those disciplines of the physical and engineering sciences in which the Bureau is active. These include physics, chemistry, engineering, mathematics, and computer sciences. Papers cover a broad range of subjects, with major emphasis on measurement methodology, and the basic technology underlying standardization. Also included from time to time are survey articles on topics closely related to the Bureau's technical and scientific programs. As a special service to subscribers each issue contains complete citations to all recent NBS publications in NBS and nonNBS media. Issued six times a year. Annual subscription: domestic $\$ 17.00$; foreign $\$ 21.25$. Single copy, $\$ 3.00$ domestic; $\$ 3.75$ foreign.

Note: The Journal was formerly published in two sections: Section A "Physics and Chemistry" and Section B "Mathematical Sciences."

\section{DIMENSIONS/NBS}

This monthly magazine is published to inform scientists, engineers, businessmen, industry, teachers, students, and consumers of the latest advances in science and technology, with primary emphasis on the work at NBS. The magazine highlights and reviews such issues as energy research, fire protection, building technology, metric conversion, pollution abatement, health and safety, and consumer product performance. In addition, it reports the results of Bureau programs in measurement standards and techniques, properties of matter and materials, engineering standards and services, nstrumentation, and automatic data processing.

Annual subscription: Domestic, \$1 I.00; Foreign \$13.75

\section{NONPERIODICALS}

- Monographs-Major contributions to the technical literture on various subjects related to the Bureau's scientific nd technical activities.

landbooks-Recommended codes of engineering and indusial practice (including safety codes) developed in cooperaon with interested industries, professional organizations, ind regulatory bodies.

secial Publications-Include proceedings of conferences onsored by NBS, NBS annual reports, and other special ublications appropriate to this grouping such as wall charts, ycket cards, and bibliographies.

pplied Mathematics. Series-Mathematical tables, manU.ls, and studies of special interest to physicists, engineers, lemists, biologists, mathematicians, computer programmers, id others engaged in scientific and technical work.

tional Standard Reference Data Series-Provides quantiinglyive data on the physical and chemical properties of terials, compiled from the world's literature and critically aluated. Developed under a world-wide program codinated by NBS. Program under authority of National ndard Data Act (Public Law 90-396).

\section{BIBLIOGRAPHIC SUBSCRIPTION SERVICES}

following current-awareness and literature-survey bibliaphies are issued periodically by the Bureau:

ogenic Data Center Current Awareness Service. A literaure survey issued biweekly. Annual subscription: Domesc, $\$ 25.00$; Foreign, $\$ 30.00$.

sified Natural Gas. A literature survey issued quarterly. aual subscription: $\$ 20.00$.
NOTE: At present the principal publication outlet for these data is the Journal of Physical and Chemical Reference Data (JPCRD) published quarterly for NBS by the American Chemical Society (ACS) and the American Institute of Physics (AIP). Subscriptions, reprints, and supplements available from ACS, 1155 Sixteenth St. N.W., Wash., D.C. 20056.

Building Science Series-Disseminates technical information developed at the Bureau on building materials, components, systems, and whole structures. The series presents research results, test methods, and performance criteria related to the structural and environmental functions and the durability and safety characteristics of building elements and systems.

Technical Notes-Studies or reports which are complete in themselves but restrictive in their treatment of a subject. Analogous to monographs but not so comprehensive in scope or definitive in treatment of the subject area. Often serve as a vehicle for final reports of work performed at NBS under the sponsorship of other government agencies. Voluntary Product Standards-Developed under procedures published by the Department of Commerce in Part 10, Title 15, of the Code of Federal Regulations. The purpose of the standards is to establish nationally recognized requirements for products, and to provide all concerned interests with a basis for common understanding of the characteristics of the products. NBS administers this program as a supplement to the activities of the private sector standardizing organizations.

Consumer Information Series-Practical information, based on NBS research and experience, covering areas of interest to the consumer. Easily understandable language and illustrations provide useful background knowledge for shopping in today's technological marketplace.

Order above NBS publications from: Superintendent of Documents, Government Printing Office, Washington, D.C. 20402.

Order following NBS publications-NBSIR's and FIPS from the National Technical Information Services, Springfield, Va. 22161.

Federal Information Processing Standards Publications (FIPS PUB)-Publications in this series collectively constitute the Federal Information Processing Standards Register. Register serves as the official source of information in the Federal Government regarding standards issued by NBS pursuant to the Federal Property and Administrative Services Act of 1949 as amended, Public Law 89-306 (79 Stat. 1127), and as implemented by Executive Order 11717 (38 FR 12315, dated May 11, 1973) and Part 6 of Title 15 CFR (Code of Federal Regulations).

NBS Interagency Reports (NBSIR)_A special series of interim or final reports on work performed by NBS for outside sponsors (both government and non-government). In general, initial distribution is handled by the sponsor; public distribution is by the National Technical Information Services (Springfield, Va. 22161) in paper copy or microfiche form.
Superconducting Devices and Materials. A literature survey issued quarterly. Annual subscription: $\$ 30.00$. Send subscription orders and remittances for the preceding bibliographic services to National Bureau of Standards, Cryogenic Data Center (275.02) Boulder, Colorado 80302. 
POSTAGE AND FEES PAID U.S. OEPARTMENT OF COMMERCE COM-215 